\title{
CLASSIFICATION OF THREE-DIMENSIONAL EXCEPTIONAL LOG CANONICAL HYPERSURFACE SINGULARITIES II
}

\author{
S. A. KUDRYAVTSEV
}

\begin{abstract}
In this paper the detailed classification of threedimensional exceptional canonical hypersurface singularities which don't satisfy the condition of well-formedness is given. This result completes the classification of three-dimensional exceptional log

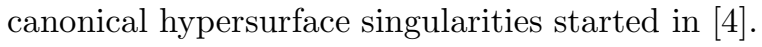

\section{Introduction}

In this paper the classification of three-dimensional exceptional hypersurface singularities started in [4] is completed. The main classification theorem is the following one:

Main theorem. Let $(X, 0) \subset\left(\mathbb{C}^{4}, 0\right)$ be a three-dimensional exceptional canonical (respectively strictly log canonical) hypersurface singularity defined by a polynomial $f$. Then there exists a biholomorphic coordinate change $\psi:\left(\mathbb{C}^{4}, 0\right) \rightarrow\left(\mathbb{C}_{t, z, x, y}^{4}, 0\right)$ and unique primitive vector $\mathbf{p} \in N_{\mathbb{R}}$ such that just one of the following two possibilities holds:

1. The quasihomogeneous polynomial $\tilde{f}_{\mathbf{p}}=(f \circ \psi)_{\mathbf{p}}$ defines an exceptional canonical (respectively strictly log canonical and canonical outside $\mathbf{0})$ singularity $\left(X_{\mathbf{p}}, 0\right) \subset\left(\mathbb{C}_{t, z, x, y}^{4}, 0\right)$. In this case $\mathbf{p}$-blow-up of $\mathbb{C}^{4}$ induces purely log terminal blow-ups $\varphi:(Y, E) \rightarrow(X, 0)$ and $\varphi_{\mathbf{p}}:\left(Y_{\mathbf{p}}, E_{\mathbf{p}}\right) \rightarrow\left(X_{\mathbf{p}}, 0\right)$, where $\left(E, \operatorname{Diff}_{E}(0)\right)=\left(E_{\mathbf{p}}, \operatorname{Diff}_{E_{\mathbf{p}}}(0)\right)$. That is, these singularities have the same type and in particular the same complement index.

The canonical singularities satisfying the condition of wellformedness - $\operatorname{Diff}_{E / \mathbb{P}(\mathbf{p})}(0)=0$ are classified in the theorems 3.24, 3.28 of [4] and in the tables of chapter [4, §4]. The polynomial $\tilde{f}_{\mathbf{p}} ;\left(E, \operatorname{Diff}_{E}(0)\right)$; minimal complement index are written in the tables.

The canonical singularities not satisfying the condition of wellformedness are classified in the tables of chapter $\S 3$. The polynomial $\tilde{f}_{\mathbf{p}} ;\left(E, \operatorname{Diff}_{E}(0)\right)$; minimal complement index are written in the tables. 
The strictly log canonical and canonical outside $\mathbf{0}$ quasihomogeneous singularities are always exceptional (in any dimension) by theorem [1, 2.13]. In the three-dimensional case $\left(E, \operatorname{Diff}_{E}(0)\right)=$ $\left(\tilde{f}_{\mathbf{p}} \subset \mathbb{P}(\mathbf{p}), 0\right)$ is $K 3$ surface with Du Val singularities and $(X, 0)$ is 1-complementary.

2. $\tilde{f}_{\mathbf{p}}=t^{3}+g_{2}^{2}(z, x, y)$, where $g_{2}$ is an irreducible homogeneous polynomial of degree two. In this case the purely log terminal blow-ups are constructed in the theorems $\mathbb{4}, 3.3,3.5]$. Also it was obtained the similar classification depending on the type of jets $\tilde{f}_{5}$ and $\tilde{f}_{6}$.

Proof. According to the main theorem proved in [4 it is enough to classify the three-dimensional exceptional canonical quasihomogeneous singularities which are not well-formed. The required classification is given in the tables of chapter $\S 3$.

It follows from the classification that there are only finite number of types of three-dimensional exceptional log canonical hypersurface singularities. There is a conjecture that the similar result about the finite number of types is true in any dimension for the exceptional singularities. See also examples «, 1.19, 1.20].

The present paper is the direct continuation of [4]. Therefore see [4] in connection with the notations, definitions and etc.

The paper is organized in the following way. In chapter $\S 1$ the purely log terminal blow-ups of not well-formed singularities are studied. In chapter $\S 2$ the three-dimensional quasihomogeneous not well-formed singularities are considered. In chapter $\S 3$ the summarizing tables are written.

I am grateful to Professor Yu.G.Prokhorov for useful discussions and valuable remarks.

The research was partially supported by a grant 99-01-01132 from the Russian Foundation of Basic Research and a grant INTAS-OPEN 2000\#269.

\section{Structure of purely log terminal blow-up of not well-formed singularity}

Now we explain in detail the condition of well-formedness of quasihomogeneous singularity.

Let $(X, 0) \subset\left(\mathbb{C}^{n}, 0\right)$ be a quasihomogeneous log canonical, canonical outside $\mathbf{0}$ singularity with the integer weights $\mathbf{p}=\left(p_{1}, \ldots, p_{n}\right)$ and quasihomogeneous degree $d$ defined by a polynomial $g\left(x_{1}, \ldots, x_{n}\right)$. Without loss of generality it can be assumed that $\left(p_{1}, \ldots, p_{n}\right)=1$.

Consider p-blow-up $\psi: \mathbb{C}^{n}(\mathbf{p}) \rightarrow \mathbb{C}^{n}$. The exceptional divisor of $\psi$ is the weighted projective space $\mathbb{P}(\mathbf{p})$. This blow-up induces a 
plt blow-up $\left.\psi\right|_{X \mathbf{p}}:(X(\mathbf{p}), E) \rightarrow(X, 0)$ (see theorem 2.13 [4]). Put $q_{i}=\left(p_{1}, \ldots, \hat{p}_{i}, \ldots, p_{n}\right)$. Then $E=\left(\tilde{g}\left(x_{1}, \ldots, x_{n}\right) \subset \mathbb{P}\left(\tilde{p}_{1}, \ldots, \tilde{p}_{n}\right)\right)=$ $\left(g\left(x_{1}^{1 / q_{1}}, \ldots, x_{n}^{1 / q_{n}}\right) \subset \mathbb{P}\left(p_{1} q_{1} /\left(q_{1} \cdots q_{n}\right), \ldots, p_{n} q_{n} /\left(q_{1} \cdots q_{n}\right)\right)\right.$. Put $q_{i j}=\left(\tilde{p}_{1}, \ldots, \widehat{\tilde{p}}_{i}, \ldots, \widehat{\tilde{p}}_{j}, \ldots, \tilde{p}_{n}\right)$ if $i \neq j$. The quasihomogeneous degree of $\tilde{g}\left(x_{1}, \ldots, x_{n}\right)$ is denoted by $\tilde{d}$.

Definition 1.1. [1] In the above-mentioned notations the quasihomogeneous singularity is called well-formed singularity if one of the following equivalent conditions holds:

1. $\operatorname{Diff}_{E / \mathbb{P}(\mathbf{p})}(0)=0$;

2. One have $q_{i j} \mid \tilde{d}$ for all $i \neq j$.

In opposite case our singularity is called not well-formed singularity. Let us remark that if we choose the other quasihomogeneous weights then it can happen that the condition of well-formedness is not fulfilled. For the weakly exceptional and consequently for the exceptional

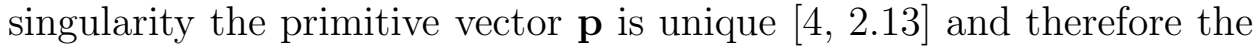
notion of well-formedness is uniquely defined.

Put $I_{i}=\left\{j \mid 1 \leq j \leq n, j \neq i, q_{i j} \nmid \tilde{d}\right\}$.

\section{Proposition 1.2.}

$$
\operatorname{Diff}_{E}(0)=\sum_{i=1}^{n}\left(1-\frac{1}{q_{i}}\right)\left(C_{i} \backslash \bigcup_{j \in I_{i}} C_{i j}\right)+\sum_{i<j ; j \in I_{i}}\left(1-\frac{1}{q_{i j} \cdot q_{i} \cdot q_{j}}\right) C_{i j},
$$

where the divisors $C_{i}$ and $C_{i j}$ on $E$ are defined by the equations $x_{i}=0$ and $x_{i}=x_{j}=0$ respectively. Moreover $C_{i}^{\prime} \stackrel{\text { def }}{=} C_{i} \backslash \bigcup_{j \in I_{i}} C_{i j}=\left\{x_{i}=\right.$ $\left.\left(\prod_{j \in I_{i}} x_{j}\right)^{-1} \cdot g\left(x_{1}, \ldots, x_{i-1}, 0, x_{i+1}, \ldots, x_{n}\right)=0\right\}$, where $q_{i} \neq 1$.

Proof. To calculate the different we have to determine the codimension 2 components of Sing $X(\mathbf{p})$ in $X(\mathbf{p})$ which are lying in $E$. The variety $X(\mathbf{p})$ in the affine piece $U_{k}=$ $\mathbb{C}_{x_{1}, \ldots, x_{n}}^{n} / \mathbb{Z}_{p_{k}}\left(-p_{1}, \ldots,-p_{k-1}, 1,-p_{k+1}, \ldots,-p_{n}\right)$ of $\mathbf{p}$-blow-up is given by a polynomial $g^{\prime}=g\left(x_{1}, \ldots, x_{k-1}, 1, x_{k+1}, \ldots, x_{n}\right)=0$. It is clear that the singularity defined by this polynomial $g^{\prime}$ has the codimension not less then 3 in $\mathbb{C}^{n-1} \cong\left\{x_{k}=0\right\}$ and hence doesn't influence on $\operatorname{Diff}_{E}(0)$ (recall that $E \bigcap U_{k}$ is given by a polynomial $g^{\prime}$ in $\left.\left\{x_{k}=0\right\} / \mathbb{Z}_{p_{k}}\right)$. Thus Diff $E_{E}(0)$ appears only because of the action of cyclic group. Since $q_{i j} \cdot q_{i} \cdot q_{j}=\left(p_{1}, \ldots, \hat{p}_{i}, \ldots, \hat{p}_{j}, \ldots, p_{n}\right)$ then it remains to show the following statement: if $q_{i j} \nmid \tilde{d}$ then $\left\{x_{i}=x_{j}=0\right\}$ defines a divisor on $E$. Actually, this condition means that $x_{i}$ or $x_{j}$ divides any monomial of $g$. 
Remark 1.3. If $q_{i j} \nmid \tilde{d}$ then $g=x_{i} g_{1}\left(x_{1}, \ldots, x_{n}\right)+x_{j} g_{2}\left(x_{1}, \ldots, x_{n}\right)$. Moreover, if $q_{i}>1$ and $q_{i j} \nmid \tilde{d}$ then $q_{j}=1$. Indeed, if $q_{j}>1$ then $g=x_{i}^{q_{i}} g_{1}\left(x_{1}, \ldots, x_{n}\right)+x_{j}^{q_{j}} g_{2}\left(x_{1}, \ldots, x_{n}\right)$. Hence $g$ defines a nonnormal singularity.

The next proposition is proved in the same way as the previous proposition 1.2 .

\section{Proposition 1.4.}

$$
\operatorname{Diff}_{E / \mathbb{P}(\mathbf{p})}(0)=\sum_{i<j ; j \in I_{i}}\left(1-\frac{1}{q_{i j}}\right) C_{i j} .
$$

Corollary 1.5. (cf. [2, 2.10]) Let $(X, 0) \subset\left(\mathbb{C}^{n}, 0\right)$ be a quasihomogeneous strictly log canonical, canonical outside $\mathbf{0}$ singularity with weight p. Then it is well-formed (by theorem 2.13 国 it is exceptional).

Proof. Let $\psi:(X(\mathbf{p}), E) \rightarrow(X, 0)$ be a plt blow-up. Since $K_{X(\mathbf{p})}+E=$ $\psi^{*} K_{X}$ is Cartier divisor then $\operatorname{Diff}_{E}(0)=0$. Hence by propositions 1.2 and $1.4 \operatorname{Diff}_{E / \mathbb{P}(\mathbf{p})}(0)=0$. In particular $E$ is $K 3$ surface with Du Val singularities if $n=4$ [1, 1.4.1].

Example 1.6. Let $(X, 0) \subset\left(\mathbb{C}^{3}, 0\right)$ be a weakly exceptional Du Val singularity defined by a polynomial $h$. Then $h$ is one of the following polynomials: $x_{1}^{2}+x_{2}^{2} x_{3}+x_{3}^{n-1}, n \geq 4$ (type $\mathbb{D}_{n}$ ); $x_{1}^{2}+x_{2}^{3}+x_{3}^{4}$ (type $\mathbb{E}_{6}$ ); $x_{1}^{2}+x_{2}^{3}+x_{3}^{3} x_{2}$ (type $\mathbb{E}_{7}$ ); $x_{1}^{2}+x_{2}^{3}+x_{3}^{5}$ (type $\mathbb{E}_{8}$ ) (see [0, 4.7]). Consider p-blow-ups corresponding to their quasihomogeneous weights. Then the singularities $\mathbb{D}_{n}, n \geq 5$ and $\mathbb{E}_{7}$ are not well-formed.

\begin{tabular}{|c|c|c|c|}
\hline Singularity & $E \cong \mathbb{P}^{1}$ & $\operatorname{Diff}_{E / \mathbb{P}(\mathbf{p})}(0)$ & $\operatorname{Diff}_{E}(0)$ \\
\hline$D_{2 n}, n \geq 3$ & $x_{1}+x_{2}^{2} x_{3}+x_{3}^{2 n-1} \subset$ & $\frac{n-2}{n-1} P_{3}$ & $\frac{1}{2} P_{1}+\frac{1}{2} P_{2}+$ \\
& $\subset \mathbb{P}(2 n-1, n-1,1)$ & & $+\frac{2 n-3}{2 n-2} P_{3}$ \\
\hline$D_{2 n+1}, n \geq 2$ & $x_{1}^{2}+x_{2} x_{3}+x_{3}^{2 n} \subset$ & $\frac{2 n-2}{2 n-1} P_{3}$ & $\frac{1}{2} P_{1}+\frac{1}{2} P_{2}+$ \\
& $\subset \mathbb{P}(n, 2 n-1,1)$ & & $+\frac{2 n-2}{2 n-1} P_{3}$ \\
\hline$E_{7}$ & $x_{1}+x_{2}^{3}+x_{3} x_{2} \subset$ & $\frac{1}{2} P_{3}$ & $\frac{1}{2} P_{1}+\frac{2}{3} P_{2}+$ \\
& $\subset \mathbb{P}(3,1,2)$ & & $+\frac{3}{4} P_{3}$ \\
\hline
\end{tabular}

Definition 1.7. Let us define a $\mathbb{Q}$-divisor $\widehat{D}=\sum_{i=1}^{n}\left(1-\frac{1}{q_{i}}\right)\left\{x_{i}=0\right\}$ on $\mathbb{P}(\mathbf{p})$ and a $\mathbb{Q}$-divisor $D=\sum_{i=1}^{n}\left(1-\frac{1}{q_{i}}\right) C_{i}$ on $E$. Recall that, if $(X, 0)$ is a well-formed singularity then $D=\left.\widehat{D}\right|_{E}=\operatorname{Diff}_{E}(0)$ (cf. 四, 
3.12]). Nevertheless the following proposition takes place in the general case.

Proposition 1.8. $K_{E}+\operatorname{Diff}_{E}(0)=\left.\left(K_{\mathbb{P}(\mathbf{p})}+E+\widehat{D}\right)\right|_{E}$.

Proof. By proposition 1.4, remark 1.3 and corollary [6, 3.10] $\left(K_{\mathbb{P}(\mathbf{p})}+\right.$ $E+\widehat{D})\left.\right|_{E}=K_{E}+\operatorname{Diff}_{E / \mathbb{P}(\mathbf{p})}(\widehat{D})=K_{E}+\sum_{i<j ; j \in I_{i}}\left[\left(1-\frac{1}{q_{i j}}\right) C_{i j}+\frac{1}{q_{i j}}\right.$. $\left.\left(1-\frac{1}{q_{i} \cdot q_{j}}\right) C_{i j}\right]+\sum_{i=1}^{n}\left(1-\frac{1}{q_{i}}\right)\left(C_{i} \backslash \bigcup_{j \in I_{i}} C_{i j}\right)$. By proposition 1.2 the last expression is equal to $K_{E}+\operatorname{Diff}_{E}(0)$.

Example 1.9. Let us return to the example 1.6. Then

\begin{tabular}{|c|c|c|}
\hline Singularity & $D$ & $\operatorname{Diff}_{E}(0)=\operatorname{Diff}_{E / \mathbb{P}(\mathbf{p})}(\widehat{D})$ \\
\hline$D_{2 n}, n \geq 3$ & $\frac{1}{2} P_{1}+\frac{1}{2} P_{2}+\frac{1}{2} P_{3}$ & $\begin{array}{c}\frac{1}{2} P_{1}+\frac{1}{2} P_{2}+ \\
+\left(\frac{n-2}{n-1}+\frac{1}{n-1} \cdot \frac{1}{2}\right) P_{3}\end{array}$ \\
\hline$D_{2 n+1}, n \geq 2$ & $\frac{1}{2} P_{1}+\frac{1}{2} P_{2}$ & $\begin{array}{c}\frac{1}{2} P_{1}+\frac{1}{2} P_{2}+ \\
+\left(\frac{2 n-2}{2 n-1}+\frac{1}{2 n-1} \cdot 0\right) P_{3}\end{array}$ \\
\hline$E_{7}$ & $\frac{1}{2} P_{1}+\frac{2}{3} P_{2}+\frac{1}{2} P_{3}$ & $\begin{array}{c}\frac{1}{2} P_{1}+\frac{2}{3} P_{2}+ \\
+\left(\frac{1}{2}+\frac{1}{2} \cdot \frac{1}{2}\right) P_{3}\end{array}$ \\
\hline
\end{tabular}

\section{Investigation of three-dimensional not well-formed singularities on exceptionality}

The next theorem is proved by exhaustion of all cases. This sorting is described in 体.

Theorem 2.1. All three-dimensional not well-formed exceptional canonical quasihomogeneous singularities can be obtained by the rotations of Newton's line passing through the following parts of type $\mathcal{M}_{2}$ :

(1) $t^{2}+z^{3}+x^{m}$, where $x^{m}-z x^{5}, z x^{5} y, z x^{7}, z x^{6} y, z x^{5} y^{2}$.

(2) $t^{2}+z^{4}+x^{m}$, where $x^{m}-z x^{4}, z x^{5}, z x^{4} y$.

(3) all parts of type $\Upsilon_{2}^{(2)}$ (they have the equation of the form $t^{2}+$ $\left.z^{3} x+h(z, x, y)\right)$.

(4) $t^{2}+z^{3} y+x^{m}$, where $x^{m}-x^{7} y, x^{8} y, z x^{5}, z x^{6}, z x^{5} y$.

(5) $t^{2}+z^{5}+z x^{5} ; t^{2}+z^{4} x+x^{m}$, where $x^{m}-x^{6}, x^{5} y, x^{4} y^{2}, z x^{5}$, $z x^{4} y, z x^{3} y^{2} ; t^{2}+z^{4} y+x^{m}$, where $x^{m}-x^{5} y, z x^{5}, z x^{4} y ; t^{2}+z^{3} x^{2}+x^{m}$, where $x^{m}-z x^{5}, z x^{4} y, z x^{3} y^{2} ; t^{2}+z^{3} y^{2}+z x^{5} ; t^{2}+z^{3} x y+x^{m}$, where $x^{m}-x^{6}, x^{5} y, x^{7}, x^{6} y, x^{5} y^{2}, z x^{5}, z x^{4} y ; t^{2}+z^{5}+z x^{3} y ; t^{2}+z^{4} x+z x^{3} y$; $t^{2}+z^{4} x+x^{4} y ; t^{2}+z^{3} x^{2}+z x^{3} y$. 
(6) $t^{3}+z^{2} x y+x^{5} ; t^{3}+z^{2} x y+x^{4} y ; t^{3}+z^{4}+z x^{2} y ; t^{3}+z^{4}+t x^{3}$; $t^{3}+z^{3} x+x^{m}$, where $x^{m}-z x^{2} y, x^{3} y, t x^{3}, t x^{2} y ; t^{3}+z^{3} y+t x^{3} ; t^{3}+t z^{3}+$ $x^{m}$, where $x^{m}-z^{2} x^{2}, z x^{3}, z x^{2} y ; t^{3}+t z^{2} x+z x^{2} y ; t^{3}+t z^{2} x+x^{2} y^{2}$; $g_{3}(t, z)+z x^{3}$, where $g_{3}$ is a binary form of degree $3 ; t^{3}+z^{2} x+x^{m}$, where $x^{m}-x^{4}, x^{5}, x^{4} y, x^{6}+t x^{4}, x^{5} y, x^{4} y^{2}, x^{7}, x^{6} y, x^{5} y^{2}, x^{4} y^{3}$, $x^{8}, x^{7} y, x^{6} y^{2}, x^{5} y^{3}, x^{4} y^{4}, t x^{3}, t x^{3} y, t x^{5}, t x^{4} y, t x^{3} y^{2} ; t^{3}+z^{2} y+x^{7} y$; $t^{3}+z^{2} y+t x^{5}$.

(7) $t^{2} z+g_{4}(z, x)$, where $g_{4}$ is a binary form of degree $4 ; t^{2} z+z^{4}+x^{m}$, where $x^{m}-x^{3} y, x^{5}, x^{4} y, x^{3} y^{2}, t x^{3}, t x^{2} y, t^{2} z+z^{3} x+x^{m}$, where $x^{m}$ - $x^{3} y, x^{5}, x^{4} y, x^{3} y^{2}, t x^{3}, t x^{2} y ; t^{2} z+z^{3} y+x^{m}$, where $x^{m}-x^{5}$, $x^{4} y, z x^{3}, z^{2} x^{2}, t x^{3} ; t^{2} z+z^{2} x^{2}+x^{m}$, where $x^{m}-x^{5}, x^{4} y, x^{3} y^{2}, t x^{2} y$; $t^{2} z+z^{2} x y+x^{5} ; t^{2} z+z^{2} x y+x^{4} y ; t^{2} z+z^{2} x+x^{m}$, where $x^{m}-x^{4}$, $z x^{3}+x^{5}, x^{4} y, x^{6}, x^{5} y, x^{4} y^{2}, t x^{3}, t x^{4}, t x^{3} y ; t^{2} z+z^{2} y+x^{m}$, where $x^{m}$ $-x^{5} y, z x^{3}, t x^{4}$.

(8) $t^{2} x+z^{4}+x^{m}$, where $x^{m}-x^{3} y, x^{5}, x^{4} y, z x^{2} y, z x^{4}, z x^{3} y, z x^{2} y^{2}$; $t^{2} x+z^{3} x+x^{m}$, where $x^{m}-x^{2} y^{2}, x^{5}, x^{4} y, x^{3} y^{2}, x^{2} y^{3}, x^{6}, x^{5} y, x^{4} y^{2}$, $x^{3} y^{3}, x^{2} y^{4}, z x^{2} y, z x^{4}, z x^{3} y, z x^{2} y^{2} ; t^{2} x+z^{3} y+x^{m}$, where $x^{m}-x^{5}$, $x^{4} y, x^{6}, x^{5} y, x^{4} y^{2}, z x^{4}, z x^{3} y, z^{2} x^{2}$.

(9) $t^{2} y+z^{4}+z x^{4} ; t^{2} y+z^{3} x+x^{m}$, where $x^{m}-x^{5}, x^{6}, x^{5} y, z x^{4}, t x^{3}$.

In investigating given singularities on the exceptionality it is convenient to use the proposition [ $4,3.13]$ and corollary $[4,3.14]$. In general case corollary $₫, 3.15]$ is formulated in the following way.

Proposition 2.2. Let $(X, 0) \subset\left(\mathbb{C}_{x_{1}, \ldots, x_{4}}^{4}, 0\right)$ be a three-dimensional quasihomogeneous log canonical singularity with the weight $\mathbf{p}$. Assume that $(X, H)$ is not lc for any hyperplane section $H$. Let $D=\sum d_{i} D_{i}$ (see definition 1.7). If $d_{k} \geq \frac{6}{7}$ for some $k$ then $(X, 0)$ is exceptional.

Proof. Suppose that $(X, 0)$ is not exceptional. Let $H_{X}=\left.\left\{x_{k}=0\right\}\right|_{X}$ be a hyperplane section and let $H_{X(\mathbf{p})}$ be a proper transform of $H_{X}$ on $X(\mathbf{p})$. Then $K_{X(\mathbf{p})}+E+H_{X(\mathbf{p})}=\psi^{*}\left(K_{X}+H_{X}\right)+a E$. Let $C_{k}=\sum_{i} C_{k i}$, where $C_{k i}$ are the irreducible curves (see proposition 1.2). One have $\operatorname{Diff}_{E}(0)=\sum_{i} d_{k i} C_{k i}+\Xi$. The coefficients $d_{k i} \geq 6 / 7$ for all $i$ since $d_{k i}=\frac{m_{i}-1}{m_{i}}+\frac{1}{m_{i}} \cdot d_{k} \geq d_{k}$ [6, 3.10]. By proposition 田, 3.14] there exists 1,2,3,4 or 6-complement $D^{+}$. By the definition of complement we have $D^{+} \geq \sum_{i} C_{k i}+\Xi$. Therefore $-\left(K_{E}+\sum_{i} C_{k i}+\Xi\right)$ is nef and lc. Since $\operatorname{Diff}_{E}\left(H_{X(\mathbf{p})}\right)=\operatorname{Diff}_{E / \mathbb{P}(\mathbf{p})}\left(\widehat{D}+\left\{x_{k}=0\right\}\right)$, where $\left\{x_{k}=0\right\}$ is the corresponding divisor on $\mathbb{P}(\mathbf{p})$ then $\operatorname{Diff}_{E}\left(H_{X(\mathbf{p})}\right)=\sum_{i} C_{k i}+\Xi$. Hence $a \geq 0$ and $\left(X, H_{X}\right)$ is lc. This contradiction concludes the proof.

Remark 2.3. Proposition 2.2 is hypothetically true in any dimension (see [ $\left[\right.$, 3.16]). In the two-dimensional case we must require that $d_{k} \geq \frac{2}{3}$ for some $k$. 
Example 2.4. In proposition 2.2 we can't change $D$ on $\operatorname{Diff}_{E}(0)$. Let us show it in the following two examples.

(1) Let $(X, 0) \subset\left(\mathbb{C}^{3}, 0\right)$ be a Du Val singularity of type $\mathbb{D}_{n}$ defined by a polynomial $x_{1}^{2}+x_{2}^{2} x_{3}+x_{3}^{n-1}$, where $n \geq 4$. The pair $(X, H)$ is not lc for any hyperplane section $H$. If $n \geq 5$ then one of the coefficients of $\operatorname{Diff}_{E}(0)$ is not less then $2 / 3$ and all coefficients of $D$ are equal to $1 / 2$. This singularity is not exceptional by the definition since $K_{X}+\left\{x_{1}=x_{3}=0\right\} \sim_{\mathbb{Q}} 0$ is 2-complement (see also example [4, 1.8]).

(2) Let $(X, 0) \subset\left(\mathbb{C}_{t, z, x, y}^{4}, 0\right)$ be a canonical singularity defined by a polynomial $t^{3}+z^{2} x+x^{4}+x y^{5}$. The pair $(X, H)$ is not lc for any hyperplane section $H$. The variety $X$ has $c A_{2}$ singularity along the curve $C=\left\{t=x=z^{2}+y^{5}=0\right\}=\operatorname{Sing} X$ (outside $\mathbf{0}$ ). The divisor $T=\{t=x=0\}$ on $X$ is $\mathbb{Q}$-Cartier since $3 T \sim\{x=0\}$. Therefore the divisor $\left.\left(K_{X}+T\right)\right|_{T}=K_{\mathbb{C}_{z, y}^{2}}+\frac{2}{3} C$ is klt. Thus $(X, 0)$ is not weakly exceptional singularity [5, 4.6].

Now consider $(40,45,30,18)$-blow-up of $\mathbb{C}^{4}$. It induces a plt blow-up of $(X, 0)$. Then $\left(E, \operatorname{Diff}_{E}(0)\right)=\left(t+z x+x^{4}+x y \subset \mathbb{P}(4,3,1,3), \frac{2}{3}(\{t=\right.$ $\left.0\} \backslash\{t=x=0\})+\frac{1}{2}\{z=0\}+\frac{4}{5}\{y=0\}+\frac{8}{9}\{t=x=0\}\right)=\left(\mathbb{P}^{2}, \frac{2}{3} l_{1}+\right.$ $\left.\frac{1}{2} l_{2}+\frac{4}{5} l_{3}+\frac{8}{9} l_{4}\right)$, where $l_{i}$ are the straight lines in general position. The divisor $K_{\mathbb{P}^{2}}+\frac{2}{3} l_{1}+\frac{1}{2} l_{2}+\frac{5}{6} l_{3}+l_{4} \sim_{\mathbb{Q}} 0$ is klt 6 -complement of minimal index. Since the divisor $K_{\mathbb{P}^{2}}+\operatorname{Diff}_{E}(0)+\frac{1}{9} l_{4}$ is anti-ample and non-klt then by criterion [3, 2.1] $(X, 0)$ is not weakly exceptional singularity again. In this example all coefficients of $D$ are not more then $\frac{4}{5}$.

It is very interesting to compare the following example 2.5 with the previous example 2.4 (2).

Example 2.5. Let $(X, 0) \subset\left(\mathbb{C}_{t, z, x, y}^{4}, 0\right)$ be a canonical singularity defined by a polynomial $t^{3}+z^{2} x+t x^{3}+t y^{5}$. The variety $X$ has $c A_{1}$ singularity along the curve $C=\left\{t=z=x^{3}+y^{5}=0\right\}=\operatorname{Sing} X$ (outside 0). Consider the divisor $T=\{t=z=0\}$ on $X$. If $T$ had been $\mathbb{Q}$-Cartier divisor then the divisor $\left.\left(K_{X}+T\right)\right|_{T}=K_{\mathbb{C}_{x, y}^{2}}+\frac{1}{2} C$ would have been klt and consequently $(X, 0)$ would have been not weakly exceptional singularity. Now we prove that $T$ is not $\mathbb{Q}$-Cartier divisor and $(X, 0)$ is an exceptional singularity.

Consider $\mathbf{p}=(30,35,20,12)$-blow-up of $\mathbb{C}^{4}$. It induces a plt blow-up $\psi:(X(\mathbf{p}), E) \rightarrow(X, 0)$. Then $E=\left(t^{3}+z x+t x^{3}+t y \subset \mathbb{P}(3,7,2,6)\right)$, $\operatorname{Diff}_{E}(0)=\frac{1}{2}\left\{z=t^{2}+x^{3}+y=0\right\}+\frac{3}{4}\{t=z=0\}+\frac{4}{5}\{y=0\}=$ $\frac{1}{2} \Delta+\frac{3}{4} \Gamma_{2}+\frac{4}{5} L$. In the next picture we illustrate the situation of these curves and the singularities of surface $E$. 


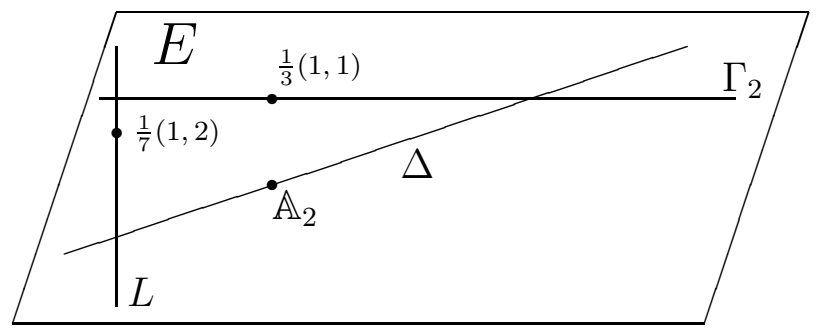

Assume that $T$ is $\mathbb{Q}$-Cartier divisor. Then $K_{X(\mathbf{p})}+E+T_{Y}=$ $\psi^{*}\left(K_{X}+T\right)+a E$ is anti-ample divisor over $X$ since $a>0$. Hence $K_{E}+\operatorname{Diff}_{E}\left(T_{Y}\right)=K_{E}+\frac{1}{2} \Delta+\Gamma_{2}+\frac{4}{5} L$ is anti-ample divisor. It is not true since $\left(K_{E}+\operatorname{Diff}_{E}\left(T_{Y}\right)\right) \cdot \Delta=\left(K_{E}+\frac{1}{2} \Delta+\frac{3}{4} \Gamma_{2}+\frac{11}{12} L\right) \cdot \Delta+\left(\frac{1}{4} \Gamma_{2}+\left(\frac{4}{5}-\right.\right.$ $\left.\left.\frac{11}{12}\right) L\right) \cdot \Delta=\left(K_{\mathbb{P}(\mathbf{p})}+E+\frac{1}{2}\{z=0\}+\frac{11}{12}\{y=0\}\right) \cdot \Delta+\frac{1}{4}-\frac{7}{60}=0+\frac{1}{4}-\frac{7}{60}>0$. Here $\{z=0\}$ and $\{y=0\}$ are denoted the corresponding divisors on $\mathbb{P}(\mathbf{p})$.

The divisor $K_{E}+\operatorname{Diff}_{E / \mathbb{P}(\mathbf{p})}\left(\frac{3}{5}\{z=0\}+\frac{4}{5}\{y=0\}\right)=K_{E}+\frac{3}{5} \Delta+\frac{4}{5} \Gamma_{2}+$ $\frac{4}{5} L$ is 5 -complement of minimal index. It is easy to show that there is only the following 6-complement $K_{E}+\operatorname{Diff}_{E / \mathbb{P}(\mathbf{p})}\left(\frac{1}{6}\{t=0\}+\frac{1}{2}\{z=\right.$ $\left.0\}+\frac{5}{6}\{y=0\}\right)=K_{E}+\frac{1}{2} \Delta+\frac{5}{6} \Gamma_{2}+\frac{5}{6} L+\frac{1}{6}\{t=x=0\} \sim_{\mathbb{Q}} 0$ among $1,2,3,4$ and 6 -complements. It is clear that this divisor is klt. Therefore $(X, 0)$ is an exceptional singularity.

Finally let us consider one more example showing the investigation process of given singularities on the exceptionality.

Example 2.6. Let $(X, 0) \subset\left(\mathbb{C}_{t, z, x, y}^{4}, 0\right)$ be a canonical singularity defined by a polynomial $f=t^{2} x+z^{3} x+z x^{2} y+a t z^{2} y+b z^{2} y^{3}+c x y^{4}$. Consider $\mathbf{p}=(6,4,5,3)$-blow-up of $\mathbb{C}^{4}$. Then $\left(E, \operatorname{Diff}_{E}(0)\right)=(f \subset$ $\left.\mathbb{P}(6,4,5,3), \frac{2}{3} \Delta_{3}+\frac{1}{2} \Upsilon_{4}\right)$, where $\Delta_{3}=\{z=x=0\}$ and $\Upsilon_{4}=\{x=$ $y=0\}$. Let us prove that the divisor $\left.\left(K_{\mathbb{P}(\mathbf{p})}+E+\frac{1}{4}\{z=0\}\right)\right|_{E}=$ $K_{E}+\frac{3}{4} \Delta_{3}+\frac{1}{2} \Upsilon_{4}+\frac{1}{2}\{t=z=0\}$ is non-klt 4-complement if $c=0$ (then $b \neq 0)$. It implies that $(X, 0)$ is not exceptional singularity if $c=0$. Since the klt and lc properties of pair remain true by the finite dominant morphisms then it is enough to prove that $\left(Z, \frac{1}{4} T\right)$ is lc, but not klt pair, where $Z=\left\{t^{2} x+z^{3} x+z x^{2}+a t z^{2}+z^{2}=0\right\} \subset \mathbb{C}_{t, z, x}^{3}$ and $T=\left.\{z=0\}\right|_{Z}$. Note that $(Z, 0)$ is the singularity of type $\mathbb{D}_{5}$. The blow-up of $\mathbb{C}^{3}$ with the weights $(3,4,2)$ induces a blow-up $\psi:(\widetilde{Z}, C) \rightarrow Z$. Besides $K_{\widetilde{Z}}+C+\frac{1}{4} T_{\widetilde{Z}}=\psi^{*}\left(K_{Z}+\frac{1}{4} T\right)$. The exceptional divisor $C$ is not well-formed hypersurface $t x+z x^{2}+z^{2} \subset \mathbb{P}(3,2,1)$. The divisor $K_{C}+\operatorname{Diff}_{C}\left(\frac{1}{4} T_{\widetilde{Z}}\right)=K_{C}+\frac{1}{2} P_{1}+\left(\frac{1}{2}+\frac{1}{2} \cdot \frac{1}{2}\right) P_{2}+\left(\frac{2}{3}+\frac{1}{3} \cdot \frac{1}{4}\right) P_{3}$ is klt. By Inversion of Adjunction we complete the proof. If $c \neq 0$ then by the same argument one can prove that $(X, 0)$ is an exceptional singularity. 


\section{Classification of three-dimensional exceptional not well-formed singularities}

The comments to the tables. All agreements of $\S 4$ [4] about the notations and records in the tables remain valid. The underlying monomials (see point (I) of $\S 4$ 近) are written without the coefficients before them.

If $\log$ Del Pezzo surface $E=\left(\widetilde{f} \subset \mathbb{P}\left(\widetilde{p}_{1}, \ldots, \widetilde{p}_{4}\right)\right.$ is a linear cone, i.e. $\widetilde{d}=\widetilde{p}_{k}$ for some $k$ then we write the simplified form of $E$

$$
E=\mathbb{P}\left(\frac{\widetilde{p}_{1}}{s_{1}}, \ldots, \frac{\widetilde{p}_{k-1}}{s_{k-1}}, \frac{\widetilde{p}_{k+1}}{s_{k+1}}, \ldots, \frac{\widetilde{p}_{4}}{s_{4}}\right) \text {, where } s_{m}=\prod_{\substack{q_{i j} \tau \widetilde{d} \\ i<j ; j, j \neq m}} q_{i j}
$$

For the record of different $\operatorname{Diff}_{E}(0)$ one use the following notations. The curves $C_{i}^{\prime}$, where $i=1, \ldots, 4$ (see proposition 1.2) are denoted by $\Gamma, \Delta, \Upsilon, \Omega$ respectively. The curves $\{t=z=0\},\{t=x=0\}$, $\{t=y=0\},\{z=x=0\},\{z=y=0\},\{x=y=0\}$ are denoted by $\Gamma_{2}, \Gamma_{3}, \Gamma_{4}, \Delta_{3}, \Delta_{4}, \Upsilon_{4}$ respectively.

If there is a symbol * in the description of different cases then this marked case has the other different. Then the different differs by the addition of other curve marked by * or the different is completely another one marked by * also. 
1. Singularity $-t^{2}+z^{3}+g(z, x, y)$

\begin{tabular}{|c|c|c|c|}
\hline No. & $g(z, x, y)$ & Log Del Pezzo & Index \\
\hline 1 & $z x^{i} f_{5-i}\left(x, y^{3}\right)$ & $\begin{array}{l}t t+z^{3}+z x^{i} f_{5-i}\left(x, y^{3}\right) \subset \mathbb{P}(15,5,2,2) \\
\mathbb{P}(5,1,1), \text { Diff }=\left(0,0,0, \frac{2}{3}\right)+\frac{1}{2} \Gamma+\frac{3}{4} \Gamma_{2}\end{array}$ & 4 \\
\hline 2 & $\begin{array}{c}z x^{5}+z y^{n} \\
n=7 ; 9 ; 11 ; 13 ; 17,19\end{array}$ & $\begin{array}{c}t+z^{3}+z x+z y \subset \mathbb{P}(3,1,2,2) \\
\mathbb{P}^{2}, \text { Diff }=\left(0,0, \frac{4}{5}, \frac{n-1}{n}\right)+\frac{1}{2} \Gamma+\frac{3}{4} \Gamma_{2}\end{array}$ & $\begin{array}{c}8,10,12 \\
16,20\end{array}$ \\
\hline 3 & $\begin{aligned} & z x^{5}+z x y^{n} \\
n= & 5 ; 7 ; 9,11 ; 13,15\end{aligned}$ & $\begin{array}{c}t+z^{3}+z x^{5}+z x y \subset \mathbb{P}(15,5,2,8) \\
\mathbb{P}(5,1,4), \text { Diff }=\left(0,0,0, \frac{n-1}{2}\right)+\frac{1}{2} \Gamma+\frac{3}{4} \Gamma_{2}\end{array}$ & $\begin{array}{c}6,8,12 \\
16\end{array}$ \\
\hline 4 & $\begin{array}{c}z x^{5}+a z x^{3} y^{n}+b z x y^{2 n} \\
n=3 b \neq 0 ; 5,7\end{array}$ & $\begin{array}{l}t+z^{3}+z x^{5}+a z x^{3} y+b z x y^{2} \subset \mathbb{P}(15,5,2,4) \\
\mathbb{P}(5,1,2), \text { Diff }=\left(0,0,0, \frac{n-1}{n}\right)+\frac{1}{2} \Gamma+\frac{3}{4} \Gamma_{2}\end{array}$ & 4,8 \\
\hline 5 & $\begin{array}{c}z x^{5}+z x^{2} y^{n} \\
n=5,7 ; 11 \\
\end{array}$ & $\begin{array}{c}t+z^{3}+z x^{5}+z x^{2} y \subset \mathbb{P}(15,5,2,6) \\
\mathbb{P}(5,1,3), \text { Diff }=\left(0,0,0, \frac{n-1}{n}\right)+\frac{1}{2} \Gamma+\frac{3}{4} \Gamma_{2}\end{array}$ & 8,12 \\
\hline 6 & $\begin{array}{c}z x^{5} y+z y^{n} \\
n=7 ; 9 ; 13 ; 15\end{array}$ & $\begin{array}{c}t+z^{3}+z x y+z y^{n} \subset \mathbb{P}(3 n, n, 2 n-2,2) \\
\mathbb{P}(n, n-1,1), \text { Diff }=\left(0,0,0, \frac{n-1}{n}\right)+\frac{1}{2} \Gamma+\frac{3}{4} \Gamma_{2}\end{array}$ & $\begin{array}{c}8,12,16 \\
20\end{array}$ \\
\hline 7 & $z y x^{i} f_{5-i}\left(x, y^{2}\right)$ & $\begin{array}{c}t+z^{3}+z x^{i} f_{5-i}\left(x, y^{2}\right) \subset \mathbb{P}(33,11,4,2) \\
\mathbb{P}(11,2,1), \text { Diff }=\frac{1}{2} \Gamma+\frac{3}{4} \Gamma_{2}\end{array}$ & 4 \\
\hline 8 & $\begin{array}{c}z x^{5} y+z x y^{n} \\
n=6 ; 8,10 ; 12\end{array}$ & $\begin{array}{c}t+z^{3}+g(z, x, y) \subset \mathbb{P}(15 n-3,5 n-1,2 n-2,8) \\
\mathbb{P}(5 n-1, n-1,4), \text { Diff }=\frac{1}{2} \Gamma+\frac{3}{4} \Gamma_{2}\end{array}$ & $\overline{8,12,16}$ \\
\hline 9 & $\begin{array}{c}z x^{5} y+a z x^{3} y^{n+1}+b z x y^{2 n+1} \\
n=3 \quad b \neq 0 ; 5\end{array}$ & $\begin{array}{c}t+z^{3}+g(z, x, y) \subset \mathbb{P}(15 n+6,5 n+2,2 n, 4) \\
\mathbb{P}(5 n+2, n, 2), \text { Diff }=\frac{1}{2} \Gamma+\frac{3}{4} \Gamma_{2}\end{array}$ & 4,8 \\
\hline 10 & $\begin{array}{c}z x^{5} y+z x^{2} y^{n} \\
n=5 ; 9\end{array}$ & $\begin{array}{c}t+z^{3}+g(z, x, y) \subset \mathbb{P}(15 n-6,5 n-2,2 n-2,6) \\
\mathbb{P}(5 n-2, n-1,3), \text { Diff }=\frac{1}{2} \Gamma+\frac{3}{4} \Gamma_{2}\end{array}$ & 8,12 \\
\hline 11 & $\begin{array}{c}z x^{i} f_{7-i}(x, y) \\
i \leq 2\end{array}$ & $\begin{array}{c}t+z^{3}+z x^{i} f_{7-i}(x, y) \subset \mathbb{P}(21,7,2,2) \\
\mathbb{P}(7,1,1), \text { Diff }=\frac{1}{2} \Gamma+\frac{3}{4} \Gamma_{2}\end{array}$ & 4 \\
\hline 12 & $z x^{7}+z y^{9}$ & $\begin{array}{l}t+z^{3}+z x+z y \subset \mathbb{P}(3,1,2,2) \\
\mathbb{P}^{2}, \text { Diff }=\left(0,0, \frac{6}{7}, \frac{8}{9}\right)+\frac{1}{2} \Gamma+\frac{3}{4} \Gamma_{2}\end{array}$ & 28 \\
\hline 13 & $z x^{7}+z x y^{7}$ & $\begin{array}{l}t+z^{3}+z x^{7}+z x y \subset \mathbb{P}(21,7,2,12) \\
\mathbb{P}(7,1,6), \text { Diff }=\left(0,0,0, \frac{6}{7}\right)+\frac{1}{2} \Gamma+\frac{3}{4} \Gamma_{2}\end{array}$ & 8 \\
\hline 14 & $z x^{7}+z x^{3} y^{5}$ & $\begin{array}{c}t+z^{3}+z x^{7}+z x^{3} y \subset \mathbb{P}(21,7,2,8) \\
\mathbb{P}(7,1,4), \text { Diff }=\left(0,0,0, \frac{4}{5}\right)+\frac{1}{2} \Gamma+\frac{3}{4} \Gamma_{2}\end{array}$ & 16 \\
\hline 15 & $z x^{6} y+a z x^{3} y^{5}+b z y^{9}$ & $\begin{array}{c}t+z^{3}+z x^{2} y+a z x y^{5}+b z y^{9} \subset \mathbb{P}(27,9,8,2) \\
\mathbb{P}(9,4,1), \text { Diff }=\left(0,0, \frac{2}{3}, 0\right)+\frac{1}{2} \Gamma+\frac{3}{4} \Gamma_{2}\end{array}$ & 12 \\
\hline 16 & $\begin{array}{l}z x^{6} y+z x y^{n} \\
n=7 ; 8\end{array}$ & $\begin{array}{c}t+z^{3}+g(z, x, y) \subset \mathbb{P}(18 n-3,6 n-1,2 n-2,10) \\
\mathbb{P}(6 n-1, n-1,5), \text { Diff }=\frac{1}{2} \Gamma+\frac{3}{4} \Gamma_{2}\end{array}$ & 8,20 \\
\hline 17 & $\begin{array}{c}z x^{5} y^{2}+z y^{n} \\
n=9 ; 11\end{array}$ & $\begin{array}{l}t+z^{3}+z x y^{2}+z y^{n} \subset \mathbb{P}(3 n, n, 2 n-4,2) \\
\mathbb{P}(n, n-2,1), \text { Diff }=\left(0,0, \frac{4}{5}, 0\right)+\frac{1}{2} \Gamma+\frac{3}{4} \Gamma_{2}\end{array}$ & 16,20 \\
\hline 18 & $\begin{array}{c}z x^{5} y^{2}+z x y^{n} \\
n=7 ; 9\end{array}$ & $\begin{array}{c}t+z^{3}+g(z, x, y) \subset \mathbb{P}(15 n-6,5 n-2,2 n-4,8) \\
\mathbb{P}(5 n-2, n-2,4), \text { Diff }=\frac{1}{2} \Gamma+\frac{3}{4} \Gamma_{2}\end{array}$ & 12,16 \\
\hline 19 & $z x^{5} y^{2}+a z x^{3} y^{5}+b z x y^{8}$ & $\begin{array}{c}t+z^{3}+g(z, x, y) \subset \mathbb{P}(57,19,6,4) \\
\mathbb{P}(19,3,2), \text { Diff }=\frac{1}{2} \Gamma+\frac{3}{4} \Gamma_{2}\end{array}$ & 8 \\
\hline 20 & $z x^{5} y^{2}+z x^{2} y^{7}$ & $\begin{array}{c}t+z^{3}+g(z, x, y) \subset \mathbb{P}(93,31,10,6) \\
\mathbb{P}(31,5,3), \text { Diff }=\frac{1}{2} \Gamma+\frac{3}{4} \Gamma_{2}\end{array}$ & 12 \\
\hline
\end{tabular}

2. Singularity $-t^{2}+z^{4}+g(z, x, y)$ 


\begin{tabular}{|c|c|c|c|}
\hline 1 & $z x^{i} f_{4-i}\left(x, y^{2}\right)$ & $\begin{array}{c}t^{2}+z^{4}+z x^{i} f_{4-i}(x, y) \subset \mathbb{P}(8,4,3,3) \\
\text { Diff }=\left(0,0,0, \frac{1}{2}\right)+\frac{2}{3} \Gamma_{2}\end{array}$ & 3 \\
\hline 2 & $\begin{array}{l}z x^{4}+z y^{n} \\
n=5 ; 7 ; 11\end{array}$ & $\begin{array}{c}t^{2}+z^{4}+z x+z y \subset \mathbb{P}(2,1,3,3) \\
\text { Diff }=\left(0,0, \frac{3}{4}, \frac{n-1}{n}\right)+\frac{2}{3} \Gamma_{2}\end{array}$ & $5,8,12$ \\
\hline 3 & $z x^{4}+a z x^{2} y^{5}+b z y^{10}$ & $\begin{array}{c}t^{2}+z^{4}+z x^{2}+a z x y+b z y^{2} \subset \mathbb{P}(4,2,3,3) \\
\text { Diff }=\left(0,0, \frac{1}{2}, \frac{4}{5}\right)+\frac{2}{3} \Gamma_{2}\end{array}$ & 6 \\
\hline 4 & $\begin{array}{l}z x^{4}+z x y^{n} \\
n=4,5 ; 7,8\end{array}$ & $\begin{array}{c}t^{2}+z^{4}+z x^{4}+z x y \subset \mathbb{P}(8,4,3,9) \\
\text { Diff }=\left(0,0,0, \frac{n-1}{n}\right)+\frac{2}{3} \Gamma_{2}\end{array}$ & 5,9 \\
\hline 5 & $\begin{array}{l}z x^{i} f_{5-i}(x, y) \\
\quad i=1\end{array}$ & $\begin{array}{c}t^{2}+z^{4}+z x^{i} f_{5-i}(x, y) \subset \mathbb{P}(10,5,3,3) \\
\text { Diff }=\frac{2}{3} \Gamma_{2}\end{array}$ & 3 \\
\hline 6 & $z x^{5}+z y^{7}$ & $\begin{array}{c}t^{2}+z^{4}+z x+z y \subset \mathbb{P}(2,1,3,3) \\
\text { Diff }=\left(0,0, \frac{4}{5}, \frac{6}{7}\right)+\frac{2}{3} \Gamma_{2}\end{array}$ & 15 \\
\hline 7 & $z x^{5}+z x y^{5}$ & $\begin{array}{c}t^{2}+z^{4}+z x^{5}+z x y \subset \mathbb{P}(10,5,3,12) \\
\text { Diff }=\left(0,0,0, \frac{4}{5}\right)+\frac{2}{3} \Gamma_{2}\end{array}$ & 6 \\
\hline 8 & $z x^{5}+z x^{2} y^{4}$ & $\begin{array}{c}t^{2}+z^{4}+z x^{5}+z x^{2} y \subset \mathbb{P}(10,5,3,9) \\
\text { Diff }=\left(0,0,0, \frac{3}{4}\right)+\frac{2}{3} \Gamma_{2}\end{array}$ & 9 \\
\hline 9 & $z x^{4} y+a z x^{2} y^{4}+b z y^{7}$ & $\begin{array}{c}t^{2}+z^{4}+z x^{2} y+a z x y^{4}+b z y^{7} \subset \mathbb{P}(14,7,9,3) \\
\text { Diff }=\left(0,0, \frac{1}{2}, 0\right)+\frac{2}{3} \Gamma_{2}\end{array}$ & 6 \\
\hline 10 & $z x^{4} y+z y^{8}$ & $\begin{array}{c}t^{2}+z^{4}+z x y+z y^{8} \subset \mathbb{P}(16,8,21,3) \\
\text { Diff }=\left(0,0, \frac{3}{4}, 0\right)+\frac{2}{3} \Gamma_{2}\end{array}$ & 12 \\
\hline 11 & $\begin{array}{l}z x^{4} y+z x y^{n} \\
\quad n=5 ; 6\end{array}$ & $\begin{aligned} t^{2}+z^{4}+g(z, x, y) & \subset \mathbb{P}(8 n-2,4 n-1,3 n-3,9) \\
& \operatorname{Diff}=\frac{2}{3} \Gamma_{2}\end{aligned}$ & 6,9 \\
\hline
\end{tabular}

3. Singularity $-t^{2}+z^{3} x+g(z, x, y)$

\begin{tabular}{|c|c|c|c|}
\hline 1 & $\begin{array}{c}x^{5}+x y^{n} \\
n=7 ; 11 ; 13 ; 17 ; 19,23\end{array}$ & $\begin{array}{c}t+z x+x^{5}+x y \subset \mathbb{P}(5,4,1,4) \\
\mathbb{P}^{2}, \text { Diff }=\left(0, \frac{2}{3}, 0, \frac{n-1}{n}\right)+\frac{1}{2} \Gamma+\frac{7}{8} \Gamma_{3}\end{array}$ & $\begin{array}{c}8,12,16 \\
18,24\end{array}$ \\
\hline 2 & $\begin{array}{c}x^{5}+a z x^{3} y^{3}+b z x y^{12}+ \\
c x^{3} y^{9}+d x y^{18},|b|+|d| \neq 0\end{array}$ & $\begin{array}{l}t+z^{3} x+g\left(z, x, y^{1 / 3}\right) \subset \mathbb{P}(15,4,3,2) \\
\mathbb{P}(2,3,1), \text { Diff }=\left(0,0,0, \frac{2}{3}\right)+\frac{1}{2} \Gamma+\frac{3}{4} \Gamma_{3}\end{array}$ & 4 \\
\hline 3 & $\begin{array}{c}x^{5}+a z x y^{2 n}+b x y^{3 n} \\
n=3,5,7\end{array}$ & $\begin{array}{c}t+z^{3} x+g\left(z, x, y^{1 / n}\right) \subset \mathbb{P}(15,4,3,4) \\
\mathbb{P}(1,3,1), \text { Diff }=\left(0,0,0, \frac{n-1}{n}\right)+\frac{1}{2} \Gamma+\frac{7}{8} \Gamma_{3}\end{array}$ & 8 \\
\hline 4 & $\begin{array}{c}x^{5}+a x^{3} y^{n}+b x y^{2 n} \\
n=5 b \neq 0 ; 7,11\end{array}$ & $\begin{array}{l}t+z x+x^{5}+a x^{3} y+b x y^{2} \subset \mathbb{P}(5,4,1,2) \\
\mathbb{P}(2,1,1), \text { Diff }=\left(0, \frac{2}{3}, 0, \frac{n-1}{n}\right)+\frac{1}{2} \Gamma+\frac{3}{4} \Gamma_{3}\end{array}$ & 6,12 \\
\hline 5 & $\begin{array}{c}x^{5}+z x y^{n} \\
n=5,7 ; 9 ; 11,13,15\end{array}$ & $\begin{array}{c}t+z^{3} x+x^{5}+z x y \subset \mathbb{P}(15,4,3,8) \\
\mathbb{P}(1,3,2), \text { Diff }=\left(0,0,0, \frac{n-1}{n}\right)+\frac{1}{2} \Gamma+\frac{7}{8} \Gamma_{3}\end{array}$ & $8,10,16$ \\
\hline 6 & $\begin{array}{c}x^{6}+a z x y^{2 n}+b x y^{3 n} \\
n=3,4\end{array}$ & $\begin{array}{c}t^{2}+z^{3} x+g\left(z, x, y^{1 / n}\right) \subset \mathbb{P}(9,5,3,5) \\
\text { Diff }=\left(0,0,0, \frac{n-1}{n}\right)+\frac{4}{5} \Gamma_{3}\end{array}$ & 5 \\
\hline 7 & $\begin{aligned} & x^{6}+x y^{n} \\
& n= 7 ; 8 ; 11 ; 13,14 \\
&\end{aligned}$ & $\begin{array}{c}t^{2}+z x+x^{6}+x y \subset \mathbb{P}(3,5,1,5) \\
\text { Diff }=\left(0, \frac{2}{3}, 0, \frac{n-1}{n}\right)+\frac{4}{5} \Gamma_{3}\end{array}$ & $\begin{array}{c}7,9,12 \\
15 \\
\end{array}$ \\
\hline 8 & $\begin{array}{c}x^{6}+z x y^{n} \\
n=7 ; 9\end{array}$ & $\begin{array}{c}t^{2}+z^{3} x+x^{6}+z x y \subset \mathbb{P}(9,5,3,10) \\
\text { Diff }=\left(0,0,0, \frac{n-1}{n}\right)+\frac{4}{5} \Gamma_{3}\end{array}$ & 7,10 \\
\hline 9 & $\begin{array}{c}x^{5} y+a z x y^{2 n}+b x y^{3 n} \\
n=4,6\end{array}$ & $\begin{array}{c}t+z^{3} x+g(z, x, y) \subset \mathbb{P}(15 n-1,4 n, 3 n-1,4) \\
\mathbb{P}(n, 3 n-1,1), \text { Diff }=\frac{1}{2} \Gamma+\frac{7}{8} \Gamma_{3}\end{array}$ & 8 \\
\hline 10 & $\begin{array}{l}x^{5} y+a x^{3} y^{n}+b x y^{2 n-1} \\
(n=4 ; 6) \& b \neq 0 ; 10\end{array}$ & $\begin{array}{l}t+z x+g(z, x, y) \subset \mathbb{P}(5 n-3,4 n-2, n-1,2) \\
\mathbb{P}(2 n-1, n-1,1), \text { Diff }=\left(0, \frac{2}{3}, 0,0\right)+\frac{1}{2} \Gamma+\frac{3}{4} \Gamma_{3}\end{array}$ & $4,6,12$ \\
\hline 11 & $\begin{aligned} & x^{5} y+x y^{n} \\
n= & 8 ; 10 ; 14 ; 16 ; 20\end{aligned}$ & $\begin{array}{c}t+z x+g(z, x, y) \subset \mathbb{P}(5 n-1,4 n, n-1,4) \\
\mathbb{P}(n, n-1,1), \operatorname{Diff}=\left(0, \frac{2}{3}, 0,0\right)+\frac{1}{2} \Gamma+\frac{7}{8} \Gamma_{3}\end{array}$ & $\begin{array}{c}9,12,16 \\
18,24\end{array}$ \\
\hline
\end{tabular}




\begin{tabular}{|c|c|c|c|}
\hline 12 & $\begin{array}{c}x^{5} y+a z x^{3} y^{3}+b z x y^{10}+ \\
c x^{3} y^{8}+d x y^{15}\end{array}$ & $\begin{array}{c}t+z^{3} x+g(z, x, y) \subset \mathbb{P}(37,10,7,2) \\
\mathbb{P}(5,7,1), \text { Diff }=\frac{1}{2} \Gamma+\frac{3}{4} \Gamma_{3}\end{array}$ & 4 \\
\hline 13 & $\begin{array}{c}x^{5} y+z x y^{n} \\
n=5,7 ; 9 ; 11,13\end{array}$ & $\begin{array}{c}t+z^{3} x+g(z, x, y) \subset \mathbb{P}(15 n-2,4 n, 3 n-2,8) \\
\mathbb{P}(n, 3 n-2,2), \text { Diff }=\frac{1}{2} \Gamma+\frac{7}{8} \Gamma_{3}\end{array}$ & $8,10,16$ \\
\hline 14 & $\begin{array}{c}\frac{z x^{5}}{n}+\underline{x^{7}}+x y^{n} \\
n=7\end{array}$ & $\begin{array}{c}t+z^{3} x+g\left(z, x, y^{1 / n}\right) \subset \mathbb{P}(7,2,1,6) \\
\mathbb{P}(1,1,3), \text { Diff }=\left(0,0,0, \frac{n-1}{n}\right)+\frac{1}{2} \Gamma+\frac{3}{4} \Gamma_{3}\end{array}$ & 8,12 \\
\hline 15 & $\begin{array}{c}\underline{a} z x^{2 i+1} y^{3 k} f_{2-i-k}\left(x^{2}, y^{3}\right)+ \\
+\underline{b} x^{2 j+1} y^{3 l} f_{3-j-l}\left(x^{2}, y^{3}\right) \\
(k=0 \| l \leq 1) \&(i=0 \| j=0)\end{array}$ & $\begin{array}{c}t+z^{3} x+g\left(z, x, y^{1 / 3}\right) \subset \mathbb{P}(7,2,1,2) \\
\mathbb{P}^{2}, \text { Diff }=\left(0,0,0, \frac{2}{3}\right)+\frac{1}{2} \Gamma+\frac{3}{4} \Gamma_{3}\end{array}$ & 6 \\
\hline 16 & $\begin{array}{c}\underline{z x^{5}}+\underline{x^{7}}+a z x y^{n}+b x^{3} y^{n} \\
n=5 a \neq 0 ; 7\end{array}$ & $\begin{array}{c}t+z^{3} x+g\left(z, x, y^{1 / n}\right) \subset \mathbb{P}(7,2,1,4) \\
\mathbb{P}(1,1,2), \text { Diff }=\left(0,0,0, \frac{n-1}{n}\right)+\frac{1}{2} \Gamma+\frac{3}{4} \Gamma_{3}\end{array}$ & 6,8 \\
\hline 17 & $\begin{array}{c}x^{6} y+x y^{n} \\
n=7,8 ; 10 ; 13\end{array}$ & $\begin{array}{c}t+z x+g(z, x, y) \subset \mathbb{P}(6 n-1,5 n, n-1,5) \\
\mathbb{P}(n, n-1,1), \text { Diff }=\left(0, \frac{2}{3}, 0,0\right)+\frac{1}{2} \Gamma+\frac{9}{10} \Gamma_{3}\end{array}$ & $10,12,30$ \\
\hline 18 & $\begin{array}{c}x^{6} y+a z x y^{2 n}+b x y^{3 n} \\
n=3,4\end{array}$ & $\begin{array}{c}t+z^{3} x+g(z, x, y) \subset \mathbb{P}(18 n-1,5 n, 3 n-1,5) \\
\mathbb{P}(n, 3 n-1,1), \text { Diff }=\frac{1}{2} \Gamma+\frac{9}{10} \Gamma_{3}\end{array}$ & 10 \\
\hline 19 & $\begin{array}{c}x^{6} y+z x y^{n} \\
n=5,7\end{array}$ & $\begin{array}{c}t^{2}+z^{3} x+g(z, x, y) \subset \mathbb{P}(9 n-1,5 n, 3 n-2,10) \\
\text { Diff }=\frac{4}{5} \Gamma_{3}\end{array}$ & 5 \\
\hline 20 & $\begin{array}{c}x^{5} y^{2}+x y^{n} \\
n=7 ; 11 ; 13 ; 17\end{array}$ & $\begin{array}{c}t+z x+g(z, x, y) \subset \mathbb{P}(5 n-2,4 n, n-2,4) \\
\mathbb{P}(n, n-2,1), \text { Diff }=\left(0, \frac{2}{3}, 0,0\right)+\frac{1}{2} \Gamma+\frac{7}{8} \Gamma_{3}\end{array}$ & $\begin{array}{l}9,16,18 \\
24\end{array}$ \\
\hline 21 & $\begin{array}{c}x^{5} y^{2}+a x^{3} y^{n}+b x y^{2 n-2} \\
n=5 b \neq 0 ; 9\end{array}$ & $\begin{array}{c}t+z x+g(z, x, y) \subset \mathbb{P}(5 n-6,4 n-4, n-2,2) \\
\mathbb{P}(2 n-2, n-2,1), \text { Diff }=\left(0, \frac{2}{3}, 0,0\right)+\frac{1}{2} \Gamma+\frac{3}{4} \Gamma_{3}\end{array}$ & 6,12 \\
\hline 22 & $\begin{array}{c}x^{5} y^{2}+a z x y^{2 n}+b x y^{3 n} \\
n=3,5\end{array}$ & $\begin{array}{c}t+z^{3} x+g(z, x, y) \subset \mathbb{P}(15 n-2,4 n, 3 n-2,4) \\
\mathbb{P}(n, 3 n-2,1), \text { Diff }=\frac{1}{2} \Gamma+\frac{7}{8} \Gamma_{3}\end{array}$ & 8 \\
\hline 23 & $\begin{array}{c}x^{5} y^{2}+a z x^{3} y^{3}+b z x y^{8}+ \\
c x^{3} y^{7}+d x y^{12}\end{array}$ & $\begin{array}{c}t+z^{3} x+g(z, x, y) \subset \mathbb{P}(29,8,5,2) \\
\mathbb{P}(4,5,1), \text { Diff }=\frac{1}{2} \Gamma+\frac{3}{4} \Gamma_{3}\end{array}$ & 4 \\
\hline 24 & $\begin{array}{l}x^{5} y^{2}+z x y^{n} \\
n=5 ; 7 ; 9,11\end{array}$ & $\begin{array}{c}t+z^{3} x+g(z, x, y) \subset \mathbb{P}(15 n-4,4 n, 3 n-4,8) \\
\mathbb{P}(n, 3 n-4,2), \text { Diff }=\frac{1}{2} \Gamma+\frac{7}{8} \Gamma_{3}\end{array}$ & $8,10,16$ \\
\hline 25 & $\begin{array}{l}x^{8}+x y^{n} \\
n=8 ; 10\end{array}$ & $\begin{array}{c}t^{2}+z x+x^{8}+x y \subset \mathbb{P}(4,7,1,7) \\
\text { Diff }=\left(0, \frac{2}{3}, 0, \frac{n-1}{n}\right)+\frac{6}{7} \Gamma_{3}\end{array}$ & 9,21 \\
\hline 26 & $x^{8}+a z x y^{6}+b x y^{9}$ & $\begin{array}{c}t^{2}+z^{3} x+x^{8}+a z x y^{2}+b x y^{3} \subset \mathbb{P}(12,7,3,7) \\
\text { Diff }=\left(0,0,0, \frac{2}{3}\right)+\frac{6}{7} \Gamma_{3}\end{array}$ & 7 \\
\hline 27 & $x^{8}+z x y^{5}$ & $\begin{array}{c}t^{2}+z^{3} x+x^{8}+z x y \subset \mathbb{P}(12,7,3,14) \\
\text { Diff }=\left(0,0,0, \frac{4}{5}\right)+\frac{6}{7} \Gamma_{3}\end{array}$ & 7 \\
\hline 28 & $x^{7} y+a z x^{3} y^{3}+x y^{8}$ & $\begin{array}{c}t+z^{3} x+g(z, x, y) \subset \mathbb{P}(55,16,7,6) \\
\mathbb{P}(8,7,3), \text { Diff }=\frac{1}{2} \Gamma+\frac{3}{4} \Gamma_{3}\end{array}$ & 4 \\
\hline 29 & $\frac{x^{7} y}{b x^{4} y^{5}+c x y^{9}}+\frac{z x^{4} y^{2}+a z x y^{6}+}{}$ & $\begin{array}{c}t+z^{3} x+g(z, x, y) \subset \mathbb{P}(31,9,4,3) \\
\mathbb{P}(3,4,1), \text { Diff }=\frac{1}{2} \Gamma+\frac{5}{6} \Gamma_{3}\end{array}$ & 6 \\
\hline 30 & $x^{7} y+x^{5} y^{4}+a x^{3} y^{7}+b x y^{10}$ & $\begin{array}{c}t+z x+g(z, x, y) \subset \mathbb{P}(23,20,3,2) \\
\mathbb{P}(10,3,1), \text { Diff }=\left(0, \frac{2}{3}, 0,0\right)+\frac{1}{2} \Gamma+\frac{3}{4} \Gamma_{3}\end{array}$ & 12 \\
\hline 31 & $\begin{array}{c}x^{7} y+z x y^{n} \\
n=5 ; 7\end{array}$ & $\begin{array}{c}t+z^{3} x+g(z, x, y) \subset \mathbb{P}(21 n-2,6 n, 3 n-2,12) \\
\mathbb{P}(n, 3 n-2,2), \text { Diff }=\frac{1}{2} \Gamma+\frac{11}{12} \Gamma_{3}\end{array}$ & 12,24 \\
\hline 32 & $\begin{array}{l}x^{6} y^{2}+x y^{n} \\
n=8 ; 10 ; 11\end{array}$ & $\begin{array}{c}t^{2}+z x+g(z, x, y) \subset \mathbb{P}(3 n-1,5 n, n-2,5) \\
\text { Diff }=\left(0, \frac{2}{3}, 0,0\right)+\frac{4}{5} \Gamma_{3}\end{array}$ & $9,12,15$ \\
\hline 33 & $x^{6} y^{2}+a z x y^{6}+b x y^{9}$ & $t^{2}+z^{3} x+g(z, x, y) \subset \mathbb{P}(26,15,7,5)$ & 5 \\
\hline
\end{tabular}




\begin{tabular}{|c|c|c|c|}
\hline & & Diff $=\frac{4}{5} \Gamma_{3}$ & \\
\hline 34 & $\begin{array}{l}x^{6} y^{2}+z x y^{n} \\
n=5 ; 7\end{array}$ & $\begin{array}{c}t^{2}+z^{3} x+g(z, x, y) \subset \mathbb{P}(9 n-2,5 n, 3 n-4,10) \\
\text { Diff }=\frac{4}{5} \Gamma_{3}\end{array}$ & 7,10 \\
\hline 35 & $\begin{array}{c}x^{5} y^{3}+x y^{n} \\
n=8 ; 10 ; 14\end{array}$ & $\begin{array}{c}t+z x+g(z, x, y) \subset \mathbb{P}(5 n-3,4 n, n-3,4) \\
\mathbb{P}(n, n-3,1), \text { Diff }=\left(0, \frac{2}{3}, 0,0\right)+\frac{1}{2} \Gamma+\frac{7}{8} \Gamma_{3}\end{array}$ & $12,16,24$ \\
\hline 36 & $x^{5} y^{3}+a z x y^{8}+b x y^{12}$ & $\begin{array}{c}t+z^{3} x+g(z, x, y) \subset \mathbb{P}(57,16,9,4) \\
\mathbb{P}(4,9,1), \text { Diff }=\frac{1}{2} \Gamma+\frac{7}{8} \Gamma_{3}\end{array}$ & 8 \\
\hline 37 & $x^{5} y^{3}+a x^{3} y^{8}+b x y^{13}$ & $\begin{array}{c}t+z x+g(z, x, y) \subset \mathbb{P}(31,26,5,2) \\
\mathbb{P}(13,5,1), \text { Diff }=\left(0, \frac{2}{3}, 0,0\right)+\frac{1}{2} \Gamma+\frac{3}{4} \Gamma_{3}\end{array}$ & 12 \\
\hline 38 & $\begin{array}{l}x^{5} y^{3}+z x y^{n} \\
n=5 ; 7\end{array}$ & $\begin{array}{c}t+z^{3} x+g(z, x, y) \subset \mathbb{P}(15 n-6,4 n, 3 n-6,8) \\
\mathbb{P}(n, 3 n-6,2), \text { Diff }=\frac{1}{2} \Gamma+\frac{7}{8} \Gamma_{3}\end{array}$ & 8,10 \\
\hline 39 & $x^{5} y^{3}+z x y^{9}$ & $\begin{array}{c}t+z^{3} x+x^{5} y+z x y^{3} \subset \mathbb{P}(43,12,7,8) \\
\mathbb{P}(3,7,2), \text { Diff }=\left(0,0,0, \frac{2}{3}\right)+\frac{1}{2} \Gamma+\frac{7}{8} \Gamma_{3}\end{array}$ & 16 \\
\hline 40 & $x^{9}+a z x y^{6}+b x y^{9}$ & $\begin{array}{c}t+z^{3} x+x^{9}+a z x y^{2}+b x y^{3} \subset \mathbb{P}(27,8,3,8) \\
\mathbb{P}(1,3,1), \text { Diff }=\left(0,0,0, \frac{2}{3}\right)+\frac{1}{2} \Gamma+\frac{15}{16} \Gamma_{3}\end{array}$ & 16 \\
\hline 41 & $x^{9}+x^{3} y^{7}$ & $\begin{array}{c}t+z x+x^{9}+x^{3} y \subset \mathbb{P}(9,8,1,6) \\
\mathbb{P}(4,1,3), \text { Diff }=\left(0, \frac{2}{3}, 0, \frac{6}{7}\right)+\frac{1}{2} \Gamma+\frac{3}{4} \Gamma_{3}\end{array}$ & 36 \\
\hline 42 & $x^{8} y+a z x y^{6}+b x y^{9}$ & $\begin{array}{c}t+z^{3} x+g(z, x, y) \subset \mathbb{P}(71,21,8,7) \\
\mathbb{P}(3,8,1), \text { Diff }=\frac{1}{2} \Gamma+\frac{13}{14} \Gamma_{3}\end{array}$ & 14 \\
\hline 43 & $x^{7} y^{2}+a z x y^{6}+b x y^{9}$ & $\begin{array}{c}t+z^{3} x+g(z, x, y) \subset \mathbb{P}(61,18,7,6) \\
\mathbb{P}(3,7,1), \text { Diff }=\frac{1}{2} \Gamma+\frac{11}{12} \Gamma_{3}\end{array}$ & 12 \\
\hline 44 & $x^{7} y^{2}+x^{3} y^{7}$ & $\begin{array}{c}t+z x+g(z, x, y) \subset \mathbb{P}(43,38,5,4) \\
\mathbb{P}(19,5,2), \text { Diff }=\left(0, \frac{2}{3}, 0,0\right)+\frac{1}{2} \Gamma+\frac{3}{4} \Gamma_{3}\end{array}$ & 24 \\
\hline 45 & $z x^{6}+x^{6} y^{3}+a z x y^{6}+b x y^{9}$ & $\begin{array}{l}t+z^{3} x+g\left(z, x, y^{1 / 3}\right) \subset \mathbb{P}(17,5,2,5) \\
\mathbb{P}(1,2,1), \text { Diff }=\left(0,0,0, \frac{2}{3}\right)+\frac{1}{2} \Gamma+\frac{9}{10} \Gamma_{3}\end{array}$ & 10 \\
\hline 46 & $x^{6} y^{3}+x y^{10}$ & $\begin{array}{c}t+z x+g(z, x, y) \subset \mathbb{P}(57,50,7,5) \\
\mathbb{P}(10,7,1), \text { Diff }=\left(0, \frac{2}{3}, 0,0\right)+\frac{1}{2} \Gamma+\frac{9}{10} \Gamma_{3}\end{array}$ & 30 \\
\hline 47 & $z x^{5} y+x^{5} y^{4}+a z x y^{6}+b x y^{9}$ & $\begin{array}{c}t+z^{3} x+g(z, x, y) \subset \mathbb{P}(41,12,5,4) \\
\mathbb{P}(3,5,1), \text { Diff }=\frac{1}{2} \Gamma+\frac{7}{8} \Gamma_{3}\end{array}$ & 8 \\
\hline 48 & $x^{5} y^{4}+x y^{11}$ & $\begin{array}{c}t+z x+g(z, x, y) \subset \mathbb{P}(51,44,7,4) \\
\mathbb{P}(11,7,1), \text { Diff }=\left(0, \frac{2}{3}, 0,0\right)+\frac{1}{2} \Gamma+\frac{7}{8} \Gamma_{3}\end{array}$ & 24 \\
\hline 49 & $x^{5} y^{4}+z x y^{7}$ & $\begin{array}{c}t+z^{3} x+g(z, x, y) \subset \mathbb{P}(97,28,13,8) \\
\mathbb{P}(7,13,2), \text { Diff }=\frac{1}{2} \Gamma+\frac{7}{8} \Gamma_{3}\end{array}$ & 16 \\
\hline 50 & $\begin{array}{c}z x^{4}+x y^{n} \\
n=7 ; 11 ; 13 ; 17\end{array}$ & $\begin{array}{c}t+z^{3} x+z x^{4}+x y \subset \mathbb{P}(11,3,2,9) \\
\mathbb{P}(1,2,3), \text { Diff }=\left(0,0,0, \frac{n-1}{n}\right)+\frac{1}{2} \Gamma+\frac{5}{6} \Gamma_{3}\end{array}$ & $\begin{array}{c}7,12,14 \\
18\end{array}$ \\
\hline 51 & $z x^{4}+a z x y^{8}+b x^{4} y^{4}+c x y^{12}$ & $\begin{array}{c}t^{2}+z^{3} x+g\left(z, x, y^{1 / 2}\right) \subset \mathbb{P}(11,6,4,3) \\
\text { Diff }=\left(0,0,0, \frac{1}{2}\right)+\frac{2}{3} \Gamma_{3}\end{array}$ & 3 \\
\hline 52 & $\begin{array}{c}z x^{4}+x y^{2 n} \\
n=4,5 ; 7,8\end{array}$ & $\begin{array}{c}t^{2}+z^{3} x+z x^{4}+x y^{2} \subset \mathbb{P}(11,6,4,9) \\
\text { Diff }=\left(0,0,0, \frac{n-1}{n}\right)+\frac{2}{3} \Gamma_{3}\end{array}$ & 5,9 \\
\hline$\overline{53}$ & $\begin{array}{c}z x^{4}+a z x y^{10}+b x^{4} y^{5}+ \\
c x y^{15}\end{array}$ & $\begin{array}{c}t+z^{3} x+g\left(z, x, y^{1 / 5}\right) \subset \mathbb{P}(11,3,2,3) \\
\mathbb{P}(1,2,1), \text { Diff }=\left(0,0,0, \frac{4}{5}\right)+\frac{1}{2} \Gamma+\frac{5}{6} \Gamma_{3}\end{array}$ & 6 \\
\hline 54 & $z x^{i} f_{4-i}\left(x, y^{3}\right)$ & $\begin{array}{l}t+z^{3} x+z x^{i} f_{4-i}(x, y) \subset \mathbb{P}(11,3,2,2) \\
\mathbb{P}(3,1,1), \text { Diff }=\left(0,0,0, \frac{n-1}{n}\right)+\frac{1}{2} \Gamma+\frac{3}{4} \Gamma_{2}\end{array}$ & 4 \\
\hline 55 & $z x^{4}+z y^{n}$ & $t+z^{3} x+z x^{4}+z y \subset \mathbb{P}(11,3,2,8)$ & $5,8,10$ \\
\hline
\end{tabular}




\begin{tabular}{|c|c|c|c|}
\hline & $n=5 ; 7 ; 9 ; 11 ; 13,15$ & $\mathbb{P}(3,1,4)$, Diff $=\left(0,0,0, \frac{n-1}{n}\right)+\frac{1}{2} \Gamma+\frac{3}{4} \Gamma_{2}$ & 12,16 \\
\hline 56 & $\begin{array}{l}z x^{4}+a z x^{2} y^{n}+b z y^{2 n} \\
\quad n=3 b \neq 0 ; 5,7\end{array}$ & $\begin{array}{l}t+z^{3} x+g\left(z, x, y^{1 / n}\right) \subset \mathbb{P}(11,3,2,4) \\
\mathbb{P}(3,1,2), \text { Diff }=\left(0,0,0, \frac{n-1}{n}\right)+\frac{1}{2} \Gamma+\frac{3}{4} \Gamma_{2}\end{array}$ & 4,8 \\
\hline 57 & $\begin{array}{l}z x^{4}+z x y^{n} \\
n=5 ; 7 ; 11\end{array}$ & $\begin{array}{c}t+z^{3} x+z x^{4}+z x y \subset \mathbb{P}(11,3,2,6) \\
\mathbb{P}^{2}, \text { Diff }=\left(0,0,0, \frac{n-1}{n}\right)+\frac{1}{2} \Gamma+\frac{3}{4} \Gamma_{2}+\frac{5}{6} \Gamma_{3}\end{array}$ & $6,8,12$ \\
\hline 58 & $\begin{array}{l}z x^{4} y+x y^{2 n+1} \\
\quad n=3 ; 5,6\end{array}$ & $\begin{aligned} t^{2}+z^{3} x+g(z, x, y) & \subset \mathbb{P}(11 n+4,6 n+3,4 n-1,9) \\
& \text { Diff }=\frac{2}{3} \Gamma_{3}\end{aligned}$ & 5,9 \\
\hline 59 & $\begin{array}{l}z x^{4} y+x y^{n} \\
n=8 ; 10 ; 14\end{array}$ & $\begin{array}{c}t+z^{3} x+g(z, x, y) \subset \mathbb{P}(11 n-3,3 n, 2 n-3,9) \\
\mathbb{P}(n, 2 n-3,3), \text { Diff }=\frac{1}{2} \Gamma+\frac{5}{6} \Gamma_{3}\end{array}$ & $10,12,18$ \\
\hline 60 & $\begin{array}{c}z x^{4} y+a z x y^{6}+b x^{4} y^{4}+ \\
c x y^{9}\end{array}$ & $\begin{array}{c}t^{2}+z^{3} x+g(z, x, y) \subset \mathbb{P}(16,9,5,3) \\
\text { Diff }=\frac{2}{3} \Gamma_{3}\end{array}$ & 3 \\
\hline 61 & $\begin{array}{c}z x^{4} y+a z x y^{8}+b x^{4} y^{5}+ \\
c x y^{12}\end{array}$ & $\begin{array}{c}t+z^{3} x+g(z, x, y) \subset \mathbb{P}(43,12,7,3) \\
\mathbb{P}(4,7,1), \text { Diff }=\frac{1}{2} \Gamma+\frac{5}{6} \Gamma_{3}\end{array}$ & 6 \\
\hline 62 & $\begin{array}{c}z x^{4} y+z y^{n} \\
n=6 ; 8 ; 10 ; 12\end{array}$ & $\begin{array}{c}t+z^{3} x+g(z, x, y) \subset \mathbb{P}(11 n+1,3 n+1,2 n-2,8) \\
\mathbb{P}(3 n+1, n-1,4), \text { Diff }=\frac{1}{2} \Gamma+\frac{3}{4} \Gamma_{2}\end{array}$ & $\begin{array}{c}8,10,12 \\
16\end{array}$ \\
\hline 63 & $\begin{array}{c}z x^{4} y+a z x^{2} y^{n}+b z y^{2 n-1} \\
n=4 \& b \neq 0 ; 6\end{array}$ & $\begin{array}{c}t+z^{3} x+g(z, x, y) \subset \mathbb{P}(11 n-5,3 n-1,2 n-2,4) \\
\mathbb{P}(3 n-1, n-1,2), \text { Diff }=\frac{1}{2} \Gamma+\frac{3}{4} \Gamma_{2}\end{array}$ & 4,8 \\
\hline 64 & $z y x^{i} f_{4-i}\left(x, y^{2}\right)$ & $\begin{array}{c}t+z^{3} x+z x^{i} f_{4-i}(x, y) \subset \mathbb{P}(25,7,4,2) \\
\mathbb{P}(7,2,1), \text { Diff }=\frac{1}{2} \Gamma+\frac{3}{4} \Gamma_{2}\end{array}$ & 4 \\
\hline 65 & $\begin{array}{l}z x^{4} y+z x y^{n} \\
\quad n=5 ; 9\end{array}$ & $\begin{array}{c}t+z^{3} x+g(z, x, y) \subset \mathbb{P}(11 n-2,3 n, 2 n-2,6) \\
\mathbb{P}(n, n-1,1), \text { Diff }=\frac{1}{2} \Gamma+\frac{3}{4} \Gamma_{2}+\frac{5}{6} \Gamma_{3}\end{array}$ & 6,12 \\
\hline 66 & $z x^{6}+x y^{8}$ & $\begin{array}{c}t^{2}+z^{3} x+z x^{6}+x y^{2} \subset \mathbb{P}(17,10,4,15) \\
\text { Diff }=\left(0,0,0, \frac{3}{4}\right)+\frac{4}{5} \Gamma_{3}\end{array}$ & 5 \\
\hline 67 & $\begin{array}{l}z x^{i}(x+y)^{j} y^{k} f_{6-i}(x, y) \\
i \leq 1 \& j \leq 2 \& k \leq 2\end{array}$ & $\begin{array}{c}t+z^{3} x+g(z, x, y) \subset \mathbb{P}(17,5,2,2) \\
\mathbb{P}(5,1,1), \text { Diff }=\frac{1}{2} \Gamma+\frac{3}{4} \Gamma_{2}\end{array}$ & 4 \\
\hline 68 & $z x^{6}+z y^{7}$ & $\begin{array}{l}t+z^{3} x+z x^{6}+z y \subset \mathbb{P}(17,5,2,12) \\
\mathbb{P}(5,1,6), \text { Diff }=\left(0,0,0, \frac{6}{7}\right)+\frac{1}{2} \Gamma+\frac{3}{4} \Gamma_{2}\end{array}$ & 8 \\
\hline 69 & $z x^{6}+z x^{2} y^{5}$ & $\begin{array}{l}t+z^{3} x+z x^{6}+z x^{2} y \subset \mathbb{P}(17,5,2,8) \\
\mathbb{P}(5,1,4), \text { Diff }=\left(0,0,0, \frac{4}{5}\right)+\frac{1}{2} \Gamma+\frac{3}{4} \Gamma_{2}\end{array}$ & 16 \\
\hline 70 & $\begin{array}{l}z x^{5} y+x y^{n} \\
n=8 ; 10\end{array}$ & $\begin{array}{c}t+z^{3} x+g(z, x, y) \subset \mathbb{P}(14 n-3,4 n, 2 n-3,12) \\
\mathbb{P}(n, 2 n-3,3), \text { Diff }=\frac{1}{2} \Gamma+\frac{7}{8} \Gamma_{3}\end{array}$ & 10,24 \\
\hline 71 & $z x^{5} y+x^{3} y^{7}$ & $\begin{array}{c}t+z^{3} x+z x^{5} y+x^{3} y^{7} \subset \mathbb{P}(89,26,11,8) \\
\mathbb{P}(13,11,4), \text { Diff }=\frac{1}{2} \Gamma+\frac{3}{4} \Gamma_{3}\end{array}$ & 16 \\
\hline 72 & $\begin{array}{c}z x^{5} y+z y^{n} \\
n=7 ; 8\end{array}$ & $\begin{array}{c}t+z^{3} x+g(z, x, y) \subset \mathbb{P}(14 n+1,4 n+1,2 n-2,10) \\
\mathbb{P}(4 n+1, n-1,5), \text { Diff }=\frac{1}{2} \Gamma+\frac{3}{4} \Gamma_{2}\end{array}$ & 8,20 \\
\hline 73 & $z x^{5} y+z x^{2} y^{5}$ & $\begin{array}{c}t+z^{3} x+g(z, x, y) \subset \mathbb{P}(65,19,8,6) \\
\mathbb{P}(19,4,3), \text { Diff }=\frac{1}{2} \Gamma+\frac{3}{4} \Gamma_{2}\end{array}$ & 12 \\
\hline 74 & $z x^{4} y^{2}+x y^{8}$ & $\begin{array}{c}t^{2}+z^{3} x+g(z, x, y) \subset \mathbb{P}(41,24,10,9) \\
\text { Diff }=\frac{2}{3} \Gamma_{3}\end{array}$ & 5 \\
\hline 75 & $z x^{4} y^{2}+x y^{10}$ & $\begin{array}{c}t^{2}+z^{3} x+z x^{4} y+x y^{5} \subset \mathbb{P}(26,15,7,9) \\
\text { Diff }=\left(0,0,0, \frac{1}{2}\right)+\frac{2}{3} \Gamma_{3}\end{array}$ & 9 \\
\hline 76 & $z x^{4} y^{2}+x y^{11}$ & $\begin{array}{c}t+z^{3} x+z x^{4} y^{2}+x y^{11} \subset \mathbb{P}(115,33,16,9) \\
\mathbb{P}(11,16,3), \text { Diff }=\frac{1}{2} \Gamma+\frac{5}{6} \Gamma_{3}\end{array}$ & 18 \\
\hline 77 & $z x^{4} y^{2}+z y^{n}$ & $t+z^{3} x+g(z, x, y) \subset \mathbb{P}(11 n+2,3 n+2,2 n-4,8)$ & 12,16 \\
\hline
\end{tabular}




\begin{tabular}{|c|c|c|c|}
\hline & $n=7 ; 9$ & $\mathbb{P}(3 n+2, n-2,4)$, Diff $=\frac{1}{2} \Gamma+\frac{3}{4} \Gamma_{2}$ & \\
\hline 78 & $z x^{4} y^{2}+a z x^{2} y^{5}+b z y^{8}$ & $t+z^{3} x+g(z, x, y) \subset \mathbb{P}(45,13,6,4)$ & 8 \\
& & $\mathbb{P}(13,3,2)$, Diff $=\frac{1}{2} \Gamma+\frac{3}{4} \Gamma_{2}$ & \\
\hline 79 & $z x^{4} y^{2}+z x y^{7}$ & $t+z^{3} x+z x^{4} y^{2}+z x y^{7} \subset \mathbb{P}(73,21,10,6)$ & 12 \\
& & $\mathbb{P}(7,5,1)$, Diff $=\frac{1}{2} \Gamma+\frac{3}{4} \Gamma_{2}+\frac{5}{6} \Gamma_{3}$ & \\
\hline
\end{tabular}

4. Singularity $-t^{2}+z^{3} y+g(z, x, y)$

\begin{tabular}{|c|c|c|c|}
\hline 1 & $\begin{array}{c}x^{7} y+y^{n} \\
n=9 ; 11 ; 17 ; 21\end{array}$ & $\begin{array}{c}t+z y+x y+y^{n} \subset \mathbb{P}(n, n-1, n-1,1) \\
\mathbb{P}^{2}, \text { Diff }=\left(0, \frac{2}{3}, \frac{6}{7}, 0\right)+\frac{1}{2} \Gamma+\frac{2 n-3}{2 n-2} \Gamma_{4}\end{array}$ & $\begin{array}{c}16,21,36 \\
42\end{array}$ \\
\hline 2 & $\begin{array}{c}x^{7} y+a z y^{4 n+3}+b y^{6 n+4} \\
n=1,2\end{array}$ & $\begin{array}{c}t^{2}+z^{3} y+g\left(z, x^{1 / 7}, y\right) \subset \mathbb{P}(3 n+2,2 n+1,6 n+3,1) \\
\text { Diff }=\left(0,0, \frac{6}{7}, 0\right)+\frac{2 n}{2 n+1} \Gamma_{4}\end{array}$ & 7 \\
\hline 3 & $\begin{array}{c}x^{7} y+y^{2 n} \\
n=6,7 ; 9,10 \\
\end{array}$ & $\begin{array}{c}t^{2}+z y+x y+y^{2 n} \subset \mathbb{P}(n, 2 n-1,2 n-1,1) \\
\text { Diff }=\left(0, \frac{2}{3}, \frac{6}{7}, 0\right)+\frac{2 n-2}{2 n-1} \Gamma_{4}\end{array}$ & 15,21 \\
\hline 4 & $\begin{array}{c}x^{7} y+a z y^{2 n+1}+b y^{3 n+1} \\
n=4 ; 6\end{array}$ & $\begin{array}{l}t+z^{3} y+g\left(z, x^{1 / 7}, y\right) \subset \mathbb{P}(3 n+1, n, 3 n, 1) \\
\mathbb{P}(1,3,1), \text { Diff }=\left(0,0, \frac{6}{7}, 0\right)+\frac{1}{2} \Gamma+\frac{2 n-1}{2 n} \Gamma_{4}\end{array}$ & 8,14 \\
\hline 5 & $y x^{i} f_{7-i}\left(x, y^{2}\right)$ & $\begin{array}{c}t+z y+y x^{i} f_{7-i}\left(x, y^{2}\right) \subset \mathbb{P}(15,14,2,1) \\
\mathbb{P}(7,1,1), \text { Diff }=\left(0, \frac{2}{3}, 0,0\right)+\frac{1}{2} \Gamma+\frac{3}{4} \Gamma_{4}\end{array}$ & 6 \\
\hline 6 & $\begin{array}{c}x^{7} y+x y^{n} \\
n=8 ; 12 ; 14 ; 18\end{array}$ & $\begin{array}{c}t+z y+x^{7} y+x y^{n} \subset \mathbb{P}(7 n-1,7 n-7, n-1,6) \\
\mathbb{P}(7,1,6), \text { Diff }=\left(0, \frac{2}{3}, 0,0\right)+\frac{1}{2} \Gamma+\frac{2 n-3}{2 n-2} \Gamma_{4}\end{array}$ & $\begin{array}{l}15,22,30 \\
36\end{array}$ \\
\hline 7 & $\begin{array}{c}x^{7} y+a x^{4} y^{n}+b x y^{2 n-1} \\
n=5 ; 9\end{array}$ & $\begin{array}{c}t+z y+g(z, x, y) \subset \mathbb{P}(7 n-4,7 n-7, n-1,3) \\
\mathbb{P}(7,1,3), \text { Diff }=\left(0, \frac{2}{3}, 0,0\right)+\frac{1}{2} \Gamma+\frac{2 n-3}{2 n-2} \Gamma_{4}\end{array}$ & 12,18 \\
\hline 8 & $\begin{array}{c}x^{7} y+a x^{4} y^{2 n}+b x y^{4 n-1} \\
n=3,4\end{array}$ & $\begin{array}{c}t^{2}+z y+g(z, x, y) \subset \mathbb{P}(7 n-2,14 n-7,2 n-1,3) \\
\mathbb{P}(7,1,3), \text { Diff }=\left(0, \frac{2}{3}, 0,0\right)+\frac{2 n-2}{2 n-1} \Gamma_{4} \\
\end{array}$ & 9 \\
\hline 9 & $\begin{array}{c}x^{7} y+a x^{5} y^{6}+b x^{3} y^{11}+ \\
c x y^{16}\end{array}$ & $\begin{array}{c}t+z y+g(z, x, y) \subset \mathbb{P}(37,35,5,2) \\
\mathbb{P}(7,1,2), \text { Diff }=\left(0, \frac{2}{3}, 0,0\right)+\frac{1}{2} \Gamma+\frac{9}{10} \Gamma_{4} \\
\end{array}$ & 12 \\
\hline$\overline{10}$ & $\begin{array}{c}x^{7} y+a z x^{3} y^{n}+b x^{2} y^{3 n-2} \\
n=3 b \neq 0 ; 5\end{array}$ & $\begin{array}{c}t+z^{3} y+g(z, x, y) \subset \mathbb{P}(21 n-16,7 n-7,3 n-3,5) \\
\mathbb{P}(7,3,5), \text { Diff }=\frac{1}{2} \Gamma+\frac{2 n-3}{2 n-2} \Gamma_{4}\end{array}$ & 4,10 \\
\hline 11 & $\begin{array}{c}x^{7} y+x^{2} y^{2 n} \\
n=4 ; 6,7\end{array}$ & $\begin{array}{c}t^{2}+z y+g(z, x, y) \subset \mathbb{P}(7 n-1,14 n-7,2 n-1,5) \\
\text { Diff }=\left(0, \frac{2}{3}, 0,0\right)+\frac{2 n-2}{2 n-1} \Gamma_{4}\end{array}$ & 9,15 \\
\hline 12 & $\begin{array}{c}x^{7} y+x^{2} y^{n} \\
n=9 ; 15\end{array}$ & $\begin{array}{c}t+z y+g(z, x, y) \subset \mathbb{P}(7 n-2,7 n-7, n-1,5) \\
\mathbb{P}(7,1,5), \text { Diff }=\left(0, \frac{2}{3}, 0,0\right)+\frac{1}{2} \Gamma+\frac{2 n-3}{2 n-2} \Gamma_{4} \\
\end{array}$ & 16,30 \\
\hline 13 & $x^{7} y+a z x^{3} y^{4}+b x^{2} y^{10}$ & $\begin{array}{c}t^{2}+z^{3} y+g(z, x, y) \subset \mathbb{P}(34,21,9,5) \\
\text { Diff }=\frac{2}{3} \Gamma_{4}\end{array}$ & 5 \\
\hline 14 & $\begin{array}{l}x^{7} y+x^{3} y^{n} \\
n=6 ; 8 ; 12\end{array}$ & $\begin{array}{c}t+z y+g(z, x, y) \subset \mathbb{P}(7 n-3,7 n-7, n-1,4) \\
\mathbb{P}(7,1,4), \text { Diff }=\left(0, \frac{2}{3}, 0,0\right)+\frac{1}{2} \Gamma+\frac{2 n-3}{2 n-2} \Gamma_{4}\end{array}$ & $10,18,24$ \\
\hline 15 & $x^{7} y+a z x^{2} y^{7}+b x^{3} y^{10}$ & $\begin{array}{c}t+z^{3} y+g(z, x, y) \subset \mathbb{P}(67,21,9,4) \\
\mathbb{P}(7,3,4), \text { Diff }=\frac{1}{2} \Gamma+\frac{5}{6} \Gamma_{4}\end{array}$ & 8 \\
\hline 16 & $\begin{array}{c}x^{7} y+z y^{n} \\
n=6 ; 10,12 ; 14\end{array}$ & $\begin{array}{c}t+z^{3} y+x y+z y^{n} \subset \mathbb{P}(3 n-1, n-1,3 n-3,2) \\
\mathbb{P}(1,3,2), \text { Diff }=\left(0,0, \frac{6}{7}, 0\right)+\frac{1}{2} \Gamma+\frac{2 n-3}{2 n-2} \Gamma_{4}\end{array}$ & $10,22,28$ \\
\hline 17 & $\begin{array}{c}x^{7} y+z x y^{n} \\
n=5 ; 7,9 ; 11\end{array}$ & $\begin{array}{c}t+z^{3} y+g(z, x, y) \subset \mathbb{P}(21 n-10,7 n-7,3 n-3,11) \\
\mathbb{P}(7,3,11), \text { Diff }=\frac{1}{2} \Gamma+\frac{2 n-3}{2 n-2} \Gamma_{4}\end{array}$ & $8,16,22$ \\
\hline 18 & $\begin{array}{c}x^{7} y+z x y^{2 n} \\
n=3 ; 4,5 \\
\end{array}$ & $\begin{array}{c}t^{2}+z^{3} y+g(z, x, y) \subset \mathbb{P}(21 n-5,14 n-7,6 n-3,11) \\
\text { Diff }=\frac{2 n-2}{2 n-1} \Gamma_{4}\end{array}$ & 5,11 \\
\hline 19 & $\begin{array}{l}x^{7} y+z x^{2} y^{n} \\
n=4,6 ; 8\end{array}$ & $\begin{array}{c}t+z^{3} y+g(z, x, y) \subset \mathbb{P}(21 n-13,7 n-7,3 n-3,8) \\
\mathbb{P}(7,3,8), \text { Diff }=\frac{1}{2} \Gamma+\frac{2 n-3}{2 n-2} \Gamma_{4}\end{array}$ & 10,16 \\
\hline
\end{tabular}




\begin{tabular}{|c|c|c|c|}
\hline 20 & $x^{8} y+a z y^{7}+b y^{10}$ & $\begin{array}{c}t^{2}+z^{3} y+x y+a z y^{7}+b y^{10} \subset \mathbb{P}(5,3,9,1) \\
\text { Diff }=\left(0,0, \frac{7}{8}, 0\right)+\frac{2}{3} \Gamma_{4}\end{array}$ & 8 \\
\hline 21 & $x^{8} y+a x^{4} y^{6}+b y^{11}$ & $\begin{array}{c}t^{2}+z y+x^{4} y+a x^{2} y^{6}+b y^{11} \subset \mathbb{P}(11,20,5,2) \\
\text { Diff }=\left(0, \frac{2}{3}, \frac{1}{2}, 0\right)+\frac{4}{5} \Gamma_{4}\end{array}$ & 12 \\
\hline 22 & $x^{8} y+y^{12}$ & $\begin{array}{c}t^{2}+z y+x y+y^{12} \subset \mathbb{P}(6,11,11,1) \\
\text { Diff }=\left(0, \frac{2}{3}, \frac{7}{8}, 0\right)+\frac{10}{11} \Gamma_{4}\end{array}$ & 24 \\
\hline 23 & $\begin{array}{c}x^{8} y+x y^{n} \\
n=9 ; 11\end{array}$ & $\begin{array}{c}t+z y+g(z, x, y) \subset \mathbb{P}(8 n-1,8 n-8, n-1,7) \\
\mathbb{P}(8,1,7), \text { Diff }=\left(0, \frac{2}{3}, 0,0\right)+\frac{1}{2} \Gamma+\frac{2 n-3}{2 n-2} \Gamma_{4}\end{array}$ & 18,42 \\
\hline 24 & $x^{8} y+a z x^{3} y^{4}+b x y^{10}$ & $\begin{array}{c}t+z^{3} y+g(z, x, y) \subset \mathbb{P}(79,24,9,7) \\
\mathbb{P}(8,3,7), \text { Diff }=\frac{1}{2} \Gamma+\frac{5}{6} \Gamma_{4}\end{array}$ & 14 \\
\hline 25 & $x^{8} y+x^{2} y^{8}$ & $\begin{array}{c}t^{2}+z y+x^{8} y+x^{2} y^{8} \subset \mathbb{P}(31,56,7,6) \\
\text { Diff }=\left(0, \frac{2}{3}, 0,0\right)+\frac{6}{7} \Gamma_{4}\end{array}$ & 9 \\
\hline 26 & $x^{8} y+a x^{5} y^{5}+b x^{2} y^{9}$ & $\begin{array}{c}t+z y+g(z, x, y) \subset \mathbb{P}(35,32,4,3) \\
\mathbb{P}(8,1,3), \text { Diff }=\left(0, \frac{2}{3}, 0,0\right)+\frac{1}{2} \Gamma+\frac{7}{8} \Gamma_{4}\end{array}$ & 18 \\
\hline 27 & $x^{8} y+a z x^{2} y^{5}+b x^{3} y^{7}$ & $\begin{array}{c}t+z^{3} y+g(z, x, y) \subset \mathbb{P}(53,16,6,5) \\
\mathbb{P}(8,3,5), \text { Diff }=\frac{1}{2} \Gamma+\frac{3}{4} \Gamma_{4}\end{array}$ & 10 \\
\hline 28 & $x^{8} y+x^{3} y^{8}$ & $\begin{array}{l}t+z y+x^{8} y+x^{3} y^{8} \subset \mathbb{P}(61,56,7,5) \\
\mathbb{P}(8,1,5), \text { Diff }=\left(0, \frac{2}{3}, 0,0\right)+\frac{1}{2} \Gamma+\frac{13}{14} \Gamma_{4}\end{array}$ & 30 \\
\hline 29 & $x^{8} y+z y^{8}$ & $\begin{array}{c}t^{2}+z^{3} y+x^{2} y+z y^{8} \subset \mathbb{P}(23,14,21,4) \\
\text { Diff }=\left(0,0, \frac{3}{4}, 0\right)+\frac{6}{7} \Gamma_{4}\end{array}$ & 16 \\
\hline 30 & $\begin{array}{c}x^{8} y+z x y^{n} \\
n=6 ; 7\end{array}$ & $\begin{array}{c}t+z^{3} y+g(z, x, y) \subset \mathbb{P}(24 n-11,8 n-8,3 n-3,13) \\
\mathbb{P}(8,3,13), \text { Diff }=\frac{1}{2} \Gamma+\frac{2 n-3}{2 n-2} \Gamma_{4}\end{array}$ & 10,26 \\
\hline 31 & $\begin{aligned} & z x^{5}+z y^{n} \\
& n=6 ; 8 ; 12 ; 14\end{aligned}$ & $\begin{array}{c}t+z^{3} y+z x+z y^{n} \subset \mathbb{P}(3 n-1, n-1,2 n, 2) \\
\mathbb{P}(n-1, n, 1), \text { Diff }=\left(0,0, \frac{4}{5}, 0\right)+\frac{1}{2} \Gamma+\frac{3}{4} \Gamma_{2}\end{array}$ & $\begin{array}{c}8,10,16 \\
20\end{array}$ \\
\hline 32 & $z x^{i} f_{5-i}\left(x, y^{2}\right)$ & $\begin{array}{c}t+z^{3} y+z x^{i} f_{5-i}\left(x, y^{2}\right) \subset \mathbb{P}(29,9,4,2) \\
\mathbb{P}(9,2,1), \text { Diff }=\frac{1}{2} \Gamma+\frac{3}{4} \Gamma_{2}\end{array}$ & 4 \\
\hline 33 & $\begin{array}{c}z x^{5}+z x y^{n} \\
n=5 ; 7,9 ; 11\end{array}$ & $\begin{array}{c}t+z^{3} y+g(z, x, y) \subset \mathbb{P}(15 n-4,5 n-4,2 n, 8) \\
\mathbb{P}(5 n-4, n, 4), \text { Diff }=\frac{1}{2} \Gamma+\frac{3}{4} \Gamma_{2}\end{array}$ & $6,12,16$ \\
\hline 34 & $\begin{array}{c}z x^{5}+a z x^{3} y^{n}+b z x y^{2 n} \\
n=3 b \neq 0 ; 5\end{array}$ & $\begin{array}{c}t+z^{3} y+g(z, x, y) \subset \mathbb{P}(15 n-2,5 n-2,2 n, 4) \\
\mathbb{P}(5 n-2, n, 2), \text { Diff }=\frac{1}{2} \Gamma+\frac{3}{4} \Gamma_{2}\end{array}$ & 4,8 \\
\hline 35 & $\begin{array}{c}z x^{5}+z x^{2} y^{n} \\
n=4 ; 8\end{array}$ & $\begin{array}{c}t+z^{3} y+g(z, x, y) \subset \mathbb{P}(15 n-3,5 n-3,2 n, 6) \\
\mathbb{P}(5 n-3, n, 3), \text { Diff }=\frac{1}{2} \Gamma+\frac{3}{4} \Gamma_{2}\end{array}$ & 8,12 \\
\hline 36 & $z x^{6}+a z x^{3} y^{4}+b z y^{8}$ & $\begin{array}{c}t+z^{3} y+z x^{2}+a z x y^{4}+b z y^{8} \subset \mathbb{P}(23,7,8,2) \\
\mathbb{P}(7,4,1), \text { Diff }=\left(0,0, \frac{2}{3}, 0\right)+\frac{1}{2} \Gamma+\frac{3}{4} \Gamma_{2}\end{array}$ & 12 \\
\hline 37 & $\begin{array}{l}z x^{6}+z x y^{n} \\
n=6 ; 7\end{array}$ & $\begin{array}{c}t+z^{3} y+g(z, x, y) \subset \mathbb{P}(18 n-5,6 n-5,2 n, 10) \\
\mathbb{P}(6 n-5, n, 5), \text { Diff }=\frac{1}{2} \Gamma+\frac{3}{4} \Gamma_{2}\end{array}$ & 8,20 \\
\hline 38 & $\begin{array}{c}z x^{5} y+y^{n} \\
n=9 ; 15\end{array}$ & $\begin{array}{c}t+z^{3} y+z x y+y^{n} \subset \mathbb{P}(3 n, n-1,2 n-2,3) \\
\mathbb{P}(1,2,3), \text { Diff }=\left(0,0, \frac{4}{5}, 0\right)+\frac{1}{2} \Gamma+\frac{2 n-3}{2 n-2} \Gamma_{4}\end{array}$ & 16,30 \\
\hline 39 & $\begin{array}{c}z x^{5} y+a z y^{7}+b x^{5} y^{4}+ \\
c y^{10},|a|+|c| \neq 0\end{array}$ & $\begin{array}{c}t^{2}+z^{3} y+g\left(z, x^{1 / 5}, y\right) \subset \mathbb{P}(5,3,6,1) \\
\text { Diff }=\left(0,0, \frac{4}{5}, 0\right)+\frac{2}{3} \Gamma_{4}\end{array}$ & 5 \\
\hline 40 & $\begin{array}{c}z x^{5} y+a z x^{2} y^{5}+b x^{6} y^{3}+ \\
c x^{3} y^{7}+d y^{11}\end{array}$ & $\begin{array}{c}t+z^{3} y+g(z, x, y) \subset \mathbb{P}(33,10,4,3) \\
\mathbb{P}(5,2,3), \text { Diff }=\frac{1}{2} \Gamma+\frac{3}{4} \Gamma_{4}\end{array}$ & 6 \\
\hline 41 & $\begin{array}{c}z x^{5} y+y^{2 n} \\
n=6 ; 7\end{array}$ & $\begin{array}{c}t^{2}+z^{3} y+z x y+y^{2 n} \subset \mathbb{P}(3 n, 2 n-1,4 n-2,3) \\
\text { Diff }=\left(0,0, \frac{4}{5}, 0\right)+\frac{2 n-2}{2 n-1} \Gamma_{4}\end{array}$ & 11,15 \\
\hline
\end{tabular}




\begin{tabular}{|c|c|c|c|}
\hline 42 & $\begin{array}{c}z x^{5} y+a z y^{9}+b x^{5} y^{5}+ \\
c y^{13}\end{array}$ & $\begin{array}{l}t+z^{3} y+g\left(z, x^{1 / 5}, y\right) \subset \mathbb{P}(13,4,8,1) \\
\mathbb{P}(1,2,1), \text { Diff }=\left(0,0, \frac{4}{5}, 0\right)+\frac{1}{2} \Gamma+\frac{7}{8} \Gamma_{4}\end{array}$ & 10 \\
\hline 43 & $\begin{array}{c}z x^{5} y+x y^{2 n} \\
n=4,5 ; 6\end{array}$ & $\begin{array}{c}t^{2}+z^{3} y+g(z, x, y) \subset \mathbb{P}(15 n-1,10 n-5,4 n-2,13) \\
\text { Diff }=\frac{2 n-2}{2 n-1} \Gamma_{4}\end{array}$ & 9,13 \\
\hline 44 & $\begin{array}{l}z x^{5} y+x y^{n} \\
n=9 ; 11 ; 13\end{array}$ & $\begin{aligned} & t+z^{3} y+ g(z, x, y) \subset \mathbb{P}(15 n-2,5 n-5,2 n-2,13) \\
& \mathbb{P}(5,2,13), \text { Diff }=\frac{1}{2} \Gamma+\frac{2 n-3}{2 n-2} \Gamma_{4}\end{aligned}$ & $16,22,26$ \\
\hline 45 & $\begin{array}{l}z x^{5} y+x^{2} y^{n} \\
n=7 ; 9 ; 11\end{array}$ & $\begin{array}{c}t+z^{3} y+g(z, x, y) \subset \mathbb{P}(15 n-4,5 n-5,2 n-2,11) \\
\mathbb{P}(5,2,11), \text { Diff }=\frac{1}{2} \Gamma+\frac{2 n-3}{2 n-2} \Gamma_{4}\end{array}$ & $12,18,22$ \\
\hline 46 & $\begin{array}{l}z x^{5} y+x^{2} y^{2 n} \\
\quad n=4 ; 5\end{array}$ & $\begin{array}{c}t^{2}+z^{3} y+g(z, x, y) \subset \mathbb{P}(15 n-2,10 n-5,4 n-2,11) \\
\text { Diff }=\frac{2 n-2}{2 n-1} \Gamma_{4}\end{array}$ & 7,11 \\
\hline$\overline{47}$ & $\begin{array}{l}z x^{5} y+x^{3} y^{2 n} \\
\quad n=3 ; 4\end{array}$ & $\begin{array}{c}t^{2}+z^{3} y+g(z, x, y) \subset \mathbb{P}(15 n-3,10 n-5,4 n-2,9) \\
\operatorname{Diff}=\frac{2 n-2}{2 n-1} \Gamma_{4}\end{array}$ & 5,9 \\
\hline 48 & $z x^{5} y+x^{3} y^{9}$ & $\begin{array}{c}t+z^{3} y+z x^{5} y+x^{3} y^{9} \subset \mathbb{P}(129,40,16,9) \\
\mathbb{P}(5,2,9), \text { Diff }=\frac{1}{2} \Gamma+\frac{15}{16} \Gamma_{4}\end{array}$ & 18 \\
\hline 49 & $\begin{array}{l}z x^{5} y+x^{4} y^{n} \\
\quad n=5 ; 7\end{array}$ & $\begin{array}{c}t+z^{3} y+g(z, x, y) \subset \mathbb{P}(15 n-8,5 n-5,2 n-2,7) \\
\mathbb{P}(5,2,7), \text { Diff }=\frac{1}{2} \Gamma+\frac{2 n-3}{2 n-2} \Gamma_{4}\end{array}$ & 10,14 \\
\hline 50 & $z x^{5} y+x^{4} y^{6}$ & $\begin{array}{c}t^{2}+z^{3} y+z x^{5} y+x^{4} y^{6} \subset \mathbb{P}(41,25,10,7) \\
\text { Diff }=\frac{4}{5} \Gamma_{4}\end{array}$ & 7 \\
\hline 51 & $\begin{array}{c}z x^{5} y+z y^{n} \\
n=8 ; 10\end{array}$ & $\begin{array}{c}t+z^{3} y+z x y+z y^{n} \subset \mathbb{P}(3 n-1, n-1,2 n-2,2) \\
\mathbb{P}^{2}, \text { Diff }=\left(0,0, \frac{4}{5}, 0\right)+\frac{1}{2} \Gamma+\frac{3}{4} \Gamma_{2}+\frac{2 n-3}{2 n-2} \Gamma_{4}\end{array}$ & 16,20 \\
\hline 52 & $\begin{array}{c}z x^{5} y+z x y^{n} \\
n=6 ; 8\end{array}$ & $\begin{array}{c}t+z^{3} y+g(z, x, y) \subset \mathbb{P}(15 n-7,5 n-5,2 n-2,8) \\
\mathbb{P}(5,1,4), \text { Diff }=\frac{1}{2} \Gamma+\frac{3}{4} \Gamma_{2}+\frac{2 n-3}{2 n-2} \Gamma_{4}\end{array}$ & 12,16 \\
\hline 53 & $z x^{5} y+a z x^{3} y^{4}+b z x y^{7}$ & $\begin{array}{c}t+z^{3} y+g(z, x, y) \subset \mathbb{P}(49,15,6,4) \\
\mathbb{P}(5,1,2), \text { Diff }=\frac{1}{2} \Gamma+\frac{3}{4} \Gamma_{2}+\frac{5}{6} \Gamma_{4}\end{array}$ & 8 \\
\hline 54 & $z x^{5} y+z x^{2} y^{6}$ & $\begin{array}{l}t+z^{3} y+z x^{5} y+z x^{2} y^{6} \subset \mathbb{P}(81,25,10,6) \\
\mathbb{P}(5,1,3), \text { Diff }=\frac{1}{2} \Gamma+\frac{3}{4} \Gamma_{2}+\frac{9}{10} \Gamma_{4}\end{array}$ & 12 \\
\hline
\end{tabular}

5. Singularity $-t^{2}+g_{5}+g(z, x, y)$.

\begin{tabular}{|c|c|c|c|}
\hline 1 & $z^{5}+z x^{i} f_{5-i}(x, y)$ & $\begin{array}{c}t+z^{5}+z x^{i} f_{5-i}(x, y) \subset \mathbb{P}(25,5,4,4) \\
\mathbb{P}(5,1,1), \text { Diff }=\frac{1}{2} \Gamma+\frac{7}{8} \Gamma_{2}\end{array}$ & 8 \\
\hline 2 & $\begin{array}{c}z^{5}+z x^{3} y^{2}+a z^{3} y^{3}+ \\
b z y^{6}\end{array}$ & $\begin{array}{c}t+z^{5}+z x y^{2}+a z^{3} y^{3}+b z y^{6} \subset \mathbb{P}(15,3,8,2) \\
\mathbb{P}(3,4,1), \text { Diff }=\left(0,0, \frac{2}{3}, 0\right)+\frac{1}{2} \Gamma+\frac{3}{4} \Gamma_{2}\end{array}$ & 12 \\
\hline 3 & $z^{5}+z x^{3} y^{2}+z y^{7}$ & $\begin{array}{l}t+z^{5}+z x y^{2}+z y^{7} \subset \mathbb{P}(35,7,20,4) \\
\mathbb{P}(7,5,1), \text { Diff }=\left(0,0, \frac{2}{3}, 0\right)+\frac{1}{2} \Gamma+\frac{7}{8} \Gamma_{2}\end{array}$ & 24 \\
\hline 4 & $z^{5}+z x^{3} y^{2}+z x y^{5}$ & $\begin{array}{c}t+z^{5}+z x^{3} y^{2}+z x y^{5} \subset \mathbb{P}(65,13,12,8) \\
\mathbb{P}(13,3,2), \text { Diff }=\frac{1}{2} \Gamma+\frac{7}{8} \Gamma_{2}\end{array}$ & 16 \\
\hline 5 & $\begin{array}{c}z^{4} x+x^{6}+a z^{2} x y^{3}+ \\
b x y^{6}\end{array}$ & $\begin{array}{c}t^{2}+z^{2} x+x^{6}+a z x y+b x y^{2} \subset \mathbb{P}(6,5,2,5) \\
\text { Diff }=\left(0, \frac{1}{2}, 0, \frac{2}{3}\right)+\frac{4}{5} \Gamma_{3}\end{array}$ & 10 \\
\hline 6 & $z^{4} x+x^{6}+z x y^{4}$ & $\begin{array}{c}t^{2}+z^{4} x+x^{6}+z x y \subset \mathbb{P}(12,5,4,15) \\
\text { Diff }=\left(0,0,0, \frac{3}{4}\right)+\frac{4}{5} \Gamma_{3}\end{array}$ & 5 \\
\hline 7 & $\begin{array}{l}z^{4} x+x^{5} y+z x^{3} y^{2}+ \\
a z^{2} x y^{3}+\overline{b x y^{6}}\end{array}$ & $\begin{array}{c}t+z^{4} x+g(z, x, y) \subset \mathbb{P}(29,6,5,4) \\
\mathbb{P}(3,5,2), \text { Diff }=\frac{1}{2} \Gamma+\frac{3}{4} \Gamma_{3}\end{array}$ & 8 \\
\hline 8 & $\begin{array}{c}z^{4} x+x^{5} y+z x y^{n} \\
n=4 ; 5\end{array}$ & $\begin{array}{c}t+z^{4} x+x^{5} y+z x y^{n} \subset \mathbb{P}(20 n-3,4 n, 4 n-3,12) \\
\mathbb{P}(n, 4 n-3,3), \text { Diff }=\frac{1}{2} \Gamma+\frac{7}{8} \Gamma_{3}\end{array}$ & 10,24 \\
\hline$\overline{9}$ & $z^{4} x+x^{4} y^{2}+a z^{2} x y^{3}+$ & $t^{2}+z^{2} x+x^{4} y^{2}+a z x y^{3}+b x y^{6} \subset \mathbb{P}(11,9,4,3)$ & 6 \\
\hline
\end{tabular}




\begin{tabular}{|c|c|c|c|}
\hline & $b x y^{6}$ & Diff $=\left(0, \frac{1}{2}, 0,0\right)+\frac{2}{3} \Gamma_{3}$ & \\
\hline 10 & $z^{4} x+x^{4} y^{2}+x y^{7}$ & $\begin{array}{c}t^{2}+z x+x^{4} y^{2}+x y^{7} \subset \mathbb{P}(13,21,5,3) \\
\text { Diff }=\left(0, \frac{3}{4}, 0,0\right)+\frac{2}{3} \Gamma_{3}\end{array}$ & 12 \\
\hline 11 & $\begin{array}{c}z^{4} x+x^{4} y^{2}+z x y^{n} \\
n=4 ; 5\end{array}$ & $\begin{array}{c}t^{2}+z^{4} x+g(z, x, y) \subset \mathbb{P}(8 n-3,3 n, 4 n-6,9) \\
\text { Diff }=\frac{2}{3} \Gamma_{3}\end{array}$ & 5,9 \\
\hline 12 & $\begin{array}{c}z^{4} x+z x^{5}+a z^{2} x y^{3}+ \\
b x y^{6}\end{array}$ & $\begin{array}{c}t+z^{4} x+z x^{5}+a z^{2} x y+b x y^{2} \subset \mathbb{P}(19,4,3,8) \\
\mathbb{P}(1,3,2), \text { Diff }=\left(0,0,0, \frac{2}{3}\right)+\frac{1}{2} \Gamma+\frac{7}{8} \Gamma_{3}\end{array}$ & 16 \\
\hline 13 & $\begin{array}{c}z^{4} x+z x^{i} y^{j} f_{5-i-j}(x, y) \\
i \leq 1 \& j \leq 2\end{array}$ & $\begin{array}{c}t+z^{4} x+z x^{i} y^{j} f_{5-i-j}(x, y) \subset \mathbb{P}(19,4,3,3) \\
\mathbb{P}(4,1,1), \text { Diff }=\frac{1}{2} \Gamma+\frac{5}{6} \Gamma_{2}\end{array}$ & 6 \\
\hline 14 & $\begin{array}{c}z^{4} x+z x^{4} y+a z^{2} x y^{3}+ \\
b x y^{6}\end{array}$ & $\begin{array}{c}t+z^{4} x+g(z, x, y) \subset \mathbb{P}(43,9,7,6) \\
\mathbb{P}(3,7,2), \text { Diff }=\frac{1}{2} \Gamma+\frac{5}{6} \Gamma_{3}\end{array}$ & 12 \\
\hline 15 & $z^{4} x+z x^{4} y+z y^{6}$ & $\begin{array}{c}t+z^{4} x+z x^{4} y+z y^{6} \subset \mathbb{P}(91,19,15,12) \\
\mathbb{P}(19,5,4), \text { Diff }=\frac{1}{2} \Gamma+\frac{5}{6} \Gamma_{2}\end{array}$ & 24 \\
\hline 16 & $z^{4} x+z x^{3} y^{2}+x y^{7}$ & $\begin{array}{c}t+z^{4} x+z x^{3} y^{2}+x y^{7} \subset \mathbb{P}(69,14,13,8) \\
\mathbb{P}(7,13,4), \text { Diff }=\frac{1}{2} \Gamma+\frac{3}{4} \Gamma_{3}\end{array}$ & 16 \\
\hline 17 & $z^{4} x+z x^{3} y^{2}+z y^{6}$ & $\begin{array}{c}t^{2}+z^{4} x+z x^{3} y+z y^{3} \subset \mathbb{P}(17,7,6,9) \\
\text { Diff }=\left(0,0,0, \frac{1}{2}\right)+\frac{2}{3} \Gamma_{2}\end{array}$ & 9 \\
\hline 18 & $z^{4} x+z x^{3} y^{2}+z y^{7}$ & $\begin{array}{c}t+z^{4} x+z x^{3} y^{2}+z y^{7} \subset \mathbb{P}(79,16,15,9) \\
\mathbb{P}(16,5,3), \text { Diff }=\frac{1}{2} \Gamma+\frac{3}{4} \Gamma_{2}\end{array}$ & 18 \\
\hline 19 & $z^{4} x+z x^{3} y^{2}+z x y^{5}$ & $\begin{array}{c}t+z^{4} x+z x^{3} y^{2}+z x y^{5} \subset \mathbb{P}(49,10,9,6) \\
\mathbb{P}(5,3,1), \text { Diff }=\frac{1}{2} \Gamma+\frac{5}{6} \Gamma_{2}+\frac{3}{4} \Gamma_{3}\end{array}$ & 12 \\
\hline 20 & $\begin{array}{c}z^{4} y+x^{5} y+a z^{2} y^{4}+ \\
+b y^{7}\end{array}$ & $\begin{array}{c}t^{2}+z^{4} y+x y+a z^{2} y^{4}+b y^{7} \subset \mathbb{P}(7,3,12,2) \\
\text { Diff }=\left(0,0, \frac{4}{5}, 0\right)+\frac{2}{3} \Gamma_{4}\end{array}$ & 5 \\
\hline 21 & $\begin{array}{c}z^{4} y+x^{5} y+y^{2 n} \\
n=4 ; 5\end{array}$ & $\begin{array}{c}t^{2}+z y+x y+y^{2 n} \subset \mathbb{P}(n, 2 n-1,2 n-1,1) \\
\text { Diff }=\left(0, \frac{3}{4}, \frac{4}{5}, 0\right)+\frac{2 n-2}{2 n-1} \Gamma_{4}\end{array}$ & 16,20 \\
\hline 22 & $\begin{array}{c}z^{4} y+x^{5} y+ \\
+a y^{5} f_{2}\left(z, y^{2}\right)\end{array}$ & $\begin{array}{c}t+z^{4} y+x y+a y^{5} f_{2}\left(z, y^{2}\right) \subset \mathbb{P}(9,2,8,1) \\
\mathbb{P}(1,4,1), \text { Diff }=\left(0,0, \frac{4}{5}, 0\right)+\frac{1}{2} \Gamma+\frac{3}{4} \Gamma_{4}\end{array}$ & 10 \\
\hline 23 & $\begin{array}{c}z^{4} y+x^{5} y+x y^{n} \\
n=6 ; 8\end{array}$ & $\begin{array}{c}t^{2}+z^{2} y+g(z, x, y) \subset \mathbb{P}(5 n-1,5 n-5,2 n-2,8) \\
\text { Diff }=\left(0, \frac{1}{2}, 0,0\right)+\frac{n-2}{n-1} \Gamma_{4}\end{array}$ & 12,16 \\
\hline 24 & $\begin{array}{c}z^{4} y+x^{5} y+a x^{3} y^{4}+ \\
+b x y^{7}\end{array}$ & $\begin{array}{c}t^{2}+z^{2} y+g(x, y) \subset \mathbb{P}(17,15,6,4) \\
\text { Diff }=\left(0, \frac{1}{2}, 0,0\right)+\frac{2}{3} \Gamma_{4}\end{array}$ & 8 \\
\hline 25 & $z^{4} y+x^{5} y+x^{2} y^{6}$ & $\begin{array}{c}t^{2}+z y+x^{5} y+x^{2} y^{6} \subset \mathbb{P}(14,25,5,3) \\
\text { Diff }=\left(0, \frac{3}{4}, 0,0\right)+\frac{4}{5} \Gamma_{4}\end{array}$ & 12 \\
\hline 26 & $\begin{array}{c}z^{4} y+x^{5} y+z y^{n} \\
n=5 ; 8\end{array}$ & $\begin{array}{c}t+z^{4} y+x y+z y^{n} \subset \mathbb{P}(4 n-1, n-1,4 n-4,3) \\
\mathbb{P}(1,4,3), \text { Diff }=\left(0,0, \frac{4}{5}, 0\right)+\frac{1}{2} \Gamma+\frac{2 n-3}{2 n-2} \Gamma_{4}\end{array}$ & 10,30 \\
\hline 27 & $\begin{array}{c}z^{4} y+x^{5} y+z x y^{n} \\
n=4 ; 5 ; 6\end{array}$ & $\begin{array}{c}t+z^{4} y+g(z, x, y) \subset \mathbb{P}(20 n-9,5 n-5,4 n-4,11) \\
\mathbb{P}(5,4,11), \text { Diff }=\frac{1}{2} \Gamma+\frac{2 n-3}{2 n-2} \Gamma_{4}\end{array}$ & $6,18,22$ \\
\hline 28 & $\begin{array}{c}z^{4} y+x^{5} y+z x^{2} y^{n} \\
n=3 ; 4\end{array}$ & $\begin{array}{c}t+z^{4} y+g(z, x, y) \subset \mathbb{P}(20 n-13,5 n-5,4 n-4,7) \\
\mathbb{P}(5,4,7), \text { Diff }=\frac{1}{2} \Gamma+\frac{2 n-3}{2 n-2} \Gamma_{4}\end{array}$ & 10,14 \\
\hline 29 & $z^{4} y+z x^{5}+z y^{6}$ & $\begin{array}{c}t+z^{4} y+z x+z y^{6} \subset \mathbb{P}(23,5,18,3) \\
\mathbb{P}(5,6,1), \text { Diff }=\left(0,0, \frac{4}{5}, 0\right)+\frac{1}{2} \Gamma+\frac{5}{6} \Gamma_{2}\end{array}$ & 30 \\
\hline 30 & $\begin{array}{c}z^{4} y+z x^{4} y+a z^{2} y^{4}+ \\
+b y^{7}\end{array}$ & $\begin{array}{c}t^{2}+z^{4} y+z x y+a z^{2} y^{4}+b y^{7} \subset \mathbb{P}(7,3,9,2) \\
\text { Diff }=\left(0,0, \frac{3}{4}, 0\right)+\frac{2}{3} \Gamma_{4}\end{array}$ & 8 \\
\hline 31 & $z^{4} y+z x^{4} y+y^{8}$ & $t^{2}+z^{4} y+z x y+y^{8} \subset \mathbb{P}(16,7,21,4)$ & 16 \\
\hline
\end{tabular}




\begin{tabular}{|c|c|c|c|}
\hline & & Diff $=\left(0,0, \frac{3}{4}, 0\right)+\frac{6}{7} \Gamma_{4}$ & \\
\hline 32 & $\begin{array}{c}z^{4} y+z x^{4} y+x y^{n} \\
n=6 ; 7\end{array}$ & $\begin{array}{c}t+z^{4} y+g(z, x, y) \subset \mathbb{P}(16 n-3,4 n-4,3 n-3,13) \\
\mathbb{P}(4,3,13), \text { Diff }=\frac{1}{2} \Gamma+\frac{2 n-3}{2 n-2} \Gamma_{4}\end{array}$ & 10,26 \\
\hline 33 & $\begin{array}{c}z^{4} y+z x^{4} y+a z^{2} x y^{3}+ \\
+b x^{2} y^{5}\end{array}$ & $\begin{array}{c}t+z^{4} y+g(z, x, y) \subset \mathbb{P}(37,8,6,5) \\
\mathbb{P}(4,3,5), \text { Diff }=\frac{1}{2} \Gamma+\frac{3}{4} \Gamma_{4}\end{array}$ & 10 \\
\hline 34 & $z^{4} y+z x^{4} y+x^{3} y^{4}$ & $\begin{array}{c}t+z^{4} y+z x^{4} y+x^{3} y^{4} \subset \mathbb{P}(55,12,9,7) \\
\mathbb{P}(4,3,7), \text { Diff }=\frac{1}{2} \Gamma+\frac{5}{6} \Gamma_{4}\end{array}$ & 14 \\
\hline 35 & $z^{4} y+z x^{4} y+z y^{6}$ & $\begin{array}{c}t^{2}+z^{4} y+z x^{2} y+z y^{6} \subset \mathbb{P}(23,10,15,6) \\
\text { Diff }=\left(0,0, \frac{1}{2}, 0\right)+\frac{2}{3} \Gamma_{2}+\frac{4}{5} \Gamma_{4}\end{array}$ & 12 \\
\hline 36 & $z^{4} y+z x^{4} y+z x y^{5}$ & $\begin{array}{c}t+z^{4} y+z x^{4} y+z x y^{5} \subset \mathbb{P}(73,16,12,9) \\
\mathbb{P}(4,1,3), \text { Diff }=\frac{1}{2} \Gamma+\frac{5}{6} \Gamma_{2}+\frac{7}{8} \Gamma_{4}\end{array}$ & 18 \\
\hline 37 & $\begin{array}{c}z^{3} x^{2}+z x^{i} y^{j} f_{5-i-j}(x, y) \\
i \leq 1 \& j \leq 2\end{array}$ & $\begin{array}{c}t+z^{3} x^{2}+g(z, x, y) \subset \mathbb{P}(13,3,2,2) \\
\mathbb{P}(3,1,1), \text { Diff }=\frac{1}{2} \Gamma+\frac{3}{4} \Gamma_{2}\end{array}$ & 4 \\
\hline 38 & $\begin{array}{c}z^{3} x y+z x^{i}(x+y)^{j} f_{5-i-j} \\
(x, y), i \leq 1 \& j \leq 2\end{array}$ & $\begin{array}{c}t+z^{3} x^{2}+g(z, x, y) \subset \mathbb{P}(13,3,2,2) \\
\mathbb{P}(3,1,1), \text { Diff }=\frac{1}{2} \Gamma+\frac{3}{4} \Gamma_{2}\end{array}$ & 4 \\
\hline 39 & $z^{3} x^{2}+z x^{5}+z x y^{5}$ & $\begin{array}{l}t+z^{3} x^{2}+z x^{5}+z x y \subset \mathbb{P}(13,3,2,8) \\
\mathbb{P}(3,1,4), \text { Diff }=\left(0,0,0, \frac{4}{5}\right)+\frac{1}{2} \Gamma+\frac{3}{4} \Gamma_{2}\end{array}$ & 16 \\
\hline 40 & $z^{3} x^{2}+z x^{4} y+z x y^{5}$ & $\begin{array}{c}t+z^{3} x^{2}+z x^{4} y+z x y^{5} \subset \mathbb{P}(49,11,8,6) \\
\mathbb{P}(11,4,3), \text { Diff }=\frac{1}{2} \Gamma+\frac{3}{4} \Gamma_{2}\end{array}$ & 12 \\
\hline 41 & $z^{3} x^{2}+z x^{3} y^{2}+z y^{7}$ & $\begin{array}{c}t+z^{3} x^{2}+z x^{3} y^{2}+z y^{7} \subset \mathbb{P}(53,11,10,6) \\
\mathbb{P}(11,5,3), \text { Diff }=\frac{1}{2} \Gamma+\frac{3}{4} \Gamma_{2}\end{array}$ & 12 \\
\hline 42 & $\begin{array}{c}z^{3} x^{2}+z x^{3} y^{2}+a z^{3} y^{3}+ \\
+b z x y^{5}\end{array}$ & $\begin{array}{c}t+z^{3} x^{2}+z x^{3} y^{2}+a z^{3} y^{3}+z x y^{5} \subset \mathbb{P}(33,7,6,4) \\
\mathbb{P}(7,3,2), \text { Diff }=\frac{1}{2} \Gamma+\frac{3}{4} \Gamma_{2}\end{array}$ & 8 \\
\hline 43 & $\begin{array}{c}z^{3} y^{2}+z x^{5}+z y^{n} \\
n=7 ; 9\end{array}$ & $\begin{array}{c}t+z^{3} y^{2}+z x+z y^{n} \subset \mathbb{P}(3 n-2, n-2,2 n, 2) \\
\mathbb{P}(n-2, n, 1), \text { Diff }=\left(0,0, \frac{4}{5}, 0\right)+\frac{1}{2} \Gamma+\frac{3}{4} \Gamma_{2}\end{array}$ & 16,20 \\
\hline 44 & $\begin{array}{c}z^{3} y^{2}+z x^{5}+z x y^{n} \\
n=5\end{array}$ & $\begin{array}{c}t+z^{3} y^{2}+g(z, x, y) \subset \mathbb{P}(15 n-8,5 n-8,2 n, 8) \\
\mathbb{P}(5 n-8, n, 4), \text { Diff }=\frac{1}{2} \Gamma+\frac{3}{4} \Gamma_{2}\end{array}$ & 12,16 \\
\hline 45 & $\begin{aligned} z^{3} y^{2}+ & z x^{5}+a z x^{3} y^{3}+ \\
& +b z x y^{6}\end{aligned}$ & $\begin{array}{c}t+z^{3} y^{2}+g(z, x, y) \subset \mathbb{P}(41,11,6,4) \\
\mathbb{P}(11,3,2), \text { Diff }=\frac{1}{2} \Gamma+\frac{3}{4} \Gamma_{2}\end{array}$ & 8 \\
\hline 46 & $z^{3} y^{2}+z x^{5}+z x^{2} y^{5}$ & $\begin{array}{c}t+z^{3} y^{2}+z x^{5}+z x^{2} y^{5} \subset \mathbb{P}(69,19,10,6) \\
\mathbb{P}(19,5,3), \text { Diff }=\frac{1}{2} \Gamma+\frac{3}{4} \Gamma_{2}\end{array}$ & 12 \\
\hline 47 & $\begin{array}{c}z^{3} x y+x^{5}+x y^{n} \\
n=5 ; 9,11 ; 15\end{array}$ & $\begin{array}{l}t+z x y+x^{5}+x y^{n} \subset \mathbb{P}(5 n, 4 n-4, n, 4) \\
\mathbb{P}(n-1, n, 1), \text { Diff }=\left(0, \frac{2}{3}, 0,0\right)+\frac{1}{2} \Gamma+\frac{7}{8} \Gamma_{3}\end{array}$ & $8,16,24$ \\
\hline 48 & $\begin{array}{l}z^{5}+z x^{3} y+a z x^{2} y^{n+1}+ \\
\quad+c z y^{3 n+1}, n=2,4\end{array}$ & $\begin{array}{c}t+g_{5}+g(z, x, y) \subset \mathbb{P}(15 n+5,3 n+1,4 n, 4) \\
\mathbb{P}(3 n+1, n, 1), \text { Diff }=\frac{1}{2} \Gamma+\frac{7}{8} \Gamma_{2}\end{array}$ & 8 \\
\hline 49 & $\begin{array}{c}z^{3} x y+x^{5}+a z x^{3} y^{2}+ \\
b z x y^{7}+c x^{3} y^{5}+d x y^{10}\end{array}$ & $\begin{array}{c}t+z^{3} x y+g(z, x, y) \subset \mathbb{P}(25,6,5,2) \\
\mathbb{P}(3,5,1), \text { Diff }=\frac{1}{2} \Gamma+\frac{3}{4} \Gamma_{3}\end{array}$ & 4 \\
\hline 50 & $\begin{array}{c}z^{3} x y+x^{5}+a z x y^{9}+ \\
b x y^{13}\end{array}$ & $\begin{array}{c}t+z^{3} x y+g(z, x, y) \subset \mathbb{P}(65,16,13,4) \\
\mathbb{P}(4,13,1), \text { Diff }=\frac{1}{2} \Gamma+\frac{7}{8} \Gamma_{3}\end{array}$ & 8 \\
\hline 51 & $\begin{array}{l}z^{3} x y+x^{5}+z x y^{n} \\
n=4,6 ; 8,10\end{array}$ & $\begin{array}{c}t+z^{3} x y+g(z, x, y) \subset \mathbb{P}(15 n-5,4 n-4,3 n-1,8) \\
\mathbb{P}(n-1,3 n-1,2), \text { Diff }=\frac{1}{2} \Gamma+\frac{7}{8} \Gamma_{3}\end{array}$ & 8,16 \\
\hline 52 & $\begin{array}{c}z^{3} x y+x^{6}+x y^{n} \\
n=6 ; 8 ; 9\end{array}$ & $\begin{array}{c}t^{2}+z x y+g(x, y) \subset \mathbb{P}(3 n, 5 n-5, n, 5) \\
\operatorname{Diff}=\left(0, \frac{2}{3}, 0,0\right)+\frac{4}{5} \Gamma_{3}\end{array}$ & $9,12,15$ \\
\hline 53 & $z^{3} x y+x^{6}+a z x y^{5}+$ & $t^{2}+z^{3} x y+g(z, x, y) \subset \mathbb{P}(21,10,7,5)$ & 5 \\
\hline
\end{tabular}




\begin{tabular}{|c|c|c|c|}
\hline & $b x y^{7}$ & Diff $=\frac{4}{5} \Gamma_{3}$ & \\
\hline 54 & $\begin{array}{c}z^{3} x y+x^{6}+z x y^{n} \\
n=4 ; 6\end{array}$ & $\begin{array}{c}t^{2}+z^{3} x y+g(z, x, y) \subset \mathbb{P}(9 n-3,5 n-5,3 n-1,10) \\
\text { Diff }=\frac{4}{5} \Gamma_{3}\end{array}$ & 5,10 \\
\hline 55 & $\begin{array}{c}z^{3} x y+x^{5} y+a z x^{2} y^{n+1}+ \\
b y^{3 n+1}, n=2 b \neq 0 ; 4\end{array}$ & $\begin{array}{c}t+z^{3} x y+g(z, x, y) \subset \mathbb{P}(15 n+5,4 n, 3 n, 5) \\
\mathbb{P}(4,3,5), \text { Diff }=\frac{1}{2} \Gamma+\frac{2 n-1}{2 n} \Gamma_{4}\end{array}$ & 4,10 \\
\hline 56 & $\begin{array}{c}z^{3} x y+x^{5} y+y^{2 n} \\
n=4 ; 6,7\end{array}$ & $\begin{array}{c}t^{2}+z x y+g(x, y) \subset \mathbb{P}(5 n, 8 n-4,2 n-1,5) \\
\text { Diff }=\left(0, \frac{2}{3}, 0,0\right)+\frac{2 n-2}{2 n-1} \Gamma_{4}\end{array}$ & 7,15 \\
\hline 57 & $\begin{array}{c}z^{3} x y+x^{5} y+y^{n} \\
n=9 ; 15\end{array}$ & $\begin{array}{l}t+z x y+g(z, x, y) \subset \mathbb{P}(5 n, 4 n-4, n-1,5) \\
\mathbb{P}(4,1,5), \text { Diff }=\left(0, \frac{2}{3}, 0,0\right)+\frac{1}{2} \Gamma+\frac{2 n-3}{2 n-2} \Gamma_{4}\end{array}$ & 16,30 \\
\hline 58 & $\begin{array}{c}z^{3} x y+x^{5} y+a z x^{2} y^{4}+ \\
+b y^{10}\end{array}$ & $\begin{array}{c}t^{2}+z^{3} x y+g(z, x, y) \subset \mathbb{P}(25,12,9,5) \\
\text { Diff }=\frac{2}{3} \Gamma_{4}\end{array}$ & 5 \\
\hline 59 & $z^{3} x y+y x^{i} f_{5-i}\left(x, y^{2}\right)$ & $\begin{array}{c}t+z x y+g(z, x, y) \subset \mathbb{P}(11,8,2,1) \\
\mathbb{P}(4,1,1), \text { Diff }=\left(0, \frac{2}{3}, 0,0\right)+\frac{1}{2} \Gamma+\frac{3}{4} \Gamma_{4} \\
\end{array}$ & 6 \\
\hline 60 & $\begin{array}{c}z^{3} x y+x^{5} y+x y^{n} \\
\quad n=6 ; 8 ; 12\end{array}$ & $\begin{array}{c}t+z x y+g(z, x, y) \subset \mathbb{P}(5 n-1,4 n-4, n-1,4) \\
\mathbb{P}^{2}, \text { Diff }=\left(0, \frac{2}{3}, 0,0\right)+\frac{1}{2} \Gamma+\frac{7}{8} \Gamma_{3}+\frac{2 n-3}{2 n-2} \Gamma_{4}\end{array}$ & $10,16,24$ \\
\hline 61 & $\begin{array}{c}z^{3} x y+x^{5} y+a z^{2} x^{3}+ \\
b z x^{3} y^{2}+c z x y^{5}+d x^{3} y^{4} \\
\quad+e x y^{7},|e|+|c| \neq 0\end{array}$ & $\begin{array}{c}t+z^{3} x y+g(z, x, y) \subset \mathbb{P}(17,4,3,2) \\
\mathbb{P}(2,3,1), \text { Diff }=\frac{1}{2} \Gamma+\frac{3}{4} \Gamma_{3}\end{array}$ & 4 \\
\hline 62 & $\begin{aligned} z^{3} x y+ & x^{5} y+a z x y^{7}+ \\
& +b x y^{10}\end{aligned}$ & $\begin{array}{l}t+z^{3} x y+g(z, x, y) \subset \mathbb{P}(49,12,9,4) \\
\mathbb{P}(1,3,1), \text { Diff }=\frac{1}{2} \Gamma+\frac{7}{8} \Gamma_{3}+\frac{5}{6} \Gamma_{4}\end{array}$ & 8 \\
\hline 63 & $\begin{array}{c}z^{3} x y+x^{5} y+a x^{3} y^{6}+ \\
b x y^{11}\end{array}$ & $\begin{array}{c}t+z x y+g(z, x, y) \subset \mathbb{P}(27,20,5,2) \\
\mathbb{P}(2,1,1), \text { Diff }=\left(0, \frac{2}{3}, 0,0\right)+\frac{1}{2} \Gamma+\frac{3}{4} \Gamma_{3}+\frac{9}{10} \Gamma_{4}\end{array}$ & 12 \\
\hline 64 & $\begin{array}{c}z^{3} x y+x^{5} y+x^{2} y^{n} \\
n=5 ; 9\end{array}$ & $\begin{array}{c}t+z x y+g(z, x, y) \subset \mathbb{P}(5 n-2,4 n-4, n-1,3) \\
\mathbb{P}(4,1,3), \text { Diff }=\left(0, \frac{2}{3}, 0,0\right)+\frac{1}{2} \Gamma+\frac{2 n-3}{2 n-2} \Gamma_{4}\end{array}$ & 10,18 \\
\hline 65 & $\begin{array}{l}z^{3} x y+x^{5} y+x^{2} y^{2 n} \\
n=3 ; 4\end{array}$ & $\begin{array}{c}t^{2}+z x y+g(z, x, y) \subset \mathbb{P}(5 n-1,8 n-4,2 n-1,3) \\
\operatorname{Diff}=\left(0, \frac{2}{3}, 0,0\right)+\frac{2 n-2}{2 n-1} \Gamma_{4}\end{array}$ & 9 \\
\hline 66 & $\begin{array}{l}z^{3} x y+x^{5} y+z y^{n} \\
\quad n=5 ; 7 ; 9 ; 11\end{array}$ & $\begin{array}{c}t+z^{3} x y+g(z, x, y) \subset \mathbb{P}(15 n-4,4 n-4,3 n-3,11) \\
\mathbb{P}(4,3,11), \text { Diff }=\frac{1}{2} \Gamma+\frac{2 n-3}{2 n-2} \Gamma_{4}\end{array}$ & $\begin{array}{l}8,14,16 \\
22\end{array}$ \\
\hline 67 & $\begin{array}{c}z^{3} x y+x^{5} y+z y^{2 n} \\
n=3 ; 4,5\end{array}$ & $\begin{array}{c}t^{2}+z^{3} x y+g(z, x, y) \subset \mathbb{P}(15 n-2,8 n-4,6 n-3,11) \\
\text { Diff }=\frac{2 n-2}{2 n-1} \Gamma_{4}\end{array}$ & 5,11 \\
\hline 68 & $\begin{array}{c}z^{3} x y+x^{5} y+z x y^{n} \\
n=4 ; 6 ; 8\end{array}$ & $\begin{array}{c}t+z^{3} x y+g(z, x, y) \subset \mathbb{P}(15 n-7,4 n-4,3 n-3,8) \\
\mathbb{P}(1,3,2), \text { Diff }=\frac{1}{2} \Gamma+\frac{7}{8} \Gamma_{3}+\frac{2 n-3}{2 n-2} \Gamma_{4}\end{array}$ & $8,10,16$ \\
\hline 69 & $\begin{array}{c}z^{3} x y+x^{5} y+z^{2} y^{2 n} \\
n=2 ; 3\end{array}$ & $\begin{array}{c}t^{2}+z^{3} x y+g(z, x, y) \subset \mathbb{P}(15 n-4,8 n-4,6 n-3,7) \\
\text { Diff }=\frac{2 n-2}{2 n-1} \Gamma_{4}\end{array}$ & 6,7 \\
\hline 70 & $\begin{array}{c}z^{3} x y+x^{5} y+z^{2} y^{n} \\
n=5 ; 7\end{array}$ & $\begin{array}{c}t+z^{3} x y+g(z, x, y) \subset \mathbb{P}(15 n-8,4 n-4,3 n-3,7) \\
\mathbb{P}(4,3,7), \text { Diff }=\frac{1}{2} \Gamma+\frac{2 n-3}{2 n-2} \Gamma_{4}\end{array}$ & 8,14 \\
\hline 71 & $\begin{array}{l}z^{3} x y+x^{7}+a z x y^{5}+ \\
b x y^{7}\end{array}$ & $\begin{array}{c}t+z^{3} x y+g(z, x, y) \subset \mathbb{P}(49,12,7,6) \\
\mathbb{P}(2,7,1), \text { Diff }=\frac{1}{2} \Gamma+\frac{11}{12} \Gamma_{4}\end{array}$ & 12 \\
\hline 72 & $z^{3} x y+x^{7}+x^{3} y^{5}$ & $\begin{array}{l}t+z x y+x^{7}+x^{3} y^{5} \subset \mathbb{P}(35,26,5,4) \\
\mathbb{P}(13,5,2), \text { Diff }=\left(0, \frac{2}{3}, 0,0\right)+\frac{1}{2} \Gamma+\frac{3}{4} \Gamma_{3}\end{array}$ & 24 \\
\hline 73 & $z^{3} x y+x^{6} y+y^{8}$ & $\begin{array}{c}t^{2}+z x y+x^{6} y+y^{8} \subset \mathbb{P}(24,35,7,6) \\
\text { Diff }=\left(0, \frac{2}{3}, 0,0\right)+\frac{6}{7} \Gamma_{4}\end{array}$ & 9 \\
\hline 74 & $\begin{array}{c}z^{3} x y+x^{6} y+a x^{3} y^{5}+ \\
+b y^{9}\end{array}$ & $\begin{array}{c}t+z x y+x^{6} y+a x^{3} y^{5}+b y^{9} \subset \mathbb{P}(27,20,4,3) \\
\mathbb{P}(5,1,3), \text { Diff }=\left(0, \frac{2}{3}, 0,0\right)+\frac{1}{2} \Gamma+\frac{7}{8} \Gamma_{4}\end{array}$ & 18 \\
\hline
\end{tabular}




\begin{tabular}{|c|c|c|c|}
\hline 75 & $\begin{array}{c}z^{3} x y+x^{6} y+a z x y^{5}+ \\
b x y^{7}\end{array}$ & $\begin{array}{c}t+z^{3} x y+g(z, x, y) \subset \mathbb{P}(41,10,6,5) \\
\mathbb{P}(1,3,1), \text { Diff }=\frac{1}{2} \Gamma+\frac{9}{10} \Gamma_{3}+\frac{3}{4} \Gamma_{4}\end{array}$ & 10 \\
\hline 76 & $z^{3} x y+x^{6} y+x y^{8}$ & $\begin{array}{l}t+z x y+x^{6} y+x y^{8} \subset \mathbb{P}(47,35,7,5) \\
\mathbb{P}^{2}, \text { Diff }=\left(0, \frac{2}{3}, 0,0\right)+\frac{1}{2} \Gamma+\frac{9}{10} \Gamma_{3}+\frac{13}{14} \Gamma_{4}\end{array}$ & 30 \\
\hline 77 & $z^{3} x y+x^{6} y+x^{2} y^{6}$ & $\begin{array}{c}t^{2}+z x y+x^{6} y+x^{2} y^{6} \subset \mathbb{P}(17,25,5,4) \\
\operatorname{Diff}=\left(0, \frac{2}{3}, 0,0\right)+\frac{4}{5} \Gamma_{4}\end{array}$ & 12 \\
\hline 78 & $\begin{array}{c}z^{3} x y+x^{6} y+z y^{n} \\
n=6 ; 7\end{array}$ & $\begin{array}{c}t+z^{3} x y+g(z, x, y) \subset \mathbb{P}(18 n-5,5 n-5,3 n-3,13) \\
\mathbb{P}(5,3,13), \text { Diff }=\frac{1}{2} \Gamma+\frac{2 n-3}{2 n-2} \Gamma_{4}\end{array}$ & 10,26 \\
\hline 79 & $z^{3} x y+x^{6} y+z x^{2} y^{4}$ & $\begin{array}{c}t+z^{3} x y+x^{6} y+z x^{2} y^{4} \subset \mathbb{P}(61,15,9,7) \\
\mathbb{P}(5,3,7), \text { Diff }=\frac{1}{2} \Gamma+\frac{2}{3} \Gamma_{4}\end{array}$ & 14 \\
\hline 80 & $z^{3} x y+x^{6} y+z^{2} y^{4}$ & $\begin{array}{c}t^{2}+z^{3} x y+x^{6} y+z^{2} y^{4} \subset \mathbb{P}(31,15,9,8) \\
\text { Diff }=\frac{2}{3} \Gamma_{4}\end{array}$ & 8 \\
\hline 81 & $\begin{array}{l}z^{3} x y+z x^{5}+x^{5} y^{2}+ \\
\quad+a z x y^{5}+b x y^{7}\end{array}$ & $\begin{array}{c}t+z^{3} x y+g(z, x, y) \subset \mathbb{P}(33,8,5,4) \\
\mathbb{P}(2,5,1), \text { Diff }=\frac{1}{2} \Gamma+\frac{7}{8} \Gamma_{3}\end{array}$ & 8 \\
\hline 82 & $\begin{aligned} z^{3} x y+ & x^{5} y^{2}+a x^{3} y^{5}+ \\
& +b x y^{8}\end{aligned}$ & $\begin{array}{c}t+z x y+g(x, y) \subset \mathbb{P}(19,14,3,2) \\
\mathbb{P}(7,3,1), \text { Diff }=\left(0, \frac{2}{3}, 0,0\right)+\frac{1}{2} \Gamma+\frac{3}{4} \Gamma_{3}\end{array}$ & 12 \\
\hline 83 & $z^{3} x y+x^{5} y^{2}+x y^{9}$ & $\begin{array}{c}t+z x y+x^{5} y^{2}+x y^{9} \subset \mathbb{P}(43,32,7,4) \\
\mathbb{P}(8,7,1), \text { Diff }=\left(0, \frac{2}{3}, 0,0\right)+\frac{1}{2} \Gamma+\frac{7}{8} \Gamma_{3}\end{array}$ & 24 \\
\hline 84 & $z^{3} x y+x^{5} y^{2}+z x y^{6}$ & $\begin{array}{c}t+z^{3} x y+x^{5} y^{2}+z x y^{6} \subset \mathbb{P}(81,20,13,8) \\
\mathbb{P}(5,13,2), \text { Diff }=\frac{1}{2} \Gamma+\frac{7}{8} \Gamma_{3}\end{array}$ & 16 \\
\hline 85 & $\begin{array}{c}z^{3} x y+z x^{5}+x y^{n} \\
n=6 ; 8\end{array}$ & $\begin{array}{c}t+z^{3} x y+g(z, x, y) \subset \mathbb{P}(14 n+1,4 n-4,2 n+1,12) \\
\mathbb{P}(n-1,2 n+1,3), \text { Diff }=\frac{1}{2} \Gamma+\frac{7}{8} \Gamma_{3}\end{array}$ & 8,24 \\
\hline 86 & $z^{3} x y+z x^{5}+x^{3} y^{5}$ & $\begin{array}{c}t+z^{3} x y+z x^{5}+x^{3} y^{5} \subset \mathbb{P}(73,18,11,8) \\
\mathbb{P}(9,11,4), \text { Diff }=\frac{1}{2} \Gamma+\frac{3}{4} \Gamma_{3}\end{array}$ & 16 \\
\hline 87 & $\begin{array}{c}z^{3} x y+z x^{5}+z y^{n} \\
n=6 ; 7\end{array}$ & $\begin{array}{c}t+z^{3} x y+g(z, x, y) \subset \mathbb{P}(14 n-5,4 n-5,2 n, 10) \\
\mathbb{P}(4 n-5, n, 5), \text { Diff }=\frac{1}{2} \Gamma+\frac{3}{4} \Gamma_{2}\end{array}$ & 8,20 \\
\hline 88 & $z^{3} x y+z x^{5}+z x^{2} y^{4}$ & $\begin{array}{c}t+z^{3} x y+z x^{5}+z x^{2} y^{4} \subset \mathbb{P}(53,13,8,6) \\
\mathbb{P}(13,4,3), \text { Diff }=\frac{1}{2} \Gamma+\frac{3}{4} \Gamma_{2}\end{array}$ & 12 \\
\hline 89 & $\begin{array}{c}z^{3} x y+z x^{4} y+y^{n} \\
\quad n=7 ; 9 ; 11\end{array}$ & $\begin{array}{c}t+z^{3} x y+g(z, x, y) \subset \mathbb{P}(11 n, 3 n-3,2 n-2,11) \\
\mathbb{P}(3,2,11), \text { Diff }=\frac{1}{2} \Gamma+\frac{2 n-3}{2 n-2} \Gamma_{4}\end{array}$ & $12,16,22$ \\
\hline 90 & $\begin{array}{c}z^{3} x y+z x^{4} y+y^{2 n} \\
n=4 ; 5\end{array}$ & $\begin{array}{c}t^{2}+z^{3} x y+g(z, x, y) \subset \mathbb{P}(11 n, 6 n-3,4 n-2,11) \\
\text { Diff }=\frac{2 n-2}{2 n-1} \Gamma_{4}\end{array}$ & 7,11 \\
\hline 91 & $\begin{array}{c}z^{3} x y+z x^{4} y+x y^{2 n} \\
n=3 ; 4\end{array}$ & $\begin{array}{c}t^{2}+z^{3} x y+g(z, x, y) \subset \mathbb{P}(11 n-1,6 n-3,4 n-2,9) \\
\text { Diff }=\frac{2}{3} \Gamma_{3}+\frac{2 n-2}{2 n-1} \Gamma_{4}\end{array}$ & 5,9 \\
\hline 92 & $\begin{array}{c}z^{3} x y+z x^{4} y+a z x y^{5}+ \\
b x^{4} y^{3}+c x y^{7}\end{array}$ & $\begin{array}{l}t+z^{3} x y+g(z, x, y) \subset \mathbb{P}(25,6,4,3) \\
\mathbb{P}(1,2,1), \text { Diff }=\frac{1}{2} \Gamma+\frac{5}{6} \Gamma_{3}+\frac{3}{4} \Gamma_{4}\end{array}$ & 6 \\
\hline 93 & $z^{3} x y+z x^{4} y+x y^{9}$ & $\begin{array}{c}t+z^{3} x y+z x^{4} y+x y^{9} \subset \mathbb{P}(97,24,16,9) \\
\mathbb{P}(1,2,3), \text { Diff }=\frac{1}{2} \Gamma+\frac{5}{6} \Gamma_{3}+\frac{15}{16} \Gamma_{4}\end{array}$ & 18 \\
\hline 94 & $\begin{array}{c}z^{3} x y+z x^{4} y+x^{2} y^{n} \\
n=5 ; 7\end{array}$ & $\begin{array}{c}t+z^{3} x y+g(z, x, y) \subset \mathbb{P}(11 n-4,3 n-3,2 n-2,7) \\
\mathbb{P}(3,2,7), \text { Diff }=\frac{1}{2} \Gamma+\frac{2 n-3}{2 n-2} \Gamma_{4}\end{array}$ & 8,14 \\
\hline 95 & $z^{3} x y+z x^{4} y+x^{2} y^{6}$ & $\begin{array}{c}t^{2}+z^{3} x y+z x^{4} y+x^{2} y^{6} \subset \mathbb{P}(31,15,10,7) \\
\text { Diff }=\frac{4}{5} \Gamma_{4}\end{array}$ & 7 \\
\hline 96 & $\begin{array}{c}z^{3} x y+z x^{4} y+a z^{2} y^{4}+ \\
b x^{3} y^{4}, a \neq 0\end{array}$ & $\begin{array}{c}t^{2}+z^{3} x y+z x^{4} y+a z^{2} y^{4}+b x^{3} y^{4} \subset \mathbb{P}(19,9,6,5) \\
\text { Diff }=\frac{2}{3} \Gamma_{4}\end{array}$ & 5 \\
\hline
\end{tabular}




\begin{tabular}{|c|c|c|c|}
\hline 97 & $\begin{array}{c}z^{3} x y+z x^{4} y+a z^{2} y^{5}+ \\
b x^{3} y^{5}\end{array}$ & $\begin{array}{c}t+z^{3} x y+z x^{4} y+a z^{2} y^{5}+b x^{3} y^{5} \subset \mathbb{P}(49,12,8,5) \\
\mathbb{P}(3,2,5), \text { Diff }=\frac{1}{2} \Gamma+\frac{7}{8} \Gamma_{4}\end{array}$ & 10 \\
\hline 98 & $\begin{array}{c}z^{3} x y+z x^{4} y+z y^{n} \\
n=6 ; 8\end{array}$ & $\begin{array}{c}t+z^{3} x y+g(z, x, y) \subset \mathbb{P}(11 n-3,3 n-3,2 n-2,8) \\
\mathbb{P}(3,1,4), \text { Diff }=\frac{1}{2} \Gamma+\frac{3}{4} \Gamma_{2}+\frac{2 n-3}{2 n-2} \Gamma_{4}\end{array}$ & 10,16 \\
\hline 99 & $z^{3} x y+z x^{4} y+z y^{7}$ & $\begin{array}{c}t+z^{3} x y+z x^{4} y+z y^{7} \subset \mathbb{P}(37,9,6,4) \\
\mathbb{P}(3,1,2), \text { Diff }=\frac{1}{2} \Gamma+\frac{3}{4} \Gamma_{2}+\frac{5}{6} \Gamma_{4}\end{array}$ & 8 \\
\hline 100 & $z^{3} x y+z x^{4} y+z x y^{6}$ & $\begin{array}{c}t+z^{3} x y+z x^{4} y+z x y^{6} \subset \mathbb{P}(61,15,10,6) \\
\mathbb{P}^{2}, \text { Diff }=\frac{1}{2} \Gamma+\frac{3}{4} \Gamma_{2}+\frac{5}{6} \Gamma_{3}+\frac{9}{10} \Gamma_{4}\end{array}$ & 12 \\
\hline 101 & $\begin{array}{l}z^{4} x+z x^{3} y+x y^{n} \\
\quad n=5 ; 7 ; 9 ; 11\end{array}$ & $\begin{array}{c}t+g_{5}+g(z, x, y) \subset \mathbb{P}(11 n-4,2 n, 3 n-4,8) \\
\mathbb{P}(n, 3 n-4,4), \text { Diff }=\frac{1}{2} \Gamma+\frac{3}{4} \Gamma_{3}\end{array}$ & $\begin{array}{c}6,10,12 \\
16 \\
\end{array}$ \\
\hline 102 & $\begin{array}{c}z^{4} x+z x^{3} y+a z^{2} x y^{n}+ \\
b x y^{2 n}, n=3 b \neq 0 ; 5\end{array}$ & $\begin{array}{l}t+g_{5}+g(z, x, y) \subset \mathbb{P}(11 n-2,2 n, 3 n-2,4) \\
\mathbb{P}(n, 3 n-2,2), \text { Diff }=\frac{1}{2} \Gamma+\frac{3}{4} \Gamma_{3}\end{array}$ & 4,8 \\
\hline 103 & $\begin{array}{c}z^{4} x+z x^{3} y+a z^{2} x y^{4}+ \\
b z x y^{6}+c x^{3} y^{3}+d x y^{8}\end{array}$ & $\begin{array}{c}t+g_{5}+g(z, x, y) \subset \mathbb{P}(21,4,5,2) \\
\mathbb{P}(2,5,1), \text { Diff }=\frac{1}{2} \Gamma+\frac{3}{4} \Gamma_{3}\end{array}$ & 4 \\
\hline 104 & $\begin{array}{c}z^{4} x+z x^{3} y+z y^{2 n+1} \\
n=2 ; 4,5\end{array}$ & $\begin{array}{c}t^{2}+g_{5}+g(z, x, y) \subset \mathbb{P}(11 n+6,4 n+3,6 n, 9) \\
\operatorname{Diff}=\frac{2}{3} \Gamma_{2}\end{array}$ & 3,9 \\
\hline 105 & $\begin{array}{c}z^{4} x+z x^{3} y+z y^{n} \\
n=6 ; 8 ; 12\end{array}$ & $\begin{aligned} t+g_{5}+g(z, x, y) \subset \mathbb{P}(11 n+1,2 n+1,3 n-3,9) \\
\mathbb{P}(2 n+1, n-1,3), \text { Diff }=\frac{1}{2} \Gamma+\frac{5}{6} \Gamma_{2}\end{aligned}$ & $8,12,18$ \\
\hline 106 & $\begin{array}{c}z^{4} x+z x^{3} y+a z x^{2} y^{3}+ \\
b z x y^{5}+c z y^{7},|b|+|c| \neq 0\end{array}$ & $\begin{array}{c}t^{2}+g_{5}+g(z, x, y) \subset \mathbb{P}(13,5,6,3) \\
\text { Diff }=\frac{2}{3} \Gamma_{2}\end{array}$ & 3 \\
\hline 107 & $\begin{array}{c}z^{4} x+z x^{3} y+a z x^{2} y^{4}+ \\
b z x y^{7}+c z y^{10}\end{array}$ & $\begin{array}{c}t+g_{5}+g(z, x, y) \subset \mathbb{P}(37,7,9,3) \\
\mathbb{P}(7,3,1), \text { Diff }=\frac{1}{2} \Gamma+\frac{5}{6} \Gamma_{2}\end{array}$ & 6 \\
\hline 108 & $\begin{array}{c}z^{4} x+z x^{3} y+z x y^{n} \\
n=4 ; 8\end{array}$ & $\begin{array}{c}t+g_{5}+g(z, x, y) \subset \mathbb{P}(11 n-3,2 n, 3 n-3,6) \\
\mathbb{P}(n, n-1,1), \text { Diff }=\frac{1}{2} \Gamma+\frac{5}{6} \Gamma_{2}+\frac{3}{4} \Gamma_{3}\end{array}$ & 6,12 \\
\hline 109 & $\begin{array}{c}z^{4} x+x^{4} y+x y^{n} \\
n=5 ; 9\end{array}$ & $\begin{array}{c}t^{2}+z^{2} x+x^{4} y+x y^{n} \subset \mathbb{P}(4 n-1,3 n, 2 n-2,6) \\
\text { Diff }=\left(0, \frac{1}{2}, 0,0\right)+\frac{2}{3} \Gamma_{3}\end{array}$ & 3,12 \\
\hline 110 & $\begin{array}{c}z^{4} x+x^{4} y+a z^{2} x y^{3}+ \\
+b x y^{6}\end{array}$ & $\begin{array}{c}t^{2}+g_{5}+g(z, x, y) \subset \mathbb{P}(23,9,10,6) \\
\text { Diff }=\frac{2}{3} \Gamma_{3}\end{array}$ & 3 \\
\hline 111 & $\begin{array}{c}z^{4} x+x^{4} y+a z^{2} x y^{4}+ \\
b z x y^{6}+c x y^{8}\end{array}$ & $\begin{array}{c}t+g_{5}+g(z, x, y) \subset \mathbb{P}(31,6,7,3) \\
\mathbb{P}(2,7,1), \text { Diff }=\frac{1}{2} \Gamma+\frac{5}{6} \Gamma_{3}\end{array}$ & 6 \\
\hline 112 & $\begin{array}{c}z^{4} x+x^{4} y+z x y^{n} \\
n=4,5 ; 7\end{array}$ & $\begin{array}{c}t+g_{5}+g(z, x, y) \subset \mathbb{P}(16 n-3,3 n, 4 n-3,9) \\
\mathbb{P}(n, 4 n-3,3), \text { Diff }=\frac{1}{2} \Gamma+\frac{5}{6} \Gamma_{3}\end{array}$ & 6,18 \\
\hline 113 & $\begin{array}{c}z^{3} x^{2}+z x^{3} y+z y^{n} \\
n=5 ; 9\end{array}$ & $\begin{array}{c}t+g_{5}+g(z, x, y) \subset \mathbb{P}(7 n+2, n+2,2 n-2,6) \\
\mathbb{P}(n+2, n-1,3), \text { Diff }=\frac{1}{2} \Gamma+\frac{3}{4} \Gamma_{2}\end{array}$ & 6,12 \\
\hline 114 & $\begin{array}{c}z^{3} x^{2}+z x^{3} y+a z^{3} x y^{2}+ \\
b z^{5} y+c z x^{2} y^{3}+d z x y^{5}+ \\
+e z y^{7}\end{array}$ & $\begin{array}{c}t+g_{5}+g(z, x, y) \subset \mathbb{P}(17,3,4,2) \\
\mathbb{P}(3,2,1), \text { Diff }=\frac{1}{2} \Gamma+\frac{3}{4} \Gamma_{2}\end{array}$ & 4 \\
\hline
\end{tabular}

6. Singularity $-t^{3}+g(z, x, y)$.

\begin{tabular}{|c|c|c|c|}
\hline 1 & $z^{2} x y+x^{5}+x y^{5}$ & $t+z^{2} x y+x^{5}+x y^{5} \subset \mathbb{P}(25,8,5,4)$ & 12 \\
& & $\mathbb{P}(2,5,1)$, Diff $=\frac{2}{3} \Gamma+\frac{11}{12} \Gamma_{3}$ & \\
\hline 2 & $z^{2} x y+x^{4} y+a t y^{4}+$ & $t^{3}+z x y+x^{4} y+a t y^{4}+b y^{6} \subset \mathbb{P}(8,15,5,4)$ & 8 \\
& $+b y^{6}$ & Diff $=\left(0, \frac{1}{2}, 0,0\right)+\frac{4}{5} \Gamma_{4}$ & \\
\hline 3 & $z^{2} x y+x^{4} y+a x^{2} y^{4}+$ & $t+z x y+x^{4} y+a x^{2} y^{4}+b y^{7} \subset \mathbb{P}(14,9,3,2)$ & 12 \\
& $+b y^{7}$ & $\mathbb{P}(3,1,2)$, Diff $=\left(0, \frac{1}{2}, 0,0\right)+\frac{2}{3} \Gamma+\frac{8}{9} \Gamma_{4}$ & \\
\hline 4 & $z^{2} x y+x^{4} y+y^{8}$ & $t+z x y+x^{4} y+y^{8} \subset \mathbb{P}(32,21,7,4)$ & 24 \\
& & $\mathbb{P}(3,1,4)$, Diff $=\left(0, \frac{1}{2}, 0,0\right)+\frac{2}{3} \Gamma+\frac{20}{21} \Gamma_{4}$ & \\
\hline
\end{tabular}




\begin{tabular}{|c|c|c|c|}
\hline 5 & $z^{2} x y+x^{4} y+x y^{5}$ & $\begin{array}{c}t+z^{2} x y+x^{4} y+x y^{5} \subset \mathbb{P}(19,6,4,3) \\
\mathbb{P}(1,2,1), \text { Diff }=\frac{2}{3} \Gamma+\frac{8}{9} \Gamma_{3}+\frac{5}{6} \Gamma_{4}\end{array}$ & 9 \\
\hline 6 & $z^{2} x y+x^{4} y+x y^{6}$ & $\begin{array}{l}t+z x y+x^{4} y+x y^{6} \subset \mathbb{P}(23,15,5,3) \\
\mathbb{P}^{2}, \text { Diff }=\left(0, \frac{1}{2}, 0,0\right)+\frac{2}{3} \Gamma+\frac{8}{9} \Gamma_{3}+\frac{14}{15} \Gamma_{4}\end{array}$ & 18 \\
\hline 7 & $\begin{array}{c}z^{2} x y+x^{4} y+z y^{n} \\
n=4 ; 5\end{array}$ & $\begin{array}{c}t+g(z, x, y) \subset \mathbb{P}(8 n-3,3 n-3,2 n-2,5) \\
\mathbb{P}(3,2,5), \text { Diff }=\frac{2}{3} \Gamma+\frac{3 n-4}{3 n-3} \Gamma_{4}\end{array}$ & 9,15 \\
\hline 8 & $z^{2} x y+x^{4} y+t y^{5}$ & $\begin{array}{c}t^{3}+z x y+x^{4} y+t y^{5} \subset \mathbb{P}(20,39,13,8) \\
\text { Diff }=\left(0, \frac{1}{2}, 0,0\right)+\frac{12}{13} \Gamma_{4}\end{array}$ & 16 \\
\hline 9 & $z^{2} x y+x^{4} y+t x y^{3}$ & $\begin{array}{c}t^{3}+z x y+x^{4} y+t x y^{3} \subset \mathbb{P}(11,21,7,5) \\
\text { Diff }=\left(0, \frac{1}{2}, 0,0\right)+\frac{6}{7} \Gamma_{4}\end{array}$ & 10 \\
\hline 10 & $\begin{array}{c}z^{4}+z x^{2} y+z y^{n} \\
n=4 ; 8\end{array}$ & $\begin{array}{l}t+z^{4}+z x y+z y^{n} \subset \mathbb{P}(4 n, n, 3 n-3,3) \\
\mathbb{P}(n, n-1,1), \text { Diff }=\left(0,0, \frac{1}{2}, 0\right)+\frac{2}{3} \Gamma+\frac{8}{9} \Gamma_{2} \\
\end{array}$ & 9,18 \\
\hline 11 & $\begin{array}{c}z^{4}+z x^{2} y+z y^{2 n+1} \\
n=2,3\end{array}$ & $\begin{array}{c}t+g(z, x, y) \subset \mathbb{P}(8 n+4,2 n+1,3 n, 3) \\
\mathbb{P}(2 n+1, n, 1), \text { Diff }=\frac{2}{3} \Gamma+\frac{8}{9} \Gamma_{2}\end{array}$ & 9 \\
\hline 12 & $z^{4}+t x^{i} f_{3-i}(x, y)$ & $\begin{array}{c}t^{3}+z+t x^{i} f_{3-i}(x, y) \subset \mathbb{P}(3,9,2,2) \\
\mathbb{P}(3,1,1), \text { Diff }=\frac{3}{4} \Delta+\frac{7}{8} \Gamma_{2}\end{array}$ & 8 \\
\hline 13 & $\begin{array}{c}z^{3} x+z x^{2} y+a t z^{2} y+ \\
b t x y^{2}+c z y^{4},|a|+|c| \neq 0\end{array}$ & $\begin{array}{c}t^{3}+g(z, x, y) \subset \mathbb{P}(7,5,6,4) \\
\text { Diff }=\frac{1}{2} \Gamma_{2}\end{array}$ & 4 \\
\hline 14 & $\begin{array}{c}z^{3} x+z x^{2} y+a z x y^{3}+ \\
+b z y^{5}\end{array}$ & $\begin{array}{c}t+g(z, x, y) \subset \mathbb{P}(13,3,4,2) \\
\mathbb{P}(3,2,1), \text { Diff }=\frac{2}{3} \Gamma+\frac{5}{6} \Gamma_{2}\end{array}$ & 6 \\
\hline 15 & $z^{3} x+z x^{2} y+z y^{6}$ & $\begin{array}{c}t+z^{3} x+z x^{2} y+z y^{6} \subset \mathbb{P}(31,7,10,4) \\
\mathbb{P}(7,5,2), \text { Diff }=\frac{2}{3} \Gamma+\frac{5}{6} \Gamma_{2}\end{array}$ & 12 \\
\hline 16 & $\begin{array}{c}z^{3} x+z x^{2} y+t y^{n} \\
n=3 ; 5\end{array}$ & $\begin{array}{c}t^{3}+g(z, x, y) \subset \mathbb{P}(5 n, 3 n+2,6 n-6,10) \\
\text { Diff }=\frac{1}{2} \Gamma_{2}\end{array}$ & $\overline{4,10}$ \\
\hline 17 & $\begin{array}{c}z^{3} x+x^{3} y+a t z^{2} y+ \\
+b x y^{4}\end{array}$ & $\begin{array}{c}t^{3}+g(z, x, y) \subset \mathbb{P}(11,8,9,6) \\
\text { Diff }=\frac{1}{2} \Gamma_{3}\end{array}$ & 6 \\
\hline 18 & $z^{3} x+x^{3} y+z x y^{3}$ & $\begin{array}{c}t+z^{3} x+x^{3} y+z x y^{3} \subset \mathbb{P}(25,6,7,4) \\
\mathbb{P}(3,7,2), \text { Diff }=\frac{2}{3} \Gamma+\frac{5}{6} \Gamma_{3}\end{array}$ & 12 \\
\hline 19 & $z^{3} x+x^{3} y+t y^{3}$ & $\begin{array}{c}t^{3}+z x+x^{3} y+t y^{3} \subset \mathbb{P}(9,20,7,6) \\
\text { Diff }=\left(0, \frac{2}{3}, 0,0\right)+\frac{1}{2} \Gamma_{3}\end{array}$ & 4 \\
\hline 20 & $z^{3} x+x^{3} y+t z y^{2}$ & $\begin{array}{c}t^{3}+g(z, x, y) \subset \mathbb{P}(19,14,15,12) \\
\text { Diff }=\frac{1}{2} \Gamma_{3}\end{array}$ & 4 \\
\hline 21 & $z^{3} x+t x^{3}+x y^{4}$ & $\begin{array}{c}t^{3}+z x+t x^{3}+x y \subset \mathbb{P}(3,7,2,7) \\
\text { Diff }=\left(0, \frac{2}{3}, 0, \frac{3}{4}\right)+\frac{6}{7} \Gamma_{3}\end{array}$ & 21 \\
\hline 22 & $\begin{array}{c}z^{3} x+t x^{i} y^{j} f_{3-i-j}(x, y) \\
i=0 \& j \leq 1\end{array}$ & $\begin{array}{c}t^{3}+z x+t x^{i} y^{j} f_{3-i-j}(x, y) \subset \mathbb{P}(3,7,2,2) \\
\text { Diff }=\left(0, \frac{2}{3}, 0,0\right)+\frac{5}{6} \Gamma_{2}\end{array}$ & 6 \\
\hline 23 & $z^{3} x+t x^{2} y+x y^{4}$ & $\begin{array}{c}t^{3}+z x+t x^{2} y+x y^{4} \subset \mathbb{P}(7,16,5,4) \\
\text { Diff }=\left(0, \frac{2}{3}, 0,0\right)+\frac{3}{4} \Gamma_{3}\end{array}$ & 12 \\
\hline 24 & $z^{3} x+t x^{2} y+z y^{4}$ & $\begin{array}{c}t^{3}+g(z, x, y) \subset \mathbb{P}(25,19,18,14) \\
\text { Diff }=\frac{1}{2} \Gamma_{2}\end{array}$ & 14 \\
\hline 25 & $z^{3} x+t x^{2} y+t z y^{2}$ & $\begin{array}{c}t^{3}+g(z, x, y) \subset \mathbb{P}(13,10,9,8) \\
\text { Diff }=\frac{1}{2} \Gamma_{3}\end{array}$ & 8 \\
\hline 26 & $\begin{array}{c}z^{3} y+t x^{3}+a t z y^{2}+ \\
+b y^{5}\end{array}$ & $\begin{array}{c}t^{3}+z^{3} y+t x+a t z y^{2}+b y^{5} \subset \mathbb{P}(5,4,10,3) \\
\text { Diff }=\left(0,0, \frac{2}{3}, 0\right)+\frac{1}{2} \Gamma_{4}\end{array}$ & 9 \\
\hline
\end{tabular}




\begin{tabular}{|c|c|c|c|}
\hline 27 & $z^{3} y+t x^{3}+x^{2} y^{3}$ & $\begin{array}{c}t^{3}+z y+t x^{3}+x^{2} y^{3} \subset \mathbb{P}(9,22,6,5) \\
\text { Diff }=\left(0, \frac{2}{3}, 0,0\right)+\frac{1}{2} \Gamma_{4}\end{array}$ & 15 \\
\hline 28 & $z^{3} y+t x^{3}+z y^{4}$ & $\begin{array}{c}t^{3}+z^{3} y+t x+z y^{4} \subset \mathbb{P}(11,9,22,6) \\
\text { Diff }=\left(0,0, \frac{2}{3}, 0\right)+\frac{1}{2} \Gamma_{2}\end{array}$ & 18 \\
\hline 29 & $\begin{array}{l}t z^{i} f_{3-i}(z, y)+\frac{z^{2} x^{2}}{2}+ \\
\quad+z x^{2} y+a x^{2} y^{2} \\
(a \neq 0 \& i \leq 2) \| i=0\end{array}$ & $\begin{array}{c}t^{3}+g\left(z, x^{1 / 2}, y\right) \subset \mathbb{P}(3,2,5,2) \\
\text { Diff }=\frac{1}{2} \Upsilon+\frac{3}{4} \Gamma_{3}\end{array}$ & 4 \\
\hline 30 & $t z^{3}+z x^{3}+x y^{3}$ & $\begin{array}{c}t^{3}+t z^{3}+z x^{3}+x y \subset \mathbb{P}(9,6,7,20) \\
\text { Diff }=\left(0,0,0, \frac{2}{3}\right)+\frac{1}{2} \Gamma_{3}\end{array}$ & 4 \\
\hline 31 & $t z^{3}+z x^{3}+z y^{4}$ & $\begin{array}{c}t^{3}+t z^{3}+z x+z y \subset \mathbb{P}(3,2,7,7) \\
\text { Diff }=\left(0,0, \frac{2}{3}, \frac{3}{4}\right)+\frac{6}{7} \Gamma_{2}\end{array}$ & 21 \\
\hline 32 & $\begin{array}{c}t z^{3}+z x^{2} y+x y^{n} \\
n=3 ; 5\end{array}$ & $\begin{array}{c}t^{3}+g(z, x, y) \subset \mathbb{P}(6 n-3,4 n-2,7 n-9,11) \\
\text { Diff }=\frac{1}{2} \Gamma_{4}\end{array}$ & 4,11 \\
\hline 33 & $\begin{array}{l}t z^{3}+z x^{2} y+z y^{n} \\
n=4 ; 6\end{array}$ & $\begin{array}{c}t^{3}+t z^{3}+z x y+z y^{n} \subset \mathbb{P}(3 n, 2 n, 7 n-7,7) \\
\text { Diff }=\left(0,0, \frac{1}{2}, 0\right)+\frac{6}{7} \Gamma_{2}\end{array}$ & 7,14 \\
\hline 34 & $t z^{3}+z x^{2} y+z y^{5}$ & $\begin{array}{c}t^{3}+t z^{3}+z x^{2} y+z y^{5} \subset \mathbb{P}(15,10,14,7) \\
\text { Diff }=\frac{6}{7} \Gamma_{2}+\frac{1}{2} \Gamma_{4}\end{array}$ & 7 \\
\hline 35 & $\begin{array}{l}\frac{a t z^{3}+t x y^{2}+z x^{2} y+}{+b z^{2} y^{3},} a \neq 0 \\
\quad \neq 0\end{array}$ & $\begin{array}{c}t^{3}+g(z, x, y) \subset \mathbb{P}(9,6,8,5) \\
\text { Diff }=\frac{1}{2} \Gamma_{4}\end{array}$ & 5 \\
\hline 36 & $t z^{3}+z x^{2} y+t y^{5}$ & $\begin{array}{c}t^{3}+t z^{3}+z x y+t y^{5} \subset \mathbb{P}(15,10,29,6) \\
\text { Diff }=\frac{1}{2} \Upsilon+\frac{3}{4} \Gamma_{3}\end{array}$ & 12 \\
\hline 37 & $t z^{3}+z x^{2} y+t z y^{3}$ & $\begin{array}{c}t^{3}+t z^{3}+z x y+t z y^{3} \subset \mathbb{P}(9,6,17,4) \\
\text { Diff }=\frac{1}{2} \Upsilon+\frac{3}{4} \Gamma_{3}\end{array}$ & 8 \\
\hline 38 & $t z^{2} x+z x^{2} y+y^{5}$ & $\begin{array}{c}t^{3}+g(z, x, y) \subset \mathbb{P}(15,8,14,9) \\
\text { Diff }=\frac{1}{2} \Gamma_{4}\end{array}$ & 8 \\
\hline 39 & $t z^{2} x+z x^{2} y+z y^{4}$ & $\begin{array}{c}t^{3}+g(z, x, y) \subset \mathbb{P}(13,7,12,8) \\
\text { Diff }=\frac{3}{4} \Gamma_{2}\end{array}$ & 8 \\
\hline$\overline{40}$ & $t z^{2} x+x^{2} y^{2}+z^{3} y^{2}$ & $\begin{array}{c}t^{3}+t z^{2} x+x^{2} y+z^{3} y \subset \mathbb{P}(7,4,6,9) \\
\text { Diff }=\frac{1}{2} \Omega+\frac{3}{4} \Gamma_{4}\end{array}$ & 8 \\
\hline$\overline{41}$ & $\begin{array}{l}g_{3}(t, z)+z x^{3}+ \\
+\left(z y^{5} \| t y^{5}\right)\end{array}$ & $\begin{array}{c}g_{3}(t, z)+z x+(z y \| t y) \subset \mathbb{P}(1,1,2,2) \\
\text { Diff }=\left(0,0, \frac{2}{3}, \frac{4}{5}\right)+\frac{1}{2} \Gamma_{2}\end{array}$ & 6 \\
\hline 42 & $\begin{array}{l}g_{3}(t, z)+z x^{3}+ \\
+\left(z x y^{3} \| t x y^{3}\right)\end{array}$ & $\begin{array}{c}g_{3}(t, z)+z x^{3}+(z x y \| t x y) \subset \mathbb{P}(3,3,2,4) \\
\text { Diff }=\left(0,0,0, \frac{2}{3}\right)+\frac{1}{2} \Gamma_{2}\end{array}$ & 4 \\
\hline 43 & $\begin{array}{l}z^{2} x+x^{4}+x y^{2 n} \\
n=4,5,7,8\end{array}$ & $\begin{array}{c}t+z^{2} x+x^{4}+x y^{2} \subset \mathbb{P}(8,3,2,3) \\
\mathbb{P}(1,2,1), \text { Diff }=\left(0,0,0, \frac{n-1}{n}\right)+\frac{2}{3} \Gamma+\frac{8}{9} \Gamma_{3}\end{array}$ & 9 \\
\hline 44 & $\begin{array}{l}z^{2} x+x^{4}+x y^{n} \\
n=7 ; 11 ; 13,17\end{array}$ & $\begin{array}{c}t+z x+x^{4}+x y \subset \mathbb{P}(4,3,1,3) \\
\mathbb{P}^{2}, \text { Diff }=\left(0, \frac{1}{2}, 0, \frac{n-1}{n}\right)+\frac{2}{3} \Gamma+\frac{8}{9} \Gamma_{3}\end{array}$ & $9,12,18$ \\
\hline 45 & $\begin{array}{c}z^{2} x+x^{5}+x y^{n} \\
n=7 ; 11\end{array}$ & $\begin{array}{c}t+z^{2} x+x^{5}+x y \subset \mathbb{P}(5,2,1,4) \\
\mathbb{P}(1,1,2), \text { Diff }=\left(0,0,0, \frac{n-1}{n}\right)+\frac{2}{3} \Gamma+\frac{5}{6} \Gamma_{3}\end{array}$ & 7,12 \\
\hline 46 & $\begin{array}{l}z^{2} x+t y^{2}\left(a z+b x^{2}+\right. \\
\left.+c y^{3}\right)+x^{5}+d x^{3} y^{3}+ \\
+e x y^{6},|a|+|c| \neq 0\end{array}$ & $\begin{array}{c}t^{3}+g(z, x, y) \subset \mathbb{P}(5,6,3,2) \\
\text { Diff }=\frac{1}{2} \Gamma_{3}\end{array}$ & 2 \\
\hline 47 & $\begin{aligned} z^{2} x+ & t y^{3}\left(a z+b x^{2}\right)+ \\
& +x^{5}+c x y^{9}\end{aligned}$ & $\begin{array}{c}t^{3}+g\left(z, x, y^{1 / 3}\right) \subset \mathbb{P}(5,6,3,4) \\
\text { Diff }=\left(0,0,0, \frac{2}{3}\right)+\frac{1}{2} \Gamma_{3}\end{array}$ & 4 \\
\hline
\end{tabular}




\begin{tabular}{|c|c|c|c|}
\hline 48 & $\begin{aligned} z^{2} x+ & x^{5}+a x^{3} y^{5}+ \\
& +x y^{10}\end{aligned}$ & $\begin{array}{c}t+z^{2} x+x^{5}+a x^{3} y+x y^{2} \subset \mathbb{P}(5,2,1,2) \\
\mathbb{P}^{2}, \text { Diff }=\left(0,0,0, \frac{4}{5}\right)+\frac{2}{3} \Gamma+\frac{5}{6} \Gamma_{3}\end{array}$ & 6 \\
\hline 49 & $\begin{array}{c}z^{2} x+x^{5}+t y^{n} \\
n=7 ; 9\end{array}$ & $\begin{array}{c}t^{3}+z^{2} x+x^{5}+t y \subset \mathbb{P}(5,6,3,10) \\
\text { Diff }=\left(0,0,0, \frac{n-1}{n}\right)+\frac{1}{2} \Gamma_{3}\end{array}$ & 7,10 \\
\hline 50 & $\begin{array}{l}z^{2} x+x^{4} y+x y^{n} \\
\quad n=9 ; 11 ; 15\end{array}$ & $\begin{array}{l}t+g\left(z^{1 / 2}, x, y\right) \subset \mathbb{P}(4 n-1,3 n, n-1,3) \\
\mathbb{P}(n, n-1,1), \text { Diff }=\left(0, \frac{1}{2}, 0,0\right)+\frac{2}{3} \Gamma+\frac{8}{9} \Gamma_{3}\end{array}$ & $10,12,18$ \\
\hline 51 & $\begin{array}{c}z^{2} x+x^{4} y+x y^{2 n} \\
n=4,6,7\end{array}$ & $\begin{array}{c}t+g(z, x, y) \subset \mathbb{P}(8 n-1,3 n, 2 n-1,3) \\
\mathbb{P}(n, 2 n-1,1), \text { Diff }=\frac{2}{3} \Gamma+\frac{8}{9} \Gamma_{3}\end{array}$ & 9 \\
\hline 52 & 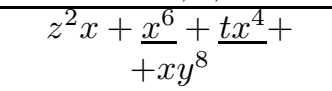 & $\begin{array}{c}t^{3}+z^{2} x+\underline{x^{6}}+\underline{t x^{4}}+x y^{2} \subset \mathbb{P}(4,5,2,5) \\
\text { Diff }=\left(0,0,0, \frac{3}{4}\right)+\frac{4}{5} \Gamma_{3}\end{array}$ & 5 \\
\hline 53 & $\begin{array}{l}z^{2} x+\underline{x^{6}}+\underline{t x^{4}}+ \\
+x y^{n}, n=7 ; 9\end{array}$ & $\begin{array}{c}t^{3}+z x+\underline{x^{6}}+\frac{t x^{4}}{+x y} \subset \mathbb{P}(2,5,1,5) \\
\text { Diff }=\left(0, \frac{1}{2}, 0, \frac{n-1}{n}\right)+\frac{4}{5} \Gamma_{3}\end{array}$ & 8,10 \\
\hline 54 & $z^{2} x+x^{5} y+x y^{10}$ & $\begin{array}{c}t+z^{2} x+x^{5} y+x y^{10} \subset \mathbb{P}(49,20,9,4) \\
\mathbb{P}(5,9,1), \text { Diff }=\frac{2}{3} \Gamma+\frac{11}{12} \Gamma_{3}\end{array}$ & 12 \\
\hline 55 & $\begin{array}{c}z^{2} x+x^{5} y+a t x^{2} y^{3}+ \\
+x y^{8}\end{array}$ & $\begin{array}{c}t^{3}+g(z, x, y) \subset \mathbb{P}(13,16,7,4) \\
\text { Diff }=\frac{3}{4} \Gamma_{3}\end{array}$ & 4 \\
\hline 56 & $\begin{array}{c}z^{2} x+x^{5} y+t z y^{n} \\
n=2,3\end{array}$ & $\begin{array}{c}t^{3}+g(z, x, y) \subset \mathbb{P}(10 n+1,12 n+2,6 n-1,8) \\
\text { Diff }=\frac{1}{2} \Gamma_{3}\end{array}$ & 8 \\
\hline 57 & $z^{2} x+x^{4} y^{2}+x y^{10}$ & $\begin{array}{c}t+z^{2} x+x^{4} y^{2}+x y^{10} \subset \mathbb{P}(38,15,8,3) \\
\mathbb{P}(5,8,1), \text { Diff }=\frac{2}{3} \Gamma+\frac{8}{9} \Gamma_{3}\end{array}$ & 9 \\
\hline 58 & $z^{2} x+x^{4} y^{2}+x y^{12}$ & $\begin{array}{c}t+z^{2} x+x^{4} y+x y^{6} \subset \mathbb{P}(23,9,5,3) \\
\mathbb{P}(3,5,1), \text { Diff }=\left(0,0,0, \frac{1}{2}\right)+\frac{2}{3} \Gamma+\frac{8}{9} \Gamma_{3}\end{array}$ & 9 \\
\hline 59 & $\begin{array}{l}z^{2} x+x^{4} y^{2}+x y^{n} \\
\quad n=7 ; 9 ; 13\end{array}$ & $\begin{array}{c}t+z x+x^{4} y^{2}+x y^{n} \subset \mathbb{P}(4 n-2,3 n, n-2,3) \\
\quad \mathbb{P}(n, n-2,1), \text { Diff }=\left(0, \frac{1}{2}, 0,0\right)+\frac{2}{3} \Gamma+\frac{8}{9} \Gamma_{3}\end{array}$ & $9,12,18$ \\
\hline 60 & $z^{2} x+x^{7}+x y^{7}$ & $\begin{array}{c}t+z^{2} x+x^{7}+x y \subset \mathbb{P}(7,3,1,6) \\
\mathbb{P}(1,1,2), \text { Diff }=\left(0,0,0, \frac{6}{7}\right)+\frac{2}{3} \Gamma+\frac{8}{9} \Gamma_{3}\end{array}$ & 9 \\
\hline 61 & $\begin{array}{c}z^{2} x+\frac{x^{7}}{+x y^{8}}+x^{4} y^{4}+ \\
\end{array}$ & 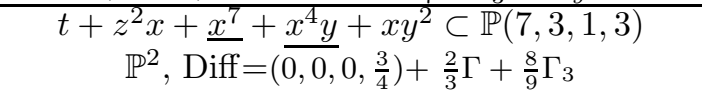 & 9 \\
\hline 62 & $\begin{array}{c}z^{2} x+x^{6} y+x y^{n} \\
n=7 ; 9\end{array}$ & $\begin{array}{c}t+z x+x^{6} y+x y^{n} \subset \mathbb{P}(6 n-1,5 n, n-1,5) \\
\mathbb{P}(n, n-1,1), \text { Diff }=\left(0, \frac{1}{2}, 0,0\right)+\frac{2}{3} \Gamma+\frac{14}{15} \Gamma_{3}\end{array}$ & 15,30 \\
\hline 63 & $z^{2} x+x^{6} y+x y^{8}$ & $\begin{array}{c}t+z^{2} x+x^{6} y+x y^{8} \subset \mathbb{P}(47,20,7,5) \\
\mathbb{P}(4,7,1), \text { Diff }=\frac{2}{3} \Gamma+\frac{14}{15} \Gamma_{3}\end{array}$ & 15 \\
\hline 64 & $\begin{array}{l}z^{2} x+x^{5} y^{2}+t y^{2}(a z \\
\left.\quad+b x^{2} y\right)+c x y^{7}\end{array}$ & $\begin{array}{c}t^{3}+g(z, x, y) \subset \mathbb{P}(11,14,5,4) \\
\text { Diff }=\frac{1}{2} \Gamma_{3}\end{array}$ & 4 \\
\hline 65 & $\begin{array}{l}z^{2} x+x^{5} y^{2}+a x^{3} y^{5}+ \\
+x y^{8}\end{array}$ & $\begin{array}{c}t+g(z, x, y) \subset \mathbb{P}(19,8,3,2) \\
\mathbb{P}(4,3,1), \text { Diff }=\frac{2}{3} \Gamma+\frac{5}{6} \Gamma_{3}\end{array}$ & 6 \\
\hline 66 & $z^{2} x+x^{5} y^{2}+x y^{9}$ & $\begin{array}{c}t+z^{2} x+x^{5} y^{2}+x y^{9} \subset \mathbb{P}(43,18,7,4) \\
\mathbb{P}(9,7,2), \text { Diff }=\frac{2}{3} \Gamma+\frac{5}{6} \Gamma_{3}\end{array}$ & 12 \\
\hline 67 & $\begin{array}{c}z^{2} x+x^{5} y^{2}+t y^{n} \\
n=5 ; 7\end{array}$ & $\begin{array}{c}t^{3}+g(z, x, y) \subset \mathbb{P}(5 n, 6 n+2,3 n-4,10) \\
\text { Diff }=\frac{1}{2} \Gamma_{3}\end{array}$ & 7,10 \\
\hline 68 & $\begin{array}{c}z^{2} x+x^{4} y^{3}+x y^{n} \\
n=7 ; 11\end{array}$ & $\begin{array}{c}t+z x+x^{4} y^{3}+x y^{n} \subset \mathbb{P}(4 n-3,3 n, n-3,3) \\
\mathbb{P}(n, n-3,1), \text { Diff }=\left(0, \frac{1}{2}, 0,0\right)+\frac{2}{3} \Gamma+\frac{8}{9} \Gamma_{3}\end{array}$ & 10,18 \\
\hline 69 & $\begin{array}{c}z^{2} x+x^{4} y^{3}+x y^{2 n} \\
n=4,5\end{array}$ & $\begin{array}{c}t+g(z, x, y) \subset \mathbb{P}(8 n-3,3 n, 2 n-3,3) \\
\mathbb{P}(n, 2 n-3,1), \text { Diff }=\frac{2}{3} \Gamma+\frac{8}{9} \Gamma_{3}\end{array}$ & 9 \\
\hline
\end{tabular}




\begin{tabular}{|c|c|c|c|}
\hline 70 & $z^{2} x+x^{8}+x y^{8}$ & $\begin{array}{c}t+z^{2} x+x^{8}+x y^{2} \subset \mathbb{P}(16,7,2,7) \\
\mathbb{P}(1,2,1), \text { Diff }=\left(0,0,0, \frac{3}{4}\right)+\frac{2}{3} \Gamma+\frac{20}{21} \Gamma_{3} \\
\end{array}$ & 21 \\
\hline 71 & $z^{2} x+x^{7} y+x y^{8}$ & $\begin{array}{c}t+z^{2} x+x^{7} y+x y^{8} \subset \mathbb{P}(55,24,7,6) \\
\mathbb{P}(4,7,1), \text { Diff }=\frac{2}{3} \Gamma+\frac{17}{18} \Gamma_{3}\end{array}$ & 18 \\
\hline 72 & $z^{2} x+x^{7} y+t z y^{2}$ & $\begin{array}{c}t^{3}+z^{2} x+x^{7} y+t z y^{2} \subset \mathbb{P}(29,38,11,10) \\
\text { Diff }=\frac{1}{2} \Gamma_{3}\end{array}$ & 10 \\
\hline 73 & $z^{2} x+x^{6} y^{2}+x y^{8}$ & $\begin{array}{c}t+z^{2} x+x^{6} y+x y^{4} \subset \mathbb{P}(23,10,3,5) \\
\mathbb{P}(2,3,1), \text { Diff }=\left(0,0,0, \frac{1}{2}\right)+\frac{2}{2} \Gamma+\frac{14}{15} \Gamma_{3}\end{array}$ & 15 \\
\hline 74 & $z^{2} x+x^{5} y^{3}+x y^{8}$ & $\begin{array}{c}t+z^{2} x+x^{5} y^{3}+x y^{8} \subset \mathbb{P}(37,16,5,4) \\
\mathbb{P}(4,5,1), \text { Diff }=\frac{2}{2} \Gamma+\frac{11}{12} \Gamma_{3}\end{array}$ & 12 \\
\hline 75 & $z^{2} x+x^{5} y^{3}+t z y^{2}$ & $\begin{array}{c}t^{3}+z^{2} x+x^{5} y^{3}+t z y^{2} \subset \mathbb{P}(23,30,9,8) \\
\text { Diff }=\frac{1}{2} \Gamma_{3}\end{array}$ & 8 \\
\hline 76 & $z^{2} x+x^{4} y^{4}+x y^{9}$ & $\begin{array}{l}t+z x+x^{4} y^{4}+x y^{9} \subset \mathbb{P}(32,27,5,3) \\
\mathbb{P}(9,5,1), \text { Diff }=\left(0, \frac{1}{2}, 0,0\right)+\frac{2}{3} \Gamma+\frac{8}{9} \Gamma_{3}\end{array}$ & 18 \\
\hline 77 & $\begin{array}{c}z^{2} x+t x^{3}+x y^{2 n} \\
n=4,5,6\end{array}$ & $\begin{array}{c}t^{3}+z^{2} x+t x^{3}+x y^{2} \subset \mathbb{P}(6,7,4,7) \\
\text { Diff }=\left(0,0,0, \frac{n-1}{n}\right)+\frac{6}{7} \Gamma_{3}\end{array}$ & 7 \\
\hline 78 & $\begin{array}{c}z^{2} x+t x^{3}+x y^{n} \\
n=9 ; 11,13\end{array}$ & $\begin{array}{c}t^{3}+z x+t x^{3}+x y \subset \mathbb{P}(3,7,2,7) \\
\text { Diff }=\left(0, \frac{1}{2}, 0, \frac{n-1}{n}\right)+\frac{6}{7} \Gamma_{3}\end{array}$ & 10,14 \\
\hline 79 & $z^{2} x+t x^{i} f_{3-i}\left(x, y^{3}\right)$ & $\begin{array}{c}t^{3}+z x+t x^{i} f_{3-i}(x, y) \subset \mathbb{P}(3,7,2,2) \\
\text { Diff }=\left(0,0,0, \frac{2}{3}\right)+\frac{1}{2} \Delta+\frac{3}{4} \Gamma_{2}\end{array}$ & 4 \\
\hline 80 & $\begin{array}{l}z^{2} x+t x^{3}+t y^{n} \\
n=5 ; 7 ; 11\end{array}$ & $\begin{array}{c}t^{3}+z x+t x^{3}+t y \subset \mathbb{P}(3,7,2,6) \\
\text { Diff }=\left(0,0,0, \frac{n-1}{n}\right)+\frac{1}{2} \Delta+\frac{3}{4} \Gamma_{2}\end{array}$ & $5,8,12$ \\
\hline 81 & $\begin{array}{c}z^{2} x+t x^{3}+t x y^{n} \\
n=5,7\end{array}$ & $\begin{array}{c}t^{3}+z x+t x^{3}+t x y \subset \mathbb{P}(3,7,2,4) \\
\text { Diff }=\left(0,0,0, \frac{n-1}{n}\right)+\frac{1}{2} \Delta+\frac{3}{4} \Gamma_{2}\end{array}$ & 8 \\
\hline 82 & $\begin{array}{c}z^{2} x+t x^{3} y+x y^{2 n} \\
n=4,5\end{array}$ & $\begin{aligned} & t^{3}+g(z, x, y) \subset \mathbb{P}(6 n-1,7 n, 4 n-3,7) \\
& \operatorname{Diff}=\frac{6}{7} \Gamma_{3}\end{aligned}$ & 7 \\
\hline 83 & $\begin{array}{c}z^{2} x+t x^{3} y+x y^{n} \\
n=7 ; 9 ; 11\end{array}$ & $\begin{array}{c}t^{3}+g\left(z^{1 / 2}, x, y\right) \subset \mathbb{P}(3 n-1,7 n, 2 n-3,7) \\
\text { Diff }=\left(0, \frac{1}{2}, 0,0\right)+\frac{6}{7} \Gamma_{3}\end{array}$ & $8,10,14$ \\
\hline 84 & $z^{2} x+t y f_{3}\left(x, y^{2}\right)$ & $\begin{array}{c}t^{3}+z x+t y f_{3}\left(x, y^{2}\right) \subset \mathbb{P}(7,17,4,2) \\
\text { Diff }=\frac{1}{2} \Delta+\frac{3}{4} \Gamma_{2}\end{array}$ & 4 \\
\hline 85 & $\begin{array}{c}z^{2} x+t x^{3} y+t y^{n} \\
n=5 ; 9\end{array}$ & $\begin{aligned} t^{3}+g\left(z^{1 / 2}, x, y\right) & \subset \mathbb{P}(3 n, 7 n+2,2 n-2,6) \\
\text { Diff } & =\frac{1}{2} \Delta+\frac{3}{4} \Gamma_{2}\end{aligned}$ & 6,12 \\
\hline 86 & $z^{2} x+t x^{3} y+t x y^{6}$ & $\begin{array}{c}t^{3}+z x+t x^{3} y+t x y^{6} \subset \mathbb{P}(17,41,10,4) \\
\text { Diff }=\frac{1}{2} \Delta+\frac{3}{4} \Gamma_{2}\end{array}$ & 8 \\
\hline 87 & $z^{2} x+t x^{5}+x y^{7}$ & $\begin{array}{c}t^{3}+z x+t x^{5}+x y \subset \mathbb{P}(5,13,2,13) \\
\text { Diff }=\left(0, \frac{1}{2}, 0, \frac{6}{7}\right)+\frac{12}{13} \Gamma_{3}\end{array}$ & 14 \\
\hline 88 & $z^{2} x+t x^{5}+x y^{8}$ & $\begin{array}{c}t^{3}+z^{2} x+t x^{5}+x y^{2} \subset \mathbb{P}(10,13,4,13) \\
\text { Diff }=\left(0,0,0, \frac{3}{4}\right)+\frac{12}{13} \Gamma_{3}\end{array}$ & 13 \\
\hline 89 & $\begin{array}{c}z^{2} x+t x^{i} y^{j} f_{5-i-j}(x, y) \\
i=0 \& j \leq 2\end{array}$ & $\begin{array}{c}t^{3}+z x+t x^{i} y^{j} f_{5-i-j}(x, y) \subset \mathbb{P}(5,13,2,2) \\
\text { Diff }=\frac{1}{2} \Delta+\frac{3}{4} \Gamma_{2}\end{array}$ & 4 \\
\hline 90 & $z^{2} x+t x^{5}+t x y^{5}$ & $\begin{array}{c}t^{3}+z x+t x^{5}+t x y \subset \mathbb{P}(5,13,2,8) \\
\text { Diff }=\left(0,0,0, \frac{4}{5}\right)+\frac{1}{2} \Delta+\frac{3}{4} \Gamma_{2}\end{array}$ & 16 \\
\hline 91 & $z^{2} x+t x^{4} y+x y^{7}$ & $\begin{array}{c}t^{3}+z^{2} x+t x^{4} y+x y^{7} \subset \mathbb{P}(27,35,11,10) \\
\text { Diff }=\frac{4}{5} \Gamma_{3}\end{array}$ & 5 \\
\hline
\end{tabular}




\begin{tabular}{|c|c|c|c|}
\hline 92 & $z^{2} x+t x^{4} y+x y^{8}$ & $\begin{array}{c}t^{3}+z^{2} x+t x^{4} y+x y^{8} \subset \mathbb{P}(31,40,13,10) \\
\text { Diff }=\frac{9}{10} \Gamma_{3}\end{array}$ & 10 \\
\hline 93 & $\begin{aligned} z^{2} x+ & t x^{4} y+t z y^{2}+ \\
& +b x^{3} y^{5}\end{aligned}$ & $\begin{array}{c}t^{3}+g(z, x, y) \subset \mathbb{P}(17,22,7,6) \\
\text { Diff }=\frac{1}{2} \Gamma_{3}\end{array}$ & 6 \\
\hline 94 & $z^{2} x+t x^{4} y+t x y^{5}$ & $\begin{array}{c}t^{3}+z x+t x^{4} y+t x y^{5} \subset \mathbb{P}(19,49,8,6) \\
\text { Diff }=\frac{1}{2} \Delta+\frac{3}{4} \Gamma_{2}\end{array}$ & 12 \\
\hline 95 & $\begin{array}{c}z^{2} x+t x^{3} y^{2}+x y^{n} \\
n=7 ; 9\end{array}$ & $\begin{array}{c}t^{3}+g\left(z^{1 / 2}, x, y\right) \subset \mathbb{P}(3 n-2,7 n, 2 n-6,7) \\
\text { Diff }=\left(0, \frac{1}{2}, 0,0\right)+\frac{6}{7} \Gamma_{3}\end{array}$ & 10,14 \\
\hline 96 & $z^{2} x+t x^{3} y^{2}+x y^{8}$ & $\begin{array}{c}t^{3}+z^{2} x+t x^{3} y+x y^{4} \subset \mathbb{P}(11,14,5,7) \\
\text { Diff }=\left(0,0,0, \frac{1}{2}\right)+\frac{6}{7} \Gamma_{3}\end{array}$ & 7 \\
\hline 97 & $z^{2} x+t x^{3} y^{2}+t y^{7}$ & $\begin{array}{c}t^{3}+z x+t x^{3} y^{2}+t y^{7} \subset \mathbb{P}(21,53,10,6) \\
\text { Diff }=\frac{1}{2} \Delta+\frac{3}{4} \Gamma_{2}\end{array}$ & 12 \\
\hline 98 & $z^{2} x+t x^{3} y^{2}+t x y^{5}$ & $\begin{array}{c}t^{3}+z x+t x^{3} y^{2}+t x y^{5} \subset \mathbb{P}(13,33,6,4) \\
\text { Diff }=\frac{1}{2} \Delta+\frac{3}{4} \Gamma_{2}\end{array}$ & 8 \\
\hline 99 & $\begin{array}{c}z^{2} y+x^{7} y+t y^{2}(a z+ \\
\left.+b y^{4}\right)+c y^{9}\end{array}$ & $\begin{array}{c}t^{3}+g\left(z, x^{1 / 7}, y\right) \subset \mathbb{P}(3,4,8,1) \\
\text { Diff }=\left(0,0, \frac{6}{7}, 0\right)+\frac{3}{4} \Gamma_{4}\end{array}$ & 7 \\
\hline 100 & $\begin{array}{c}z^{2} y+x^{7} y+y^{n} \\
n=10 ; 14\end{array}$ & $\begin{array}{c}t+z y+x y+y^{n} \subset \mathbb{P}(n, n-1, n-1,1) \\
\mathbb{P}^{2}, \text { Diff }=\left(0, \frac{1}{2}, \frac{6}{7}, 0\right)+\frac{2}{3} \Gamma+\frac{3 n-4}{3 n-3} \Gamma_{4}\end{array}$ & 28,42 \\
\hline 101 & $\begin{array}{c}z^{2} y+x^{7} y+y^{2 n+1} \\
n=5 ; 6\end{array}$ & $\begin{array}{c}t+z^{2} y+x y+y^{2 n+1} \subset \mathbb{P}(2 n+1, n, 2 n, 1) \\
\mathbb{P}(1,2,1), \text { Diff }=\left(0,0, \frac{6}{7}, 0\right)+\frac{2}{3} \Gamma+\frac{3 n-1}{3 n} \Gamma_{4}\end{array}$ & 15,21 \\
\hline 102 & $\begin{aligned} z^{2} y+ & x^{7} y+a t y^{8}+ \\
& +b y^{12}\end{aligned}$ & $\begin{array}{c}t^{3}+z y+x y+a t y^{8}+b y^{12} \subset \mathbb{P}(4,11,11,1) \\
\text { Diff }=\left(0, \frac{1}{2}, \frac{6}{7}, 0\right)+\frac{10}{11} \Gamma_{4}\end{array}$ & 14 \\
\hline 103 & $\begin{array}{c}z^{2} y+x^{7} y+x y^{n} \\
n=8 ; 12\end{array}$ & $\begin{array}{c}t+g\left(z^{1 / 2}, x, y\right) \subset \mathbb{P}(7 n-1,7 n-7, n-1,6) \\
\mathbb{P}(7,1,6), \text { Diff }=\left(0, \frac{1}{2}, 0,0\right)+\frac{2}{3} \Gamma+\frac{3 n-4}{3 n-3} \Gamma_{4}\end{array}$ & 22,36 \\
\hline 104 & $\begin{array}{c}z^{2} y+x^{7} y+a x^{4} y^{5}+ \\
+b x y^{9}\end{array}$ & $\begin{array}{c}t+g(z, x, y) \subset \mathbb{P}(31,14,4,3) \\
\mathbb{P}(7,2,3), \text { Diff }=\frac{2}{3} \Gamma+\frac{5}{6} \Gamma_{4}\end{array}$ & 22 \\
\hline 105 & $z^{2} y+x y f_{3}\left(x^{2}, y^{3}\right)$ & $\begin{array}{c}t+z y+x y f_{3}\left(x^{2}, y^{3}\right) \subset \mathbb{P}(23,21,3,2) \\
\mathbb{P}(7,1,2), \text { Diff }=\left(0, \frac{1}{2}, 0,0\right)+\frac{2}{3} \Gamma+\frac{8}{9} \Gamma_{4}\end{array}$ & 12 \\
\hline 106 & $\begin{aligned} z^{2} y+ & x^{7} y+a x^{4} y^{6}+ \\
& +b x y^{11}\end{aligned}$ & $\begin{array}{c}t+z y+x^{7} y+a x^{4} y^{6}+b x y^{11} \subset \mathbb{P}(38,35,5,3) \\
\mathbb{P}(7,1,3), \text { Diff }=\left(0, \frac{1}{2}, 0,0\right)+\frac{2}{3} \Gamma+\frac{14}{15} \Gamma_{4}\end{array}$ & 18 \\
\hline 107 & $\begin{array}{c}z^{2} y+x^{7} y+x^{2} y^{2 n+1} \\
n=3 ; 4\end{array}$ & $\begin{array}{c}t+g(z, x, y) \subset \mathbb{P}(14 n+5,7 n, 2 n, 5) \\
\mathbb{P}(7,2,5), \text { Diff }=\frac{2}{3} \Gamma+\frac{3 n-1}{3 n} \Gamma_{4}\end{array}$ & 9,15 \\
\hline 108 & $\begin{array}{c}z^{2} y+x^{7} y+a t x^{3} y^{3}+ \\
+b x^{2} y^{8}\end{array}$ & $\begin{array}{c}t^{3}+g\left(z^{1 / 2}, x, y\right) \subset \mathbb{P}(18,49,7,5) \\
\text { Diff }=\left(0, \frac{1}{2}, 0,0\right)+\frac{6}{7} \Gamma_{4}\end{array}$ & 10 \\
\hline 109 & $z^{2} y+x^{7} y+x^{2} y^{10}$ & $\begin{array}{l}t+z y+x^{7} y+x^{2} y^{10} \subset \mathbb{P}(68,63,9,5) \\
\mathbb{P}(7,1,5), \text { Diff }=\left(0, \frac{1}{2}, 0,0\right)+\frac{2}{3} \Gamma+\frac{26}{27} \Gamma_{4}\end{array}$ & 30 \\
\hline 110 & $\begin{array}{c}z^{2} y+x^{7} y+a t x^{2} y^{4}+ \\
+b x^{3} y^{6}\end{array}$ & $\begin{array}{c}t^{3}+g\left(z^{1 / 2}, x, y\right) \subset \mathbb{P}(13,35,5,4) \\
\text { Diff }=\left(0, \frac{1}{2}, 0,0\right)+\frac{4}{5} \Gamma_{4}\end{array}$ & 8 \\
\hline 111 & $z^{2} y+x^{7} y+x^{3} y^{8}$ & $\begin{array}{l}t+z y+x^{7} y+x^{3} y^{8} \subset \mathbb{P}(53,49,7,4) \\
\mathbb{P}(7,1,4), \text { Diff }=\left(0, \frac{1}{2}, 0,0\right)+\frac{2}{3} \Gamma+\frac{20}{21} \Gamma_{4}\end{array}$ & 24 \\
\hline 112 & $\begin{array}{c}z^{2} y+x^{7} y+t y^{n} \\
n=7 ; 9\end{array}$ & $\begin{array}{c}t^{3}+z y+x y+t y^{n} \subset \mathbb{P}(n, 3 n-2,3 n-2,2) \\
\text { Diff }=\left(0, \frac{1}{2}, \frac{6}{7}, 0\right)+\frac{3 n-3}{3 n-2} \Gamma_{4}\end{array}$ & 22,28 \\
\hline 113 & $\begin{array}{c}z^{2} y+x^{7} y+t x y^{n} \\
n=5 ; 7\end{array}$ & $\begin{array}{c}t^{3}+g\left(z^{1 / 2}, x, y\right) \subset \mathbb{P}(7 n-1,21 n-14,3 n-2,11) \\
\text { Diff }=\left(0, \frac{1}{2}, 0,0\right)+\frac{3 n-3}{3 n-2} \Gamma_{4}\end{array}$ & $\overline{16,22}$ \\
\hline
\end{tabular}




\begin{tabular}{|c|c|c|c|}
\hline$\overline{114}$ & $z^{2} y+x^{7} y+t x y^{6}$ & $\begin{array}{c}t^{3}+z^{2} y+x^{7} y+t x y^{6} \subset \mathbb{P}(41,56,16,11) \\
\text { Diff }=\frac{7}{8} \Gamma_{4}\end{array}$ & 11 \\
\hline 115 & $z^{2} y+x^{7} y+t x^{2} y^{5}$ & $\begin{array}{c}t^{3}+z y+x^{7} y+t x^{2} y^{5} \subset \mathbb{P}(33,91,13,8) \\
\text { Diff }=\left(0, \frac{1}{2}, 0,0\right)+\frac{12}{12} \Gamma_{4}\end{array}$ & 16 \\
\hline 116 & $\begin{array}{l}z^{2} y+t x^{5}+t y^{2}(a z+ \\
\left.+b y^{4}\right)+c x^{5} y^{3}+d y^{9} \\
|a|+|b|+|d| \neq 0\end{array}$ & $\begin{array}{c}t^{3}+g\left(z, x^{1 / 5}, y\right) \subset \mathbb{P}(3,4,6,1) \\
\text { Diff }=\left(0,0, \frac{4}{5}, 0\right)+\frac{1}{2} \Gamma_{4}\end{array}$ & 5 \\
\hline 117 & $z^{2} y+t x^{5}+y^{13}$ & $\begin{array}{c}t^{3}+z^{2} y+t x+y^{13} \subset \mathbb{P}(13,18,26,3) \\
\text { Diff }=\left(0,0, \frac{4}{5}, 0\right)+\frac{1}{2} \Gamma_{4}\end{array}$ & 15 \\
\hline 118 & $\begin{array}{c}z^{2} y+t x^{5}+x y^{4 n+3} \\
n=1 ; 2\end{array}$ & $\begin{array}{c}t^{3}+g(z, x, y) \subset \mathbb{P}(20 n+15,30 n+16,8 n+6,13) \\
\text { Diff }=\frac{1}{2} \Gamma_{4}\end{array}$ & 9,13 \\
\hline 119 & $z^{2} y+t x^{5}+x^{2} y^{9}$ & $\begin{array}{c}t^{3}+z^{2} y+t x^{5}+x^{2} y^{9} \subset \mathbb{P}(45,62,18,11) \\
\text { Diff }=\frac{1}{2} \Gamma_{4}\end{array}$ & 11 \\
\hline 120 & $z^{2} y+t x^{5}+x^{3} y^{7}$ & $\begin{array}{c}t^{3}+z^{2} y+t x^{5}+x^{3} y^{7} \subset \mathbb{P}(35,48,14,9) \\
\text { Diff }=\frac{1}{2} \Gamma_{4}\end{array}$ & 9 \\
\hline 121 & $z^{2} y+t x^{5}+x^{4} y^{5}$ & $\begin{array}{c}t^{3}+z^{2} y+t x^{5}+x^{4} y^{5} \subset \mathbb{P}(25,34,10,7) \\
\text { Diff }=\frac{1}{2} \Gamma_{4}\end{array}$ & 7 \\
\hline$\overline{122}$ & $\begin{array}{c}z^{2} y+t x^{5}+t y^{n} \\
n=7 ; 9\end{array}$ & $\begin{array}{c}t^{3}+z y+t x+t y^{n} \subset \mathbb{P}(n, 3 n-2,2 n, 2) \\
\text { Diff }=\left(0,0, \frac{4}{5}, 0\right)+\frac{1}{2} \Delta+\frac{3}{4} \Gamma_{2}\end{array}$ & 16,20 \\
\hline 123 & $\begin{array}{c}z^{2} y+t x^{5}+t x y^{n} \\
n=5 ; 7\end{array}$ & $\begin{array}{c}t^{3}+z y+t x^{5}+t x y^{n} \subset \mathbb{P}(5 n, 15 n-8,2 n, 8) \\
\text { Diff }=\frac{1}{2} \Delta+\frac{3}{4} \Gamma_{2}\end{array}$ & 12,16 \\
\hline 124 & $z^{2} y+t x f_{2}\left(x^{2}, y^{3}\right)$ & $\begin{array}{c}t^{3}+z y+t x f_{2}\left(x^{2}, y^{3}\right) \subset \mathbb{P}(15,41,6,4) \\
\text { Diff }=\frac{1}{2} \Delta+\frac{3}{4} \Gamma_{2}\end{array}$ & 8 \\
\hline 125 & $z^{2} y+t x^{5}+t x^{2} y^{5}$ & $\begin{array}{c}t^{3}+z y+t x^{5}+t x^{2} y^{5} \subset \mathbb{P}(25,69,10,6) \\
\text { Diff }=\frac{1}{2} \Delta+\frac{3}{4} \Gamma_{2}\end{array}$ & 12 \\
\hline
\end{tabular}

7. Singularity $-t^{2} z+g(z, x, y)$.

\begin{tabular}{|c|c|c|c|}
\hline 1 & $\begin{array}{c}z^{i} x^{j} f_{4-i-j}(z, x)+ \\
+a x y^{4}+b z y^{4}+c t x y^{2} \\
(i \leq 1 \& c \neq 0) \&(j \leq 1 \| b \neq 0)\end{array}$ & $\begin{array}{c}t^{2} z+g\left(z, x, y^{1 / 2}\right) \subset \mathbb{P}(3,2,2,3) \\
\text { Diff }=\left(0,0,0, \frac{1}{2}\right)+\frac{2}{3} \Delta_{3}\end{array}$ & 3 \\
\hline 2 & $\begin{array}{c}z^{i} x^{j} f_{4-i-j}(z, x)+ \\
+\left(z y^{5} \| x y^{5}\right) \\
(j \leq 2) \&(i=0\|\mid\| i \leq 1)\end{array}$ & $\begin{array}{c}t z+g\left(z, x, y^{1 / 5}\right) \subset \mathbb{P}(3,1,1,3) \\
\operatorname{Diff}=\left(\frac{1}{2}, 0,0, \frac{4}{5}\right)+\frac{2}{3} \Delta_{3}\end{array}$ & 6 \\
\hline 3 & $\begin{array}{l}z^{4}+y x^{i} f_{3-i}\left(x, y^{3}\right)+ \\
\quad+\left(a z^{2} x y^{2} \| \mid a z^{2} y^{5}\right)\end{array}$ & $\begin{array}{c}t z+g(z, x, y) \subset \mathbb{P}(15,5,6,2) \\
\text { Diff }=\left(\frac{1}{2}, 0,0,0\right)+\frac{2}{3} \Delta_{4}\end{array}$ & 4 \\
\hline 4 & $\begin{array}{c}z^{4}+x^{3} y+x y^{n} \\
n=4 ; 10\end{array}$ & $\begin{array}{c}t z+g(z, x, y) \subset \mathbb{P}(9 n-3,3 n-1,4 n-4,8) \\
\text { Diff }=\left(\frac{1}{2}, 0,0,0\right)+\frac{2}{3} \Delta_{4}\end{array}$ & 7,16 \\
\hline 5 & $\begin{array}{c}z^{4}+x^{3} y+z y^{2 n} \\
n=4,5\end{array}$ & $\begin{array}{c}t^{2} z+z^{4}+x y+z y^{2 n} \subset \mathbb{P}(3 n, 2 n, 8 n-3,3) \\
\text { Diff }=\frac{2}{3} \Upsilon+\frac{8}{9} \Delta_{3}\end{array}$ & 9 \\
\hline 6 & $\begin{array}{c}z^{4}+x^{3} y+z y^{n} \\
n=5 ; 7 ; 11\end{array}$ & $\begin{array}{c}t z+z^{4}+x y+z y^{n} \subset \mathbb{P}(3 n, n, 4 n-3,3) \\
\operatorname{Diff}=\left(\frac{1}{2}, 0,0,0\right)+\frac{2}{3} \Upsilon+\frac{8}{9} \Delta_{3}\end{array}$ & $9,10,18$ \\
\hline 7 & $\begin{array}{l}z^{4}+x^{3} y+a t y^{4}+ \\
\quad+b z x y^{3}, a \neq 0\end{array}$ & $\begin{array}{c}t^{2} z+z^{4}+x^{3} y+a t y^{4}+b z x y^{3} \subset \mathbb{P}(12,8,9,5) \\
\text { Diff }=\frac{2}{3} \Delta_{4}\end{array}$ & 4 \\
\hline 8 & $z^{4}+x^{3} y+z x y^{6}$ & $\begin{array}{c}t z+z^{4}+x^{3} y+z x y^{6} \subset \mathbb{P}(51,17,21,5) \\
\text { Diff }=\left(\frac{1}{2}, 0,0,0\right)+\frac{2}{3} \Delta_{4}\end{array}$ & 10 \\
\hline 9 & $z^{4}+x^{5}+z y^{4}$ & $t^{2} z+z^{4}+x+z y^{2} \subset \mathbb{P}(3,2,8,3)$ & 15 \\
\hline
\end{tabular}




\begin{tabular}{|c|c|c|c|}
\hline & & $\mathbb{P}(1,2,1)$, Diff $=\left(0,0,0, \frac{1}{2}\right)+\frac{4}{5} \Upsilon+\frac{14}{15} \Delta_{3}$ & \\
\hline 10 & $z^{4}+x^{4} y+z y^{4}$ & $\begin{array}{c}t^{2} z+z^{4}+x y+z y^{4} \subset \mathbb{P}(6,4,13,3) \\
\text { Diff }=\frac{3}{4} \Upsilon+\frac{11}{12} \Delta_{3}\end{array}$ & 12 \\
\hline 11 & $z^{4}+x^{4} y+z x y^{3}$ & $\begin{array}{c}t z+z^{4}+x^{4} y+z x y^{3} \subset \mathbb{P}(33,11,9,8) \\
\text { Diff }=\left(\frac{1}{2}, 0,0,0\right)+\frac{2}{3} \Delta_{4}\end{array}$ & 16 \\
\hline 12 & $z^{4}+x^{3} y^{2}+x y^{5}$ & $\begin{array}{c}t z+z^{4}+x^{3} y^{2}+x y^{5} \subset \mathbb{P}(39,13,12,8) \\
\text { Diff }=\left(\frac{1}{2}, 0,0,0\right)+\frac{2}{3} \Delta_{4}\end{array}$ & 16 \\
\hline 13 & $z^{4}+\frac{x^{3} y^{2}}{+z y^{4}}+\underline{t x^{3}}+$ & $\begin{array}{c}t^{2} z+z^{4}+x y+t x+z y^{2} \subset \mathbb{P}(3,2,5,3) \\
\text { Diff }=\left(0,0,0, \frac{1}{2}\right)+\frac{2}{3} \Upsilon+\frac{8}{9} \Delta_{3}\end{array}$ & 9 \\
\hline 14 & $z^{4}+x^{3} y^{2}+z y^{5}$ & $\begin{array}{c}t z+z^{4}+x y^{2}+z y^{5} \subset \mathbb{P}(15,5,14,3) \\
\text { Diff }=\left(\frac{1}{2}, 0,0,0\right)+\frac{2}{3} \Upsilon+\frac{8}{9} \Delta_{3}\end{array}$ & 18 \\
\hline 15 & $z^{4}+x^{3} y^{2}+z x y^{3}$ & $\begin{array}{c}t z+z^{4}+x^{3} y^{2}+z x y^{3} \subset \mathbb{P}(21,7,6,5) \\
\text { Diff }=\left(\frac{1}{2}, 0,0,0\right)+\frac{2}{3} \Delta_{4}\end{array}$ & 10 \\
\hline 16 & $z^{4}+t x^{i} f_{3-i}(x, y)$ & $\begin{array}{c}t^{2} z+z^{4}+t x^{i} f_{3-i}(x, y) \subset \mathbb{P}(9,6,5,5) \\
\text { Diff }=\frac{4}{5} \Gamma_{2}\end{array}$ & 5 \\
\hline 17 & $z^{4}+t x^{2} y+x y^{4}$ & $\begin{array}{c}t^{2} z+z^{4}+t x^{2} y+x y^{4} \subset \mathbb{P}(21,14,12,11) \\
\text { Diff }=\frac{2}{3} \Delta_{4}\end{array}$ & 6 \\
\hline 18 & $\begin{array}{l}z^{4}+t x^{2} y+a z y^{4}+ \\
+b x^{2} y^{3}\end{array}$ & $\begin{array}{c}t^{2} z+z^{4}+t x y+a z y^{4}+b x y^{3} \subset \mathbb{P}(6,4,7,3) \\
\text { Diff }=\frac{1}{2} \Upsilon+\frac{5}{6} \Delta_{3}\end{array}$ & 6 \\
\hline 19 & $z^{4}+t x^{2} y+z y^{5}$ & $\begin{array}{c}t^{2} z+z^{4}+t x y+z y^{5} \subset \mathbb{P}(15,10,19,6) \\
\text { Diff }=\frac{1}{2} \Upsilon+\frac{5}{6} \Delta_{3}\end{array}$ & 12 \\
\hline 20 & $z^{4}+t x^{2} y+z x y^{3}$ & $\begin{array}{c}t^{2} z+z^{4}+t x^{2} y+z x y^{3} \subset \mathbb{P}(15,10,9,7) \\
\text { Diff }=\frac{2}{3} \Delta_{4}\end{array}$ & 7 \\
\hline 21 & $z^{4}+t x^{2} y+t y^{4}$ & $\begin{array}{c}t^{2} z+z^{4}+t x y+t y^{4} \subset \mathbb{P}(12,8,15,5) \\
\text { Diff }=\left(0,0, \frac{1}{2}, 0\right)+\frac{4}{5} \Gamma_{2}+\frac{2}{3} \Delta_{4}\end{array}$ & 10 \\
\hline 22 & $\begin{array}{c}z^{3} x+x^{3} y+a x^{2} y^{3}+ \\
+b x y^{5}+c y^{7},|b|+|c| \neq 0\end{array}$ & $\begin{array}{c}t^{2} z+g(z, x, y) \subset \mathbb{P}(8,5,6,3) \\
\text { Diff }=\frac{1}{2} \Delta_{4}\end{array}$ & 3 \\
\hline 23 & $\begin{array}{c}z^{3} x+x^{3} y+x y^{n} \\
n=4 ; 8\end{array}$ & $\begin{array}{c}t z+g(z, x, y) \subset \mathbb{P}(7 n-3,2 n, 3 n-3,6) \\
\text { Diff }=\frac{1}{2} \Gamma+\frac{3}{4} \Gamma_{3}\end{array}$ & 5,12 \\
\hline 24 & $\begin{array}{l}z^{3} x+x^{3} y+a z^{2} x y^{2}+ \\
\quad+b z x y^{4}+c x y^{6}\end{array}$ & $\begin{array}{c}t z+g(z, x, y) \subset \mathbb{P}(13,4,5,2) \\
\text { Diff }=\frac{1}{2} \Gamma+\frac{3}{4} \Gamma_{3}\end{array}$ & 4 \\
\hline 25 & $\begin{array}{c}z^{3} x+x^{3} y+z y^{2 n} \\
n=3,4\end{array}$ & $\begin{array}{c}t^{2} z+g(z, x, y) \subset \mathbb{P}(7 n, 4 n+1,6 n-2,7) \\
\text { Diff }=\frac{6}{7} \Delta_{3}+\frac{n-3}{n-2} \Delta_{4}\end{array}$ & 7 \\
\hline 26 & $\begin{array}{c}z^{3} x+x^{3} y+z y^{n} \\
n=5 ; 7 ; 9\end{array}$ & $\begin{array}{c}t z+g(z, x, y) \subset \mathbb{P}(7 n, 2 n+1,3 n-2,7) \\
\text { Diff }=\left(\frac{1}{2}, 0,0,0\right)+\frac{6}{7} \Delta_{3}\end{array}$ & $7,10,14$ \\
\hline 27 & $z^{3} x+x^{3} y+z x y^{5}$ & $\begin{array}{c}t z+z^{3} x+x^{3} y+z x y^{5} \subset \mathbb{P}(33,10,13,4) \\
\text { Diff }=\frac{1}{2} \Gamma+\frac{3}{4} \Gamma_{3}\end{array}$ & 8 \\
\hline 28 & $\begin{array}{l}z^{3} x+x^{3} y+t y^{n} \\
\quad n=3 ; 5 ; 7\end{array}$ & $\begin{array}{c}t^{2} z+g(z, x, y) \subset \mathbb{P}(7 n-1,4 n+1,6 n-4,11) \\
\text { Diff }=\frac{1}{2} \Delta_{4}\end{array}$ & $4,7,11$ \\
\hline 29 & $z^{3} x+x^{5}+z y^{4}$ & $\begin{array}{c}t^{2} z+z^{3} x+x^{5}+z y^{2} \subset \mathbb{P}(11,8,6,11) \\
\text { Diff }=\left(0,0,0, \frac{1}{2}\right)+\frac{10}{11} \Delta_{3}\end{array}$ & 11 \\
\hline 30 & $z^{3} x+x^{5}+z x y^{3}$ & $\begin{array}{c}t z+z^{3} x+x^{5}+z x y \subset \mathbb{P}(11,4,3,8) \\
\text { Diff }=\left(0,0,0, \frac{2}{3}\right)+\frac{1}{2} \Gamma+\frac{7}{8} \Gamma_{3}\end{array}$ & 16 \\
\hline 31 & $z^{3} x+x^{4} y+z y^{4}$ & $t^{2} z+z^{3} x+x^{4} y+z y^{4} \subset \mathbb{P}(18,13,10,9)$ & 9 \\
\hline
\end{tabular}




\begin{tabular}{|c|c|c|c|}
\hline & & Diff $=\frac{8}{9} \Delta_{3}+\frac{1}{2} \Delta_{4}$ & \\
\hline 32 & $z^{3} x+x^{4} y+z x y^{3}$ & $\begin{array}{c}t z+z^{3} x+x^{4} y+z x y^{3} \subset \mathbb{P}(25,9,7,6) \\
\text { Diff }=\frac{1}{2} \Gamma+\frac{5}{6} \Gamma_{3}\end{array}$ & 12 \\
\hline 33 & $z^{3} x+x^{3} y^{2}+y^{6}$ & $\begin{array}{c}t^{2} z+z^{3} x+x^{3} y+y^{3} \subset \mathbb{P}(10,7,6,9) \\
\text { Diff }=\frac{1}{2} \Omega+\frac{3}{4} \Delta_{4}\end{array}$ & 9 \\
\hline 34 & $z^{3} x+x^{3} y^{2}+x y^{5}$ & $\begin{array}{c}t z+z^{3} x+x^{3} y^{2}+x y^{5} \subset \mathbb{P}(29,10,9,6) \\
\text { Diff }=\frac{1}{2} \Gamma+\frac{3}{4} \Gamma_{3}\end{array}$ & 12 \\
\hline 35 & $z^{3} x+\frac{x^{3} y^{2}}{+z y^{4}}+\underline{t x^{3}}+$ & $\begin{array}{c}t^{2} z+z^{3} x+\underline{x^{3} y}+\underline{t x^{3}}+z y^{2} \subset \mathbb{P}(7,5,4,7) \\
\text { Diff }=\left(0,0,0, \frac{1}{2}\right)+\frac{6}{7} \Delta_{3}\end{array}$ & 7 \\
\hline 36 & $z^{3} x+x^{3} y^{2}+z y^{5}$ & $\begin{array}{c}t z+z^{3} x+x^{3} y^{2}+z y^{5} \subset \mathbb{P}(35,12,11,7) \\
\operatorname{Diff}=\left(\frac{1}{2}, 0,0,0\right)+\frac{6}{7} \Delta_{3}\end{array}$ & 14 \\
\hline 37 & $z^{3} x+x^{3} y^{2}+z x y^{3}$ & $\begin{array}{c}t z+z^{3} x+x^{3} y^{2}+z x y^{3} \subset \mathbb{P}(17,6,5,4) \\
\text { Diff }=\frac{1}{2} \Gamma+\frac{3}{4} \Gamma_{3}\end{array}$ & 8 \\
\hline 38 & $z^{3} x+t x^{3}+x y^{4}$ & $\begin{array}{c}t^{2} z+z^{3} x+t x^{3}+x y \subset \mathbb{P}(7,5,4,15) \\
\text { Diff }=\left(0,0,0, \frac{3}{4}\right)+\frac{4}{5} \Gamma_{3}\end{array}$ & 5 \\
\hline 39 & $z^{3} x+t x^{3}+z x y^{3}$ & $\begin{array}{c}t^{2} z+z^{3} x+t x^{3}+z x y \subset \mathbb{P}(7,5,4,10) \\
\text { Diff }=\left(0,0,0, \frac{2}{3}\right)+\frac{1}{2} \Gamma_{2}+\frac{4}{5} \Gamma_{3}\end{array}$ & 10 \\
\hline 40 & $\begin{array}{c}z^{3} x+t x^{i} y^{j} f_{3-i-j}(x, y) \\
i=0 \& j \leq 1\end{array}$ & $\begin{array}{c}t^{2} z+g(z, x, y) \subset \mathbb{P}(7,5,4,4) \\
\text { Diff }=\frac{3}{4} \Gamma_{2}\end{array}$ & 4 \\
\hline 41 & $\begin{array}{c}z^{3} x+t x^{2} y+y^{n} \\
n=5 ; 7\end{array}$ & $\begin{aligned} t^{2} z+g(z, x, y) \subset & \mathbb{P}(5 n-1,3 n+2,4 n-6,13) \\
& \operatorname{Diff}=\frac{1}{2} \Delta_{4}\end{aligned}$ & 9,13 \\
\hline 42 & $\begin{array}{c}z^{3} x+t x^{2} y+x y^{n} \\
n=4 ; 5\end{array}$ & $\begin{array}{c}t^{2} z+g(z, x, y) \subset \mathbb{P}(5 n-3,3 n, 4 n-6,9) \\
\text { Diff }=\frac{2}{3} \Gamma_{3}+\frac{n-4}{n-3} \Delta_{4}\end{array}$ & 5,9 \\
\hline 43 & $\begin{array}{l}z^{3} x+t x^{2} y+a z y^{4}+ \\
\quad+b x^{2} y^{3}, a \neq 0\end{array}$ & $\begin{array}{c}t^{2} z+g(z, x, y) \subset \mathbb{P}(10,7,6,5) \\
\operatorname{Diff}=\frac{4}{5} \Delta_{3}+\frac{1}{2} \Delta_{4}\end{array}$ & 5 \\
\hline 44 & $z^{3} x+t x^{2} y+z y^{5}$ & $\begin{array}{c}t^{2} z+g(z, x, y) \subset \mathbb{P}(25,17,16,10) \\
\text { Diff }=\frac{4}{5} \Delta_{3}+\frac{1}{2} \Gamma_{2}\end{array}$ & 10 \\
\hline 45 & $z^{3} x+t x^{2} y+z x y^{3}$ & $\begin{array}{c}t^{2} z+g(z, x, y) \subset \mathbb{P}(13,9,8,6) \\
\text { Diff }=\frac{2}{3} \Gamma_{3}+\frac{1}{2} \Gamma_{2}\end{array}$ & 6 \\
\hline 46 & $z^{3} x+t x^{2} y+z^{2} y^{3}$ & $\begin{array}{c}t^{2} z+g(z, x, y) \subset \mathbb{P}(16,11,10,7) \\
\text { Diff }=\frac{1}{2} \Delta_{4}\end{array}$ & 7 \\
\hline 47 & $z^{3} x+t x^{2} y+t y^{4}$ & $\begin{array}{c}t^{2} z+g(z, x, y) \subset \mathbb{P}(19,13,12,8) \\
\text { Diff }=\frac{3}{4} \Gamma_{2}\end{array}$ & 8 \\
\hline 48 & $z^{3} y+x^{5}+z y^{4}$ & $\begin{array}{c}t^{2} z+z^{3} y+x+z y^{4} \subset \mathbb{P}(4,3,11,2) \\
\mathbb{P}(2,3,1), \text { Diff }=\frac{4}{5} \Upsilon+\frac{9}{10} \Delta_{3}\end{array}$ & 10 \\
\hline 49 & $z^{3} y+x^{4} y+y^{6}$ & $\begin{array}{c}t^{2} z+z^{3} y+x^{2} y+y^{6} \subset \mathbb{P}(13,10,15,6) \\
\text { Diff }=\left(0,0, \frac{1}{2}, 0\right)+\frac{4}{5} \Gamma_{4}\end{array}$ & 12 \\
\hline 50 & $z^{3} y+x^{4} y+x y^{5}$ & $\begin{array}{c}t z+g(z, x, y) \subset \mathbb{P}(41,16,12,9) \\
\text { Diff }=\frac{1}{2} \Gamma+\frac{7}{8} \Gamma_{4}\end{array}$ & 18 \\
\hline 51 & $z^{3} y+x^{4} y+z y^{4}$ & $\begin{array}{c}t^{2} z+z^{3} y+x y+z y^{4} \subset \mathbb{P}(4,3,9,2) \\
\text { Diff }=\frac{3}{4} \Upsilon+\frac{7}{8} \Delta_{3}+\frac{2}{3} \Gamma_{4}\end{array}$ & 8 \\
\hline 52 & $z^{3} y+x^{4} y+z x y^{3}$ & $\begin{array}{c}t z+g(z, x, y) \subset \mathbb{P}(21,8,6,5) \\
\text { Diff }=\frac{1}{2} \Gamma+\frac{3}{4} \Gamma_{4}+\frac{2}{3} \Delta_{4}\end{array}$ & 10 \\
\hline 53 & $z^{3} y+x^{4} y+t y^{4}$ & $t^{2} z+z^{3} y+x y+t y^{4} \subset \mathbb{P}(9,7,21,4)$ & 16 \\
\hline
\end{tabular}




\begin{tabular}{|c|c|c|c|}
\hline & & Diff $=\left(0,0, \frac{3}{4}, 0\right)+\frac{6}{7} \Gamma_{4}+\frac{2}{3} \Delta_{4}$ & \\
\hline 54 & $z^{3} y+z x^{3}+y^{8}$ & $\begin{array}{c}t z+z^{3} y+z x+y^{8} \subset \mathbb{P}(17,7,17,3) \\
\text { Diff }=\left(\frac{1}{2}, 0, \frac{2}{3}, 0\right)+\frac{16}{17} \Delta_{4}\end{array}$ & 18 \\
\hline 55 & $\begin{array}{l}z^{3} y+z x^{3}+a z^{2} y^{3}+ \\
b z y^{5}+c x^{3} y^{2}+d y^{7}\end{array}$ & $\begin{array}{c}t z+g\left(z, x^{1 / 3}, y\right) \subset \mathbb{P}(5,2,5,1) \\
\text { Diff }=\left(\frac{1}{2}, 0, \frac{2}{3}, 0\right)+\frac{4}{5} \Delta_{4}\end{array}$ & 6 \\
\hline 56 & $\begin{array}{c}z^{3} y+z x^{3}+x y^{n} \\
n=5 ; 6\end{array}$ & $\begin{array}{c}t z+g(z, x, y) \subset \mathbb{P}(6 n+3,3 n-2,2 n+1,7) \\
\text { Diff }=\left(\frac{1}{2}, 0,0,0\right)+\frac{2 n}{2 n+1} \Delta_{4}\end{array}$ & 11,14 \\
\hline 57 & $\begin{array}{c}z^{3} y+z x^{3}+x^{2} y^{n} \\
n=3 ; 4\end{array}$ & $\begin{array}{c}t z+g(z, x, y) \subset \mathbb{P}(6 n+3,3 n-1,2 n+1,5) \\
\text { Diff }=\left(\frac{1}{2}, 0,0,0\right)+\frac{2 n}{2 n+1} \Delta_{4}\end{array}$ & 7,10 \\
\hline 58 & $\begin{array}{c}z^{3} y+z x^{3}+a t x^{2} y+ \\
b t y^{4}+c z x y^{3}\end{array}$ & $\begin{array}{c}t^{2} z+g(z, x, y) \subset \mathbb{P}(9,7,6,4) \\
\text { Diff }=\frac{1}{2} \Gamma_{2}+\frac{2}{3} \Delta_{4}\end{array}$ & 4 \\
\hline 59 & $z^{3} y+z x^{3}+t y^{5}$ & $\begin{array}{c}t^{2} z+z^{3} y+z x+t y^{5} \subset \mathbb{P}(11,9,22,4) \\
\text { Diff }=\left(0,0, \frac{2}{3}, 0\right)+\frac{1}{2} \Gamma_{2}+\frac{10}{11} \Delta_{4}\end{array}$ & 12 \\
\hline 60 & $\begin{array}{c}z^{3} y+z x^{3}+t x y^{n} \\
n=2 ; 3\end{array}$ & $\begin{array}{c}t^{2} z+g(z, x, y) \subset \mathbb{P}(6 n+3,6 n-1,4 n+2,8) \\
\text { Diff }=\frac{1}{2} \Gamma_{2}+\frac{2 n}{2 n+1} \Delta_{4}\end{array}$ & 5,8 \\
\hline 61 & $\begin{array}{l}z^{3} y+z^{2} x^{2}+a x^{2} y^{3}+ \\
+b z y^{4}+c t x^{2} y, c \neq 0\end{array}$ & $\begin{array}{c}t^{2} z+g\left(z, x^{1 / 2}, y\right) \subset \mathbb{P}(4,3,5,2) \\
\text { Diff }=\frac{1}{2} \Upsilon+\frac{3}{4} \Delta_{3}\end{array}$ & 4 \\
\hline 62 & $\begin{array}{c}z^{3} y+t x^{3}+x y^{n} \\
n=4 ; 5\end{array}$ & $\begin{aligned} t^{2} z+g(z, x, y) \subset & \mathbb{P}(6 n+2,6 n-5,4 n-1,14) \\
& \operatorname{Diff}=\frac{1}{2} \Delta_{3}\end{aligned}$ & 5,14 \\
\hline 63 & $\begin{array}{c}z^{3} y+t x^{3}+z y^{4}+ \\
+a x^{3} y^{2}\end{array}$ & $\begin{array}{c}t^{2} z+g\left(z, x^{1 / 3}, y\right) \subset \mathbb{P}(4,3,7,2) \\
\text { Diff }=\frac{2}{3} \Upsilon+\frac{5}{6} \Delta_{3}\end{array}$ & 6 \\
\hline 64 & $z^{3} y+t x^{3}+z x y^{3}$ & $\begin{array}{c}t^{2} z+g(z, x, y) \subset \mathbb{P}(17,13,10,8) \\
\text { Diff }=\frac{1}{2} \Gamma_{2}\end{array}$ & 8 \\
\hline 65 & $z^{3} y+t x^{3}+t y^{4}$ & $\begin{array}{c}t^{2} z+z^{3} y+t x+t y^{4} \subset \mathbb{P}(9,7,16,4) \\
\text { Diff }=\left(0,0, \frac{2}{3}, 0\right)+\frac{3}{4} \Gamma_{2}\end{array}$ & 12 \\
\hline 66 & $z^{2} x^{2}+x^{5}+z y^{4}$ & $\begin{array}{c}t^{2} z+z^{2} x^{2}+x^{5}+z y^{2} \subset \mathbb{P}(7,6,4,7) \\
\text { Diff }=\left(0,0,0, \frac{1}{2}\right)+\frac{6}{7} \Delta_{3}\end{array}$ & 7 \\
\hline 67 & $z^{2} x^{2}+x^{4} y+x y^{5}$ & $\begin{array}{c}t z+z^{2} x^{2}+x^{4} y+x y^{5} \subset \mathbb{P}(27,11,8,6) \\
\text { Diff }=\left(\frac{1}{2}, 0,0,0\right)+\frac{2}{3} \Delta_{3}\end{array}$ & 12 \\
\hline 68 & $z^{2} x^{2}+x^{4} y+z y^{4}$ & $\begin{array}{c}t^{2} z+z^{2} x+x^{2} y+z y^{4} \subset \mathbb{P}(6,5,7,3) \\
\text { Diff }=\frac{1}{2} \Upsilon+\frac{5}{6} \Delta_{3}\end{array}$ & 6 \\
\hline 69 & $\begin{array}{c}z^{2} x^{2}+x^{3} y^{2}+a t x^{3}+ \\
+z y^{4}\end{array}$ & $\begin{array}{c}t^{2} z+z^{2} x^{2}+x^{3} y+a t x^{3}+z y^{2} \subset \mathbb{P}(5,4,3,5) \\
\text { Diff }=\left(0,0,0, \frac{1}{2}\right)+\frac{4}{5} \Delta_{3}\end{array}$ & 5 \\
\hline 70 & $z^{2} x^{2}+x^{3} y^{2}+z y^{5}$ & $\begin{array}{c}t z+z^{2} x^{2}+x^{3} y^{2}+z y^{5} \subset \mathbb{P}(25,9,8,5) \\
\text { Diff }=\left(\frac{1}{2}, 0,0,0\right)+\frac{4}{5} \Delta_{3}\end{array}$ & 10 \\
\hline 71 & $z^{2} x^{2}+t x^{2} y+z y^{5}$ & $\begin{array}{c}t^{2} z+z^{2} x+t x y+z y^{5} \subset \mathbb{P}(10,7,13,4) \\
\text { Diff }=\frac{1}{2} \Upsilon+\frac{3}{4} \Delta_{3}\end{array}$ & 8 \\
\hline 72 & $\begin{array}{c}z^{2} x^{2}+t x^{2} y+a t y^{4}+ \\
+b z^{2} y^{3}\end{array}$ & $\begin{array}{c}t^{2} z+g\left(z, x^{1 / 2}, y\right) \subset \mathbb{P}(7,5,9,3) \\
\operatorname{Diff}=\left(0,0, \frac{1}{2}, 0\right)+\frac{2}{3} \Gamma_{2}\end{array}$ & 6 \\
\hline 73 & $\begin{array}{c}z^{2} x^{2}+t x^{i} y^{j} f_{3-i-j}(x, y) \\
i=0 \& j \leq 1\end{array}$ & $\begin{array}{c}t^{2} z+g(z, x, y) \subset \mathbb{P}(5,4,3,3) \\
\text { Diff }=\frac{2}{3} \Gamma_{2}\end{array}$ & 3 \\
\hline 74 & $\begin{array}{c}z^{2} x y+t x^{i}(x+y)^{j} \\
f_{3-i-j}(x, y), i=0 \& j \leq 1\end{array}$ & $\begin{array}{c}t^{2} z+g(z, x, y) \subset \mathbb{P}(5,4,3,3) \\
\text { Diff }=\frac{2}{3} \Gamma_{2}\end{array}$ & 3 \\
\hline 75 & $z^{2} x y+x^{5}+a z x y^{3}+$ & $t z+g(z, x, y) \subset \mathbb{P}(17,8,5,4)$ & 8 \\
\hline
\end{tabular}




\begin{tabular}{|c|c|c|c|}
\hline & $+b x y^{5}$ & Diff $=\frac{1}{2} \Gamma+\frac{7}{8} \Gamma_{3}$ & \\
\hline 76 & $z^{2} x y+x^{5}+x^{2} y^{4}$ & $\begin{array}{c}t z+z^{2} x y+x^{5}+x^{2} y^{4} \subset \mathbb{P}(27,13,8,6) \\
\text { Diff }=\left(\frac{1}{2}, 0,0,0\right)+\frac{2}{3} \Delta_{3}\end{array}$ & 12 \\
\hline 77 & $z^{2} x y+x^{5}+z y^{4}$ & $\begin{array}{c}t^{2} z+g(z, x, y) \subset \mathbb{P}(12,11,7,6) \\
\text { Diff }=\frac{5}{6} \Delta_{3}\end{array}$ & 6 \\
\hline 78 & $z^{2} x y+x^{5}+t x y^{3}$ & $\begin{array}{c}t^{2} z+g(z, x, y) \subset \mathbb{P}(22,21,13,10) \\
\text { Diff }=\frac{1}{2} \Delta_{3}\end{array}$ & 10 \\
\hline 79 & $\begin{array}{c}z^{2} x y+x^{4} y+y^{n} \\
n=6 ; 8\end{array}$ & $\begin{array}{c}t z+g(z, x, y) \subset \mathbb{P}(5 n+3,3 n-3,2 n-2,8) \\
\text { Diff }=\frac{1}{2} \Gamma+\frac{2 n-3}{2 n-2} \Gamma_{4}\end{array}$ & 10,16 \\
\hline 80 & $\begin{array}{c}z^{2} x y+x^{4} y+a x^{2} y^{4}+ \\
+b y^{7}\end{array}$ & $\begin{array}{c}t z+g(z, x, y) \subset \mathbb{P}(19,9,6,4) \\
\text { Diff }=\frac{1}{2} \Gamma+\frac{5}{6} \Gamma_{4}\end{array}$ & 8 \\
\hline 81 & $\begin{aligned} z^{2} x y+ & x^{4} y+a z x y^{3}+ \\
& +b x y^{5}\end{aligned}$ & $\begin{array}{c}t z+g(z, x, y) \subset \mathbb{P}(13,6,4,3) \\
\text { Diff }=\frac{1}{2} \Gamma+\frac{3}{4} \Gamma_{4}+\frac{5}{6} \Gamma_{3}\end{array}$ & 6 \\
\hline 82 & $z^{2} x y+x^{4} y+x y^{6}$ & $\begin{array}{c}t z+g(z, x, y) \subset \mathbb{P}(31,15,10,6) \\
\text { Diff }=\frac{1}{2} \Gamma+\frac{9}{10} \Gamma_{4}+\frac{5}{6} \Gamma_{3}\end{array}$ & 12 \\
\hline 83 & $z^{2} x y+x^{4} y+z y^{4}$ & $\begin{array}{c}t^{2} z+g(z, x, y) \subset \mathbb{P}(10,9,6,5) \\
\text { Diff }=\frac{4}{5} \Delta_{3}+\frac{2}{3} \Gamma_{4}+\frac{1}{2} \Delta_{4}\end{array}$ & 5 \\
\hline 84 & $z^{2} x y+x^{4} y+z y^{5}$ & $\begin{array}{c}t z+g(z, x, y) \subset \mathbb{P}(25,12,8,5) \\
\text { Diff }=\frac{1}{2} \Gamma+\frac{7}{8} \Gamma_{4}+\frac{4}{5} \Delta_{3}\end{array}$ & 10 \\
\hline 85 & $\begin{array}{c}z^{2} x y+x^{4} y+t y^{n} \\
n=4 ; 5\end{array}$ & $\begin{array}{c}t^{2} z+g(z, x, y) \subset \mathbb{P}(5 n+3,6 n-3,4 n-2,11) \\
\text { Diff }=\frac{2 n-2}{2 n-1} \Gamma_{4}+\frac{n-4}{n-3} \Delta_{4}\end{array}$ & 7,11 \\
\hline 86 & $z^{2} x y+x^{4} y+t x y^{3}$ & $\begin{array}{c}t^{2} z+g(z, x, y) \subset \mathbb{P}(16,15,10,7) \\
\text { Diff }=\frac{4}{5} \Gamma_{4}+\frac{1}{2} \Delta_{4}\end{array}$ & 7 \\
\hline 87 & $\begin{array}{l}z^{2} x+x^{4}+a t x^{2} y^{2}+ \\
\quad+b z x y^{4}+c x y^{8}\end{array}$ & $\begin{array}{c}t^{2} z+g\left(z, x, y^{1 / 2}\right) \subset \mathbb{P}(5,6,4,3) \\
\text { Diff }=\left(0,0,0, \frac{1}{2}\right)+\frac{2}{3} \Gamma_{3}\end{array}$ & 3 \\
\hline 88 & $\begin{array}{l}z^{2} x+x^{4}+x y^{n} \\
n=5 ; 7 ; 11\end{array}$ & $\begin{array}{c}t z+z^{2} x+x^{4}+x y \subset \mathbb{P}(5,3,2,6) \\
\text { Diff }=\left(0,0,0, \frac{n-1}{n}\right)+\frac{1}{2} \Gamma+\frac{5}{6} \Gamma_{3}\end{array}$ & $6,7,12$ \\
\hline 89 & $\begin{aligned} z^{2} x+ & x^{4}+a z x y^{5}+ \\
& +b x y^{10}\end{aligned}$ & $\begin{array}{c}t z+z^{2} x+x^{4}+a z x y+b x y^{2} \subset \mathbb{P}(5,3,2,3) \\
\text { Diff }=\left(0,0,0, \frac{4}{5}\right)+\frac{1}{2} \Gamma+\frac{5}{6} \Gamma_{3}\end{array}$ & 6 \\
\hline 90 & $\begin{array}{c}z^{2} x+x^{4}+z y^{n} \\
n=7,9\end{array}$ & $\begin{array}{c}t z+z^{2} x+x^{4}+z y \subset \mathbb{P}(5,3,2,5) \\
\text { Diff }=\left(\frac{1}{2}, 0,0, \frac{n-1}{n}\right)+\frac{4}{5} \Delta_{3}\end{array}$ & 10 \\
\hline 91 & $\begin{array}{c}z^{2} x+x^{4}+z y^{2 n} \\
n=3,4\end{array}$ & $\begin{array}{c}t^{2} z+z^{2} x+x^{4}+z y^{2} \subset \mathbb{P}(5,6,4,5) \\
\text { Diff }=\left(0,0,0, \frac{n-1}{n}\right)+\frac{4}{5} \Delta_{3}\end{array}$ & 5 \\
\hline 92 & $\frac{z^{2} x+z x^{3}+x^{5}+}{x y^{n}, n=5 ; 7}$ & $\begin{array}{c}t z+\frac{z^{2} x+z x^{3}+x^{5}+x y \subset \mathbb{P}(3,2,1,4)}{\text { Diff }=\left(0,0,0, \frac{n-1}{n}\right)+\frac{1}{2} \Gamma+\frac{3}{4} \Gamma_{3}}\end{array}$ & 5,8 \\
\hline 93 & $\frac{z^{2} x+z x^{3}+x^{5}+}{+a x^{3} y^{3}+x y^{6}}$ & $\begin{array}{c}t z+g\left(z, x, y^{1 / 3}\right) \subset \mathbb{P}(3,2,1,2) \\
\text { Diff }=\left(0,0,0, \frac{2}{3}\right)+\frac{1}{2} \Gamma+\frac{3}{4} \Gamma_{3}\end{array}$ & 4 \\
\hline 94 & $\begin{array}{l}\frac{z^{2} x+z x^{3}+x^{5}+a t x^{2} y^{2}}{+b z y^{4}+c x^{2} y^{4}, b \neq 0}\end{array}$ & $\begin{array}{c}t^{2} z+g\left(z, x, y^{1 / 2}\right) \subset \mathbb{P}(3,4,2,3) \\
\text { Diff }=\left(0,0,0, \frac{1}{2}\right)+\frac{2}{3} \Delta_{3}\end{array}$ & 3 \\
\hline 95 & $\frac{z^{2} x+z x^{3}+x^{5}+}{+a z y^{5}+b x^{2} y^{5}}$ & $\begin{array}{c}t z+g\left(z, x, y^{1 / 5}\right) \subset \mathbb{P}(3,2,1,3) \\
\text { Diff }=\left(\frac{1}{2}, 0,0, \frac{4}{5}\right)+\frac{2}{3} \Delta_{3}\end{array}$ & 6 \\
\hline 96 & $\begin{array}{c}z^{2} x+x^{4} y+x y^{n} \\
n=5 ; 9\end{array}$ & $\begin{array}{c}t z+g(z, x, y) \subset \mathbb{P}(5 n-2,3 n, 2 n-2,6) \\
\text { Diff }=\frac{1}{2} \Gamma+\frac{5}{6} \Gamma_{3}\end{array}$ & 6,12 \\
\hline 97 & $z^{2} x+x^{4} y+a t x^{2} y^{2}+$ & $t^{2} z+g(z, x, y) \subset \mathbb{P}(7,9,5,3)$ & 3 \\
\hline
\end{tabular}




\begin{tabular}{|c|c|c|c|}
\hline & $+b z x y^{3}+c x y^{6}$ & Diff $=\frac{2}{3} \Gamma_{3}$ & \\
\hline 98 & $\begin{array}{c}z^{2} x+x^{4} y+a z x y^{4}+ \\
+b x y^{8}\end{array}$ & $\begin{array}{c}t z+g(z, x, y) \subset \mathbb{P}(19,12,7,3) \\
\text { Diff }=\frac{1}{2} \Gamma+\frac{5}{6} \Gamma_{3}\end{array}$ & 6 \\
\hline 99 & $\begin{array}{c}z^{2} x+x^{4} y+z y^{2 n} \\
n=2,3\end{array}$ & $\begin{array}{c}t^{2} z+g(z, x, y) \subset \mathbb{P}(5 n, 6 n+1,4 n-1,5) \\
\text { Diff }=\frac{4}{5} \Delta_{3}\end{array}$ & 5 \\
\hline 100 & $\begin{array}{c}z^{2} x+x^{4} y+z y^{n} \\
n=5 ; 7\end{array}$ & $\begin{array}{c}t z+g(z, x, y) \subset \mathbb{P}(5 n, 3 n+1,2 n-1,5) \\
\text { Diff }=\left(\frac{1}{2}, 0,0,0\right)+\frac{4}{5} \Delta_{3}\end{array}$ & 6,10 \\
\hline 101 & $\begin{aligned} z^{2} x+ & x^{6}+a z x y^{3}+ \\
& +b x y^{6}\end{aligned}$ & $\begin{array}{c}t z+z^{2} x+x^{6}+a z x y+b x y^{2} \subset \mathbb{P}(7,5,2,5) \\
\text { Diff }=\left(0,0,0, \frac{2}{3}\right)+\frac{1}{2} \Gamma+\frac{9}{10} \Gamma_{3}\end{array}$ & 10 \\
\hline 102 & $z^{2} x+x^{6}+z y^{4}$ & $\begin{array}{c}t^{2} z+z^{2} x+x^{6}+z y^{2} \subset \mathbb{P}(7,10,4,7) \\
\text { Diff }=\left(0,0,0, \frac{1}{2}\right)+\frac{6}{7} \Delta_{3}\end{array}$ & 7 \\
\hline 103 & $\begin{array}{c}z^{2} x+x^{5} y+a z x y^{3}+ \\
+b x y^{6}\end{array}$ & $\begin{array}{c}t z+g(z, x, y) \subset \mathbb{P}(17,12,5,4) \\
\text { Diff }=\frac{1}{2} \Gamma+\frac{7}{8} \Gamma_{3}\end{array}$ & 8 \\
\hline 104 & $z^{2} x+x^{5} y+x^{2} y^{5}$ & $\begin{array}{c}t z+z^{2} x+x^{5} y+x^{2} y^{5} \subset \mathbb{P}(27,19,8,6) \\
\text { Diff }=\left(\frac{1}{2}, 0,0,0\right)+\frac{2}{3} \Delta_{3}\end{array}$ & 12 \\
\hline 105 & $z^{2} x+x^{5} y+z y^{4}$ & $\begin{array}{c}t^{2} z+z^{2} x+x^{5} y+z y^{4} \subset \mathbb{P}(12,17,7,6) \\
\text { Diff }=\frac{5}{6} \Delta_{3}\end{array}$ & 6 \\
\hline 106 & $z^{2} x+x^{5} y+t x y^{4}$ & $\begin{array}{c}t^{2} z+z^{2} x+x^{5} y+t x y^{4} \subset \mathbb{P}(22,31,13,10) \\
\text { Diff }=\frac{1}{2} \Delta_{3}\end{array}$ & 10 \\
\hline 107 & $\begin{array}{c}z^{2} x+x^{4} y^{2}+a z x y^{3}+ \\
+b x y^{6}\end{array}$ & $\begin{array}{c}z+g(z, x, y) \subset \mathbb{P}(13,9,4,3) \\
\text { Diff }=\frac{1}{2} \Gamma+\frac{5}{6} \Gamma_{3}\end{array}$ & 6 \\
\hline 108 & $z^{2} x+x^{4} y^{2}+x y^{7}$ & $\begin{array}{c}t z+z^{2} x+x^{4} y^{2}+x y^{7} \subset \mathbb{P}(31,21,10,6) \\
\text { Diff }=\frac{1}{2} \Gamma+\frac{5}{6} \Gamma_{3}\end{array}$ & 12 \\
\hline 109 & $z^{2} x+\frac{x^{4} y^{2}}{+z y^{4}}+\underline{t x^{4}}+$ & $\begin{array}{c}t^{2} z+z^{2} x+x^{4} y+t \underline{t x^{4}}+z y^{2} \subset \mathbb{P}(5,7,3,5) \\
\text { Diff }=\left(0,0,0, \frac{1}{2}\right)+\frac{4}{5} \Delta_{3}\end{array}$ & 5 \\
\hline 110 & $z^{2} x+x^{4} y^{2}+z y^{5}$ & $\begin{array}{c}t z+z^{2} x+x^{4} y^{2}+z y^{5} \subset \mathbb{P}(25,17,8,5) \\
\text { Diff }=\left(\frac{1}{2}, 0,0,0\right)+\frac{4}{5} \Delta_{3}\end{array}$ & 10 \\
\hline 111 & $\begin{array}{l}z^{2} x+t x^{3}+a z x y^{n}+ \\
\quad+b x y^{2 n}, n=3,4\end{array}$ & $\begin{array}{c}t^{2} z+z^{2} x+t x^{3}+a z x y+b x y^{2} \subset \mathbb{P}(4,5,3,5) \\
\text { Diff }=\left(0,0,0, \frac{n-1}{n}\right)+\frac{4}{5} \Gamma_{3}\end{array}$ & 5 \\
\hline 112 & $\begin{array}{c}z^{2} x+t x^{3}+a t x y^{3}+ \\
b z x^{2} y+c z y^{4}+d x^{3} y^{2}+ \\
\quad+e x y^{5},|c|+|e| \neq 0\end{array}$ & $\begin{array}{c}t^{2} z+g(z, x, y) \subset \mathbb{P}(4,5,3,2) \\
\text { Diff }=\frac{1}{2} \Delta_{3}\end{array}$ & 2 \\
\hline 113 & $\begin{array}{c}z^{2} x+t x^{3}+x y^{n} \\
n=7 ; 9\end{array}$ & $\begin{array}{c}t^{2} z+z^{2} x+t x^{3}+x y \subset \mathbb{P}(4,5,3,10) \\
\text { Diff }=\left(0,0,0, \frac{n-1}{n}\right)+\frac{4}{5} \Gamma_{3}+\frac{1}{2} \Delta_{3}\end{array}$ & 7,10 \\
\hline 114 & $\begin{array}{l}z^{2} x+t x^{3}+z y^{6}+ \\
+b x^{3} y^{3}\end{array}$ & $\begin{array}{c}t^{2} z+z^{2} x+t x^{3}+z y^{2}+b x^{3} y \subset \mathbb{P}(4,5,3,4) \\
\text { Diff }=\left(0,0,0, \frac{2}{3}\right)+\frac{3}{4} \Delta_{3}\end{array}$ & 4 \\
\hline 115 & $\begin{array}{c}z^{2} x+t x^{3}+z y^{n} \\
n=5 ; 7\end{array}$ & $\begin{array}{c}t^{2} z+z^{2} x+t x^{3}+z y \subset \mathbb{P}(4,5,3,8) \\
\text { Diff }=\left(0,0,0, \frac{n-1}{n}\right)+\frac{3}{4} \Delta_{3}\end{array}$ & 5,8 \\
\hline 116 & $z^{2} x+t x^{i} f_{3-i}\left(x, y^{2}\right)$ & $\begin{array}{c}t^{2} z+z^{2} x+t x^{i} f_{3-i}(x, y) \subset \mathbb{P}(4,5,3,3) \\
\text { Diff }=\left(0,0,0, \frac{1}{2}\right)+\frac{2}{3} \Gamma_{2}\end{array}$ & 3 \\
\hline 117 & $\begin{array}{l}z^{2} x+t x^{3}+t y^{n} \\
n=4 ; 5 ; 7,8\end{array}$ & $\begin{array}{c}t^{2} z+z^{2} x+t x^{3}+t y \subset \mathbb{P}(4,5,3,9) \\
\text { Diff }=\left(0,0,0, \frac{n-1}{n}\right)+\frac{2}{3} \Gamma_{2}\end{array}$ & $4,5,9$ \\
\hline 118 & $z^{2} x+t x^{3}+t x y^{5}$ & $\begin{array}{c}t^{2} z+z^{2} x+t x^{3}+t x y \subset \mathbb{P}(4,5,3,6) \\
\text { Diff }=\left(0,0,0, \frac{4}{5}\right)+\frac{2}{3} \Gamma_{2}+\frac{1}{2} \Delta_{3}\end{array}$ & 6 \\
\hline
\end{tabular}




\begin{tabular}{|c|c|c|c|}
\hline 119 & $z^{2} x+t x^{4}+x y^{5}$ & $\begin{array}{c}t^{2} z+z^{2} x+t x^{4}+x y \subset \mathbb{P}(5,7,3,14) \\
\text { Diff }=\left(0,0,0, \frac{4}{5}\right)+\frac{6}{7} \Gamma_{3}\end{array}$ & 7 \\
\hline 120 & $\begin{array}{c}z^{2} x+t x^{4}+a z x y^{3}+ \\
+b x y^{6}\end{array}$ & $\begin{array}{c}t^{2} z+z^{2} x+t x^{4}+a z x y+b x y^{2} \subset \mathbb{P}(5,7,3,7) \\
\text { Diff }=\left(0,0,0, \frac{2}{3}\right)+\frac{6}{7} \Gamma_{3}\end{array}$ & 7 \\
\hline 121 & $\begin{array}{c}z^{2} x+t x^{4}+z y^{4}+ \\
+b x^{4} y^{2}\end{array}$ & $\begin{array}{c}t^{2} z+z^{2} x+t x^{4}+z y^{2}+b x^{4} y \subset \mathbb{P}(5,7,3,5) \\
\text { Diff }=\left(0,0,0, \frac{1}{2}\right)+\frac{4}{5} \Delta_{3}\end{array}$ & 5 \\
\hline 122 & $\begin{array}{c}z^{2} x+t x^{i} y^{j} f_{4-i-j}(x, y) \\
i=0 \& j=1\end{array}$ & $\begin{array}{c}t^{2} z+g(z, x, y) \subset \mathbb{P}(5,7,3,3) \\
\text { Diff }=\frac{2}{3} \Gamma_{2}\end{array}$ & 3 \\
\hline 123 & $z^{2} x+t x^{4}+t y^{5}$ & $\begin{array}{c}t^{2} z+z^{2} x+t x^{4}+t y \subset \mathbb{P}(5,7,3,12) \\
\text { Diff }=\left(0,0,0, \frac{4}{5}\right)+\frac{2}{3} \Gamma_{2}\end{array}$ & 6 \\
\hline 124 & $z^{2} x+t x^{4}+t x y^{4}$ & $\begin{array}{c}t^{2} z+z^{2} x+t x^{4}+t x y \subset \mathbb{P}(5,7,3,9) \\
\text { Diff }=\left(0,0,0, \frac{3}{4}\right)+\frac{2}{3} \Gamma_{2}\end{array}$ & 9 \\
\hline 125 & $\begin{array}{c}z^{2} x+t x^{3} y+x y^{n} \\
n=5 ; 7\end{array}$ & $\begin{array}{c}t^{2} z+g(z, x, y) \subset \mathbb{P}(4 n-2,5 n, 3 n-4,10) \\
\text { Diff }=\frac{4}{5} \Gamma_{3}+\frac{1}{2} \Delta_{3}\end{array}$ & 6,10 \\
\hline 126 & $\begin{array}{l}z^{2} x+t x^{3} y+a z x y^{3}+ \\
+x y^{6}\end{array}$ & $\begin{array}{c}t^{2} z+g(z, x, y) \subset \mathbb{P}(11,15,7,5) \\
\text { Diff }=\frac{4}{5} \Gamma_{3}\end{array}$ & 5 \\
\hline 127 & $\begin{array}{c}z^{2} x+t x^{3} y+z y^{4}+ \\
+b x^{3} y^{3}\end{array}$ & $\begin{array}{c}t^{2} z+g(z, x, y) \subset \mathbb{P}(8,11,5,4) \\
\text { Diff }=\frac{3}{4} \Delta_{3}\end{array}$ & 4 \\
\hline 128 & $z^{2} x+t x^{3} y+z y^{5}$ & $\begin{array}{c}t^{2} z+z^{2} x+t x^{3} y+z y^{5} \subset \mathbb{P}(20,27,13,8) \\
\text { Diff }=\frac{3}{4} \Delta_{3}\end{array}$ & 8 \\
\hline 129 & $\begin{array}{l}z^{2} x+t x^{3} y+t y^{n} \\
n=5 ; 6\end{array}$ & $\begin{aligned} t^{2} z+g(z, x, y) \subset & \mathbb{P}(4 n-1,5 n+1,3 n-3,9) \\
& \text { Diff }=\frac{2}{3} \Gamma_{2}\end{aligned}$ & 6,9 \\
\hline 130 & $z^{2} x+t x^{3} y+t x y^{4}$ & $\begin{array}{c}t^{2} z+g(z, x, y) \subset \mathbb{P}(14,19,9,6) \\
\text { Diff }=\frac{2}{3} \Gamma_{2}+\frac{1}{2} \Delta_{3}\end{array}$ & 6 \\
\hline 131 & $\begin{array}{l}z^{2} y+x^{5} y+a t y^{5}+ \\
\quad+b z y^{4}+c y^{7}\end{array}$ & $\begin{array}{c}t^{2} z+g\left(z, x^{1 / 5}, y\right) \subset \mathbb{P}(2,3,6,1) \\
\text { Diff }=\left(0,0, \frac{4}{5}, 0\right)+\frac{2}{3} \Gamma_{4}+\frac{1}{2} \Delta_{4}\end{array}$ & 5 \\
\hline 132 & $\begin{array}{c}z^{2} y+x^{5} y+y^{n} \\
n=8 ; 10\end{array}$ & $\begin{array}{c}t z+z^{2} y+x y+y^{n} \subset \mathbb{P}(n+1, n-1,2 n-2,2) \\
\text { Diff }=\left(0,0, \frac{4}{5}, 0\right)+\frac{1}{2} \Gamma+\frac{2 n-3}{2 n-2} \Gamma_{4}\end{array}$ & 15,20 \\
\hline 133 & $\begin{array}{c}z^{2} y+x^{5} y+a z y^{5}+ \\
+b y^{9}\end{array}$ & $\begin{array}{c}t z+z^{2} y+x y+a z y^{5}+b y^{9} \subset \mathbb{P}(5,4,8,1) \\
\text { Diff }=\left(0,0, \frac{4}{5}, 0\right)+\frac{1}{2} \Gamma+\frac{7}{8} \Gamma_{4}\end{array}$ & 10 \\
\hline 134 & $\begin{array}{c}z^{2} y+x^{5} y+x y^{n} \\
n=6 ; 8\end{array}$ & $\begin{array}{c}t z+g(z, x, y) \subset \mathbb{P}(5 n+3,5 n-5,2 n-2,8) \\
\text { Diff }=\frac{1}{2} \Gamma+\frac{2 n-3}{2 n-2} \Gamma_{4}\end{array}$ & $\overline{11,16}$ \\
\hline 135 & $\begin{array}{c}z^{2} y+x^{5} y+a x^{3} y^{4}+ \\
+b x y^{7}\end{array}$ & $\begin{array}{c}t z+g(z, x, y) \subset \mathbb{P}(19,15,6,4) \\
\text { Diff }=\frac{1}{2} \Gamma+\frac{5}{6} \Gamma_{4}\end{array}$ & 8 \\
\hline 136 & $\begin{aligned} z^{2} y+ & x^{5} y+a z x y^{3}+ \\
& +b x^{2} y^{5}\end{aligned}$ & $\begin{array}{c}t z+g(z, x, y) \subset \mathbb{P}(13,10,4,3) \\
\text { Diff }=\frac{1}{2} \Gamma+\frac{3}{4} \Gamma_{4}\end{array}$ & 6 \\
\hline 137 & $z^{2} y+x^{5} y+x^{2} y^{6}$ & $\begin{array}{c}t z+z^{2} y+x^{5} y+x^{2} y^{6} \subset \mathbb{P}(31,25,10,6) \\
\text { Diff }=\frac{1}{2} \Gamma+\frac{9}{10} \Gamma_{4}\end{array}$ & 12 \\
\hline 138 & $\begin{array}{l}z^{2} y+x^{5} y+t y^{n} \\
n=6 ; 7\end{array}$ & $\begin{array}{c}t^{2} z+z^{2} y+x y+t y^{n} \subset \mathbb{P}(n+1,2 n-1,4 n-2,3) \\
\text { Diff }=\left(0,0, \frac{4}{5}, 0\right)+\frac{2 n-2}{2 n-1} \Gamma_{4}+\frac{n-6}{n-5} \Delta_{4}\end{array}$ & 11,15 \\
\hline 139 & $\begin{array}{c}z^{2} y+x^{5} y+t x y^{n} \\
n=4 ; 5\end{array}$ & $\begin{array}{c}t^{2} z+g(z, x, y) \subset \mathbb{P}(5 n+3,10 n-5,4 n-2,11) \\
\text { Diff }=\frac{2 n-2}{2 n-1} \Gamma_{4}+\frac{n-4}{n-3} \Delta_{4}\end{array}$ & 7,11 \\
\hline 140 & $z^{2} y+x^{5} y+t x^{2} y^{3}$ & $\begin{array}{c}t^{2} z+z^{2} y+x^{5} y+t x^{2} y^{3} \subset \mathbb{P}(16,25,10,7) \\
\text { Diff }=\frac{4}{5} \Gamma_{4}+\frac{1}{2} \Delta_{4}\end{array}$ & 7 \\
\hline
\end{tabular}




\begin{tabular}{|c|c|c|c|}
\hline 141 & $z^{2} y+z x^{3}+y^{10}$ & $\begin{array}{c}t z+z^{2} y+z x+y^{10} \subset \mathbb{P}(11,9,11,2) \\
\text { Diff }=\left(\frac{1}{2}, 0, \frac{2}{3}, 0\right)+\frac{10}{11} \Delta_{4}\end{array}$ & 12 \\
\hline 142 & $\begin{array}{c}z^{2} y+z x^{3}+a t y^{5}+ \\
+b z y^{4}+c x^{3} y^{3}+d y^{7}+ \\
e t x^{3} y,|a|+|b|+|d| \neq 0\end{array}$ & $\begin{array}{c}t^{2} z+g\left(z, x^{1 / 3}, y\right) \subset \mathbb{P}(2,3,4,1) \\
\text { Diff }=\left(0,0, \frac{2}{3}, 0\right)+\frac{1}{2} \Delta_{4}\end{array}$ & 3 \\
\hline 143 & $\begin{array}{c}z^{2} y+z x^{3}+a z x y^{3}+ \\
+b x^{4} y^{2}+c x^{2} y^{5}+d y^{8}\end{array}$ & $\begin{array}{c}t z+g(z, x, y) \subset \mathbb{P}(9,7,3,2) \\
\text { Diff }=\left(\frac{1}{2}, 0,0,0\right)+\frac{2}{3} \Delta_{4}\end{array}$ & 4 \\
\hline 144 & $\begin{array}{c}z^{2} y+z x^{3}+a z y^{5}+ \\
+b x^{3} y^{4}+c y^{9}\end{array}$ & $\begin{array}{c}t z+z^{2} y+z x+a z y^{5}+b x y^{4}+c y^{9} \subset \mathbb{P}(5,4,5,1) \\
\text { Diff }=\left(\frac{1}{2}, 0, \frac{2}{3}, 0\right)+\frac{4}{5} \Delta_{4}\end{array}$ & 6 \\
\hline 145 & $\begin{array}{c}z^{2} y+z x^{3}+a t x^{2} y^{n}+ \\
+b x y^{2 n+1}, n=2 b \neq 0 ; 3\end{array}$ & $\begin{array}{c}t^{2} z+g(z, x, y) \subset \mathbb{P}(3 n+3,6 n+1,2 n+2,5) \\
\text { Diff }=\frac{n}{n+1} \Delta_{4}\end{array}$ & 3,5 \\
\hline 146 & $\begin{array}{c}z^{2} y+z x^{3}+x y^{n} \\
n=6 ; 8\end{array}$ & $\begin{array}{c}t z+g(z, x, y) \subset \mathbb{P}(3 n+3,3 n-2, n+1,5) \\
\text { Diff }=\left(\frac{1}{2}, 0,0,0\right)+\frac{n}{n+1} \Delta_{4}\end{array}$ & 7,10 \\
\hline 147 & $\begin{array}{c}z^{2} y+z x^{3}+x^{2} y^{n} \\
n=4 ; 6\end{array}$ & $\begin{array}{c}t z+g(z, x, y) \subset \mathbb{P}(3 n+3,3 n-1, n+1,4) \\
\text { Diff }=\left(\frac{1}{2}, 0,0,0\right)+\frac{n}{n+1} \Delta_{4}\end{array}$ & 5,8 \\
\hline 148 & $\begin{array}{l}z^{2} y+z x^{3}+t y^{n} \\
\quad n=4 ; 6 ; 7\end{array}$ & $\begin{array}{c}t^{2} z+g\left(z, x^{1 / 3}, y\right) \subset \mathbb{P}(n+1,2 n-1,2 n+2,3) \\
\text { Diff }=\left(0,0, \frac{2}{3}, 0\right)+\frac{n}{n+1} \Delta_{4}\end{array}$ & $5,7,9$ \\
\hline 149 & $\begin{array}{c}z^{2} y+z x^{3}+t x y^{n} \\
n=3 ; 4 ; 5\end{array}$ & $\begin{array}{c}t^{2} z+g(z, x, y) \subset \mathbb{P}(3 n+3,6 n-1,2 n+2,7) \\
\operatorname{Diff}=\frac{n}{n+1} \Delta_{4}\end{array}$ & $4,5,7$ \\
\hline 150 & $\begin{array}{c}z^{2} y+t x^{4}+a t x^{2} y^{3}+ \\
+b t y^{6}\end{array}$ & $\begin{array}{c}t^{2} z+z^{2} y+t x^{2}+a t x y^{3}+b t y^{6} \subset \mathbb{P}(7,11,9,3) \\
\text { Diff }=\left(0,0, \frac{1}{2}, 0\right)+\frac{2}{3} \Gamma_{2}\end{array}$ & 6 \\
\hline 151 & $z^{2} y+t x^{4}+t y^{7}$ & $\begin{array}{c}t^{2} z+z^{2} y+t x+t y^{7} \subset \mathbb{P}(8,13,21,3) \\
\text { Diff }=\left(0,0, \frac{3}{4}, 0\right)+\frac{2}{3} \Gamma_{2}\end{array}$ & 12 \\
\hline 152 & $\begin{array}{c}z^{2} y+t x^{4}+t x y^{n} \\
n=4 ; 5\end{array}$ & $\begin{aligned} t^{2} z+g(z, x, y) & \subset \mathbb{P}(4 n+3,8 n-3,3 n, 9) \\
& \operatorname{Diff}=\frac{2}{3} \Gamma_{2}\end{aligned}$ & 6,9 \\
\hline
\end{tabular}

8. Singularity $-t^{2} x+g(z, x, y)$.

\begin{tabular}{|c|c|c|c|}
\hline 1 & $z^{4}+x^{3} y+x y^{6}$ & $\begin{array}{c}t^{2} x+z+x^{3} y+x y^{6} \subset \mathbb{P}(6,17,5,2) \\
\mathbb{P}(3,5,1), \text { Diff }=\frac{3}{\Delta} \Delta+\frac{7}{9} \Delta_{3}\end{array}$ & 8 \\
\hline 2 & $z^{4}+x^{5}+x y^{5}$ & $\begin{array}{c}t^{2} x+z+x^{5}+x y \subset \mathbb{P}(2,5,1,4) \\
\mathbb{P}(1,1,2), \text { Diff }=\left(0,0,0, \frac{4}{5}\right)+\frac{3}{4} \Delta+\frac{7}{8} \Delta_{3}\end{array}$ & 16 \\
\hline 3 & $z^{4}+x^{4} y+x y^{5}$ & $\begin{array}{c}t^{2} x+z^{2}+x^{4} y+x y^{5} \subset \mathbb{P}(15,19,8,6) \\
\text { Diff }=\frac{1}{2} \Delta+\frac{5}{6} \Delta_{3}\end{array}$ & $\overline{12}$ \\
\hline 4 & $\begin{array}{c}z^{4}+x^{4} y+z y^{n} \\
n=4 ; 5\end{array}$ & $\begin{array}{c}t x+g(z, x, y) \subset \mathbb{P}(12 n+3,4 n, 4 n-3,12) \\
\text { Diff }=\left(\frac{1}{2}, 0,0,0\right)+\frac{2}{3} \Delta_{3}\end{array}$ & 10,24 \\
\hline 5 & $z^{4}+x^{4} y+t z y^{2}$ & $\begin{array}{c}t^{2} x+z^{4}+x^{4} y+t z y^{2} \subset \mathbb{P}(27,17,14,12) \\
\text { Diff }=\frac{2}{3} \Delta_{3}\end{array}$ & 6 \\
\hline 6 & $z^{4}+z x^{2} y+x y^{6}$ & $\begin{array}{c}t^{2} x+z^{4}+z x^{2} y+x y^{6} \subset \mathbb{P}(15,11,14,5) \\
\text { Diff }=\frac{4}{5} \Delta_{3}\end{array}$ & 5 \\
\hline 7 & $\begin{array}{c}z^{4}+z x^{2} y+x y^{n} \\
n=5 ; 7\end{array}$ & $\begin{array}{c}t x+g(z, x, y) \subset \mathbb{P}(5 n, 2 n-1,3 n-4,5) \\
\text { Diff }=\left(\frac{1}{2}, 0,0,0\right)+\frac{4}{5} \Delta_{3}\end{array}$ & 6,10 \\
\hline 8 & $\begin{array}{c}z^{4}+z x^{2} y+z y^{n} \\
n=4 ; 8\end{array}$ & $\begin{array}{c}t x+g(z, x, y) \subset \mathbb{P}(5 n+3,2 n, 3 n-3,6) \\
\text { Diff }=\frac{1}{2} \Gamma+\frac{5}{6} \Gamma_{2}\end{array}$ & 6,12 \\
\hline 9 & $\begin{array}{c}z^{4}+z y x^{i} f_{2-i}\left(x, y^{2}\right)+ \\
a t z^{2} y, i=0 \|(a \neq 0 \& i \leq 1)\end{array}$ & $\begin{array}{c}t^{2} x+g(z, x, y) \subset \mathbb{P}(7,5,6,3) \\
\text { Diff }=\frac{2}{3} \Gamma_{2}\end{array}$ & 3 \\
\hline 10 & $z^{4}+z x^{2} y+a z x y^{4}+$ & $t x+g(z, x, y) \subset \mathbb{P}(19,7,9,3)$ & 6 \\
\hline
\end{tabular}




\begin{tabular}{|c|c|c|c|}
\hline & $+b z y^{7}$ & Diff $=\frac{1}{2} \Gamma+\frac{5}{6} \Gamma_{2}$ & \\
\hline 11 & $z^{4}+z x^{4}+x y^{5}$ & $\begin{array}{c}t x+z^{4}+z x^{4}+x y \subset \mathbb{P}(13,4,3,13) \\
\text { Diff }=\left(\frac{1}{2}, 0,0, \frac{4}{5}\right)+\frac{12}{13} \Delta_{3}\end{array}$ & 26 \\
\hline 12 & $\begin{array}{c}z^{4}+z x^{i} y^{j} f_{4-i-j}(x, y) \\
i=0 \& j \leq 2\end{array}$ & $\begin{array}{c}t x+g(z, x, y) \subset \mathbb{P}(13,4,3,3) \\
\text { Diff }=\frac{1}{2} \Gamma+\frac{5}{6} \Gamma_{2}\end{array}$ & 6 \\
\hline 13 & $z^{4}+z x^{3} y+x y^{5}$ & $\begin{array}{c}t x+g(z, x, y) \subset \mathbb{P}(45,14,11,9) \\
\text { Diff }=\left(\frac{1}{2}, 0,0,0\right)+\frac{8}{9} \Delta_{3}\end{array}$ & 18 \\
\hline 14 & $z^{4}+z x^{3} y+t z y^{2}$ & $\begin{array}{c}t^{2} x+z^{4}+z x^{3} y+t z y^{2} \subset \mathbb{P}(21,13,10,9) \\
\text { Diff }=\frac{2}{3} \Delta_{3}\end{array}$ & 9 \\
\hline 15 & $z^{4}+z x^{2} y^{2}+x y^{5}$ & $\begin{array}{c}t x+g(z, x, y) \subset \mathbb{P}(25,8,7,5) \\
\text { Diff }=\left(\frac{1}{2}, 0,0,0\right)+\frac{4}{5} \Delta_{3}\end{array}$ & 10 \\
\hline 16 & $z^{4}+z x^{2} y^{2}+z y^{5}$ & $\begin{array}{c}t x+z^{4}+z x^{2} y^{2}+z y^{5} \subset \mathbb{P}(31,10,9,6) \\
\text { Diff }=\frac{1}{2} \Gamma+\frac{5}{6} \Gamma_{2}\end{array}$ & 12 \\
\hline 17 & $z^{3} x+x^{2} y^{2}+y^{9}$ & $\begin{array}{c}t x+z x+x^{2} y^{2}+y^{9} \subset \mathbb{P}(11,11,7,2) \\
\text { Diff }=\left(\frac{1}{2}, \frac{2}{3}, 0,0\right)+\frac{10}{11} \Upsilon_{4}\end{array}$ & 12 \\
\hline 18 & $\begin{array}{c}z^{3} x+x^{2} y^{2}+a z^{4} y+ \\
+b z^{2} y^{4}+c y^{7}+d z x y^{3}\end{array}$ & $\begin{array}{c}t x+g(z, x, y) \subset \mathbb{P}(9,3,5,2) \\
\text { Diff }=\left(\frac{1}{2}, 0,0,0\right)+\frac{2}{3} \Upsilon_{4}\end{array}$ & 4 \\
\hline 19 & $\begin{aligned} z^{3} x & +x^{2} y^{2}+a z^{3} y^{3}+ \\
& +b x y^{5}+c y^{8}\end{aligned}$ & $\begin{array}{c}t x+g\left(z^{1 / 3}, x, y\right) \subset \mathbb{P}(5,5,3,1) \\
\text { Diff }=\left(\frac{1}{2}, \frac{2}{3}, 0,0\right)+\frac{4}{5} \Upsilon_{4}\end{array}$ & 6 \\
\hline 20 & $\begin{array}{l}z^{3} x+x^{2} y^{2}+a t z^{2} y+ \\
\quad+b z y^{4}, b \neq 0\end{array}$ & $\begin{array}{c}t^{2} x+g(z, x, y) \subset \mathbb{P}(9,6,8,5) \\
\text { Diff }=\frac{2}{3} \Gamma_{2}\end{array}$ & 3 \\
\hline 21 & $\begin{array}{c}z^{3} x+x^{2} y^{2}+z y^{n} \\
n=5 ; 7\end{array}$ & $\begin{array}{c}t x+g(z, x, y) \subset \mathbb{P}(3 n+6, n+2,3 n-4,5) \\
\text { Diff }=\left(\frac{1}{2}, 0,0,0\right)+\frac{n+1}{n+2} \Upsilon_{4}\end{array}$ & 7,10 \\
\hline 22 & $\begin{array}{c}z^{3} x+x^{2} y^{2}+a t z^{2} y^{2}+ \\
+b z y^{6}\end{array}$ & $\begin{array}{c}t^{2} x+g\left(z, x, y^{1 / 2}\right) \subset \mathbb{P}(6,4,7,5) \\
\text { Diff }=\frac{1}{2} \Omega+\frac{3}{4} \Upsilon_{4}\end{array}$ & 5 \\
\hline 23 & $\begin{array}{c}z^{3} x+x^{2} y^{2}+z^{2} y^{n} \\
n=3 ; 5\end{array}$ & $\begin{array}{c}t x+g(z, x, y) \subset \mathbb{P}(3 n+6, n+2,3 n-2,4) \\
\text { Diff }=\left(\frac{1}{2}, 0,0,0\right)+\frac{n+1}{n+2} \Upsilon_{4}\end{array}$ & 5,8 \\
\hline 24 & $z^{3} x+x^{2} y^{2}+t y^{5}$ & $\begin{array}{c}t^{2} x+z x+x^{2} y^{2}+t y^{5} \subset \mathbb{P}(7,14,8,3) \\
\text { Diff }=\left(0, \frac{2}{3}, 0,0\right)+\frac{6}{7} \Upsilon_{4}\end{array}$ & 7 \\
\hline 25 & $z^{3} x+x^{2} y^{2}+t y^{6}$ & $\begin{array}{c}t^{2} x+z x+x^{2} y+t y^{3} \subset \mathbb{P}(4,8,5,3) \\
\text { Diff }=\left(0, \frac{2}{3}, 0,0\right)+\frac{1}{2} \Omega+\frac{7}{8} \Upsilon_{4}\end{array}$ & 9 \\
\hline 26 & $\begin{array}{c}z^{3} x+x^{2} y^{2}+t z y^{2 n} \\
n=1 ; 2\end{array}$ & $\begin{array}{c}t^{2} x+g\left(z, x, y^{1 / 2}\right) \subset \mathbb{P}(3 n+3,2 n+2,6 n-1,7) \\
\text { Diff }=\frac{1}{2} \Omega+\frac{2 n+1}{2 n+2} \Upsilon_{4}\end{array}$ & 4,7 \\
\hline 27 & $z^{3} x+x^{2} y^{2}+t z y^{3}$ & $\begin{array}{c}t^{2} x+z^{3} x+x^{2} y^{2}+t z y^{3} \subset \mathbb{P}(15,10,16,7) \\
\text { Diff }=\frac{4}{5} \Upsilon_{4}\end{array}$ & 5 \\
\hline 28 & $\begin{array}{c}z^{3} y+x^{i} y^{j}(x+y)^{k} \\
\cdot f_{5-i-j-k}(x, y)+a t y^{3} \\
j \leq 1 \&(a \neq 0 \|(i=0 \& k \leq 3))\end{array}$ & $\begin{array}{c}t^{2} x+g\left(z^{1 / 3}, x, y\right) \subset \mathbb{P}(2,4,1,1) \\
\text { Diff }=\left(0, \frac{2}{3}, 0,0\right)+\frac{1}{2} \Upsilon_{4}\end{array}$ & 3 \\
\hline 29 & $\begin{array}{l}z^{3} x+\underline{x^{5}}+z x^{2} y^{2}+ \\
+a t z y^{2}+c y^{6}, a \neq 0\end{array}$ & $\begin{array}{c}t^{2} x+g\left(z, x, y^{1 / 2}\right) \subset \mathbb{P}(6,4,3,5) \\
\text { Diff }=\frac{1}{2} \Omega+\frac{3}{4} \Upsilon_{4}\end{array}$ & 6 \\
\hline 30 & $z^{3} x+x^{5}+y^{7}$ & $\begin{array}{c}t^{2} x+z x+x^{5}+y \subset \mathbb{P}(2,4,1,5) \\
\mathbb{P}(1,2,1), \text { Diff }=\left(0, \frac{2}{3}, 0,0\right)+\frac{6}{7} \Omega+\frac{13}{14} \Upsilon_{4}\end{array}$ & 15 \\
\hline 31 & $z^{3} x+\frac{x^{5}}{+t y^{4}}+x^{2} y^{4}+$ & $\begin{array}{c}t^{2} x+z x+\underline{x^{5}}+x^{2} y+t y \subset \mathbb{P}(2,4,1,3) \\
\text { Diff }=\left(0, \frac{2}{3}, 0,0\right)+\frac{3}{4} \Omega+\frac{7}{8} \Upsilon_{4}\end{array}$ & 9 \\
\hline
\end{tabular}




\begin{tabular}{|c|c|c|c|}
\hline 32 & $z^{3} x+x^{5}+z y^{5}$ & $\begin{array}{c}t^{2} x+z^{3} x+x^{5}+z y \subset \mathbb{P}(6,4,3,11) \\
\text { Diff }=\frac{4}{5} \Omega+\frac{9}{10} \Upsilon_{4}\end{array}$ & 11 \\
\hline 33 & $z^{3} x+x^{5}+z^{2} y^{3}$ & $\begin{array}{c}t^{2} x+z^{3} x+x^{5}+z^{2} y \subset \mathbb{P}(6,4,3,7) \\
\text { Diff }=\frac{2}{3} \Omega+\frac{5}{6} \Upsilon_{4}\end{array}$ & 7 \\
\hline 34 & $z^{3} x+\frac{x^{4} y}{+y^{7}}+x^{2} y^{4}+$ & $\begin{array}{c}t x+z x+\frac{x^{4} y}{\overline{\text { Diff }}}=\frac{x^{2} y^{4}+y^{7} \subset \mathbb{P}\left(1, \frac{2}{3}, 0,0\right)+\frac{10}{11} \Upsilon_{4}}{} \\
\end{array}$ & 12 \\
\hline 35 & $\begin{array}{l}z^{3} x+x^{4} y+z x^{2} y^{2}+ \\
\quad+a z^{2} y^{3}+\frac{c x y^{5}}{}\end{array}$ & $\begin{array}{c}t x+g(z, x, y) \subset \mathbb{P}(15,5,4,3) \\
\operatorname{Diff}=\left(\frac{1}{2}, 0,0,0\right)+\frac{2}{3} \Delta_{3}+\frac{4}{5} \Upsilon_{4}\end{array}$ & 6 \\
\hline 36 & $z^{3} x+x^{4} y+z y^{5}$ & $\begin{array}{c}t^{2} x+z^{3} x+x^{4} y+z y^{5} \subset \mathbb{P}(24,16,13,9) \\
\text { Diff }=\frac{2}{3} \Delta_{3}+\frac{7}{8} \Upsilon_{4}\end{array}$ & 9 \\
\hline 37 & $z^{3} x+x^{4} y+t y^{4}$ & $\begin{array}{c}t^{2} x+z x+x^{4} y+t y^{4} \subset \mathbb{P}(13,26,7,5) \\
\text { Diff }=\left(0, \frac{2}{3}, 0,0\right)+\frac{12}{13} \Upsilon_{4}\end{array}$ & 13 \\
\hline 38 & $z^{3} x+x^{4} y+t z y^{2}$ & $\begin{array}{c}t^{2} x+z^{3} x+x^{4} y+t z y^{2} \subset \mathbb{P}(21,14,11,9) \\
\text { Diff }=\frac{2}{3} \Delta_{3}+\frac{6}{7} \Upsilon_{4}\end{array}$ & 7 \\
\hline 39 & $z^{3} x+x^{3} y^{2}+y^{7}$ & $\begin{array}{c}t^{2} x+z x+x^{3} y^{2}+y^{7} \subset \mathbb{P}(8,16,5,3) \\
\text { Diff }=\left(0, \frac{2}{3}, 0,0\right)+\frac{7}{8} \Upsilon_{4}\end{array}$ & 9 \\
\hline 40 & $\begin{aligned} z^{3} x+ & x^{3} y^{2}+t y^{4}+ \\
& +b x y^{5}\end{aligned}$ & $\begin{array}{c}t^{2} x+g\left(z^{1 / 3}, x, y\right) \subset \mathbb{P}(5,10,3,2) \\
\text { Diff }=\left(0, \frac{2}{3}, 0,0\right)+\frac{1}{2} \Gamma_{3}+\frac{4}{5} \Upsilon_{4}\end{array}$ & 6 \\
\hline 41 & $z^{3} x+x^{3} y^{2}+z y^{5}$ & $\begin{array}{c}t^{2} x+z^{3} x+x^{3} y^{2}+z y^{5} \subset \mathbb{P}(18,12,11,7) \\
\text { Diff }=\frac{5}{6} \Upsilon_{4}\end{array}$ & 7 \\
\hline 42 & $\begin{aligned} z^{3} x+ & x^{3} y^{2}+t z y^{2}+ \\
& +b z x y^{3}\end{aligned}$ & $\begin{array}{c}t^{2} x+g(z, x, y) \subset \mathbb{P}(9,6,5,4) \\
\operatorname{Diff}=\frac{1}{2} \Gamma_{3}+\frac{2}{3} \Upsilon_{4}\end{array}$ & 5 \\
\hline 43 & $z^{3} x+x^{3} y^{2}+z^{2} y^{3}$ & $\begin{array}{c}t^{2} x+z^{3} x+x^{3} y^{2}+z^{2} y^{3} \subset \mathbb{P}(12,8,7,5) \\
\text { Diff }=\frac{3}{4} \Upsilon_{4}\end{array}$ & 5 \\
\hline 44 & $z^{3} x+x^{2} y^{3}+y^{8}$ & $\begin{array}{c}t x+z x+x^{2} y^{3}+y^{8} \subset \mathbb{P}(11,11,5,2) \\
\text { Diff }=\left(\frac{1}{2}, \frac{2}{3}, 0,0\right)+\frac{10}{11} \Upsilon_{4}\end{array}$ & 12 \\
\hline 45 & $z^{3} x+x^{2} y^{3}+z y^{6}$ & $\begin{array}{c}t x+z^{3} x+x^{2} y+z y^{2} \subset \mathbb{P}(9,3,4,5) \\
\text { Diff }=\left(\frac{1}{2}, 0,0,0\right)+\frac{2}{3} \Omega+\frac{8}{9} \Upsilon_{4}\end{array}$ & 10 \\
\hline 46 & $\begin{array}{l}z^{3} x+x^{2} y^{3}+a t z^{2} y+ \\
+b z y^{5}\end{array}$ & $\begin{array}{c}t^{2} x+g(z, x, y) \subset \mathbb{P}(12,8,9,5) \\
\text { Diff }=\frac{3}{4} \Upsilon_{4}\end{array}$ & 5 \\
\hline 47 & $z^{3} x+x^{2} y^{3}+z^{2} y^{4}$ & $\begin{array}{c}t x+z^{3} x+x^{2} y^{3}+z^{2} y^{4} \subset \mathbb{P}(21,7,9,4) \\
\text { Diff }=\left(\frac{1}{2}, 0,0,0\right)+\frac{6}{7} \Upsilon_{4} \\
\end{array}$ & 8 \\
\hline 48 & $\begin{array}{c}z^{3} x+x^{2} y^{3}+t y^{n} \\
n=4 ; 5\end{array}$ & $\begin{array}{c}t^{2} x+g\left(z^{1 / 3}, x, y\right) \subset \mathbb{P}(n+3,2 n+6,2 n-3,3) \\
\text { Diff }=\left(0, \frac{2}{3}, 0,0\right)+\frac{n+2}{n+3} \Upsilon_{4}\end{array}$ & 7,9 \\
\hline 49 & $z^{3} x+x^{2} y^{3}+t z y^{2}$ & $\begin{array}{c}t^{2} x+z^{3} x+x^{2} y^{3}+t z y^{2} \subset \mathbb{P}(15,10,9,7) \\
\text { Diff }=\frac{4}{5} \Upsilon_{4}\end{array}$ & 5 \\
\hline 50 & $z^{3} x+x^{2} y^{3}+t z y^{3}$ & $\begin{array}{c}t^{2} x+z^{3} x+x^{2} y+t z y \subset \mathbb{P}(6,4,5,7) \\
\text { Diff }=\frac{2}{3} \Omega+\frac{5}{6} \Upsilon_{4}\end{array}$ & 7 \\
\hline 51 & $z^{3} x+x^{6}+y^{7}$ & $\begin{array}{c}t x+z x+x^{6}+y \subset \mathbb{P}(5,5,1,6) \\
\mathbb{P}^{2}, \text { Diff }=\left(\frac{1}{2}, \frac{2}{3}, 0,0\right)+\frac{6}{7} \Omega+\frac{34}{35} \Upsilon_{4}\end{array}$ & 36 \\
\hline 52 & $\begin{array}{c}z^{3} y+x^{i} y^{j}(x+y)^{k} f_{6-i-j-k} \\
(x, y), \quad k \leq 4 \& i \leq 1 \& j \leq 2\end{array}$ & $\begin{array}{c}t x+g\left(z^{1 / 3}, x, y\right) \subset \mathbb{P}(5,5,1,1) \\
\text { Diff }=\left(\frac{1}{2}, \frac{2}{3}, 0,0\right)+\frac{4}{5} \Upsilon_{4}\end{array}$ & 6 \\
\hline 53 & $z^{3} x+x^{6}+z y^{5}$ & $\begin{array}{c}t x+z^{3} x+x^{6}+z y \subset \mathbb{P}(15,5,3,13) \\
\text { Diff }=\left(\frac{1}{2}, 0,0,0\right)+\frac{4}{5} \Omega+\frac{24}{25} \Upsilon_{4}\end{array}$ & 26 \\
\hline
\end{tabular}




\begin{tabular}{|c|c|c|c|}
\hline$\overline{54}$ & $z^{3} x+x^{6}+z^{2} y^{3}$ & $\begin{array}{c}t x+z^{3} x+x^{6}+z^{2} y \subset \mathbb{P}(15,5,3,8) \\
\text { Diff }=\left(\frac{1}{2}, 0,0,0\right)+\frac{2}{3} \Omega+\frac{14}{15} \Upsilon_{4}\end{array}$ & 16 \\
\hline$\overline{55}$ & $z^{3} x+x^{6}+t y^{4}$ & $\begin{array}{c}t^{2} x+z x+x^{6}+t y \subset \mathbb{P}(5,10,2,7) \\
\text { Diff }=\left(0, \frac{2}{3}, 0,0\right)+\frac{3}{4} \Omega+\frac{19}{20} \Upsilon_{4}\end{array}$ & 21 \\
\hline 56 & $z^{3} x+x^{6}+t z y^{2}$ & $\begin{array}{c}t^{2} x+z^{3} x+x^{6}+t z y \subset \mathbb{P}(15,10,6,11) \\
\text { Diff }=\frac{1}{2} \Omega+\frac{9}{10} \Upsilon_{4}\end{array}$ & 11 \\
\hline 57 & $z^{3} x+x^{5} y+y^{7}$ & $\begin{array}{c}t x+z x+x^{5} y+y^{7} \subset \mathbb{P}(29,29,6,5) \\
\text { Diff }=\left(\frac{1}{2}, \frac{2}{3}, 0,0\right)+\frac{28}{29} \Upsilon_{4}\end{array}$ & 30 \\
\hline 58 & $z^{3} x+x^{5} y+z y^{5}$ & $\begin{array}{c}t x+z^{3} x+x^{5} y+z y^{5} \subset \mathbb{P}(63,21,13,11) \\
\text { Diff }=\left(\frac{1}{2}, 0,0,0\right)+\frac{20}{21} \Upsilon_{4}\end{array}$ & 22 \\
\hline$\overline{59}$ & $z^{3} x+x^{5} y+z^{2} y^{3}$ & $\begin{array}{c}t x+z^{3} x+x^{5} y+z^{2} y^{3} \subset \mathbb{P}(39,13,8,7) \\
\text { Diff }=\left(\frac{1}{2}, 0,0,0\right)+\frac{12}{13} \Upsilon_{4}\end{array}$ & 14 \\
\hline 60 & $z^{3} x+x^{5} y+t y^{4}$ & $\begin{array}{c}t^{2} x+z x+x^{5} y+t y^{4} \subset \mathbb{P}(17,34,7,6) \\
\text { Diff }=\left(0, \frac{2}{3}, 0,0\right)+\frac{1}{2} \Gamma_{3}+\frac{16}{17} \Upsilon_{4}\end{array}$ & 18 \\
\hline 61 & $z^{3} x+x^{5} y+t z y^{2}$ & $\begin{array}{c}t^{2} x+z^{3} x+x^{5} y+t z y^{2} \subset \mathbb{P}(27,18,11,10) \\
\text { Diff }=\frac{1}{2} \Gamma_{3}+\frac{8}{9} \Upsilon_{4}\end{array}$ & 10 \\
\hline 62 & $z^{3} x+x^{4} y^{2}+y^{7}$ & $\begin{array}{c}t x+z x+x^{4} y^{2}+y^{7} \subset \mathbb{P}(23,23,5,4) \\
\text { Diff }=\left(\frac{1}{2}, \frac{2}{3}, 0,0\right)+\frac{22}{23} \Upsilon_{4}\end{array}$ & 24 \\
\hline 63 & $z^{3} x+x^{4} y^{2}+z y^{5}$ & $\begin{array}{c}t x+z^{3} x+x^{4} y^{2}+z y^{5} \subset \mathbb{P}(51,17,11,9) \\
\text { Diff }=\left(\frac{1}{2}, 0,0,0\right)+\frac{2}{3} \Delta_{3}+\frac{16}{17} \Upsilon_{4}\end{array}$ & 18 \\
\hline 64 & $z^{3} x+x^{4} y^{2}+z^{2} y^{3}$ & $\begin{array}{c}t x+z^{3} x+x^{4} y^{2}+z^{2} y^{3} \subset \mathbb{P}(33,11,7,6) \\
\text { Diff }=\left(\frac{1}{2}, 0,0,0\right)+\frac{2}{3} \Delta_{3}+\frac{10}{11} \Upsilon_{4}\end{array}$ & $\overline{12}$ \\
\hline 65 & $z^{3} x+x^{4} y^{2}+t y^{4}$ & $\begin{array}{c}t^{2} x+z x+x^{4} y+t y^{2} \subset \mathbb{P}(7,14,3,5) \\
\text { Diff }=\left(0, \frac{2}{3}, 0,0\right)+\frac{1}{2} \Omega+\frac{13}{14} \Upsilon_{4}\end{array}$ & 15 \\
\hline 66 & $z^{3} x+x^{4} y^{2}+t z y^{2}$ & $\begin{array}{c}t^{2} x+z^{3} x+x^{4} y+t z y \subset \mathbb{P}(12,8,5,9) \\
\text { Diff }=\frac{1}{2} \Omega+\frac{2}{3} \Delta_{3}+\frac{7}{8} \Upsilon_{4}\end{array}$ & 9 \\
\hline 67 & $z^{3} x+x^{3} y^{3}+y^{7}$ & $\begin{array}{c}t x+z x+x^{3} y^{3}+y^{7} \subset \mathbb{P}(17,17,4,3) \\
\text { Diff }=\left(\frac{1}{2}, \frac{2}{3}, 0,0\right)+\frac{16}{17} \Upsilon_{4}\end{array}$ & 18 \\
\hline 68 & $z^{3} x+x^{3} y^{3}+z y^{5}$ & $\begin{array}{c}t x+z^{3} x+x^{3} y^{3}+z y^{5} \subset \mathbb{P}(39,13,9,7) \\
\text { Diff }=\left(\frac{1}{2}, 0,0,0\right)+\frac{12}{13} \Upsilon_{4}\end{array}$ & 14 \\
\hline 69 & $z^{3} x+\frac{x^{3} y^{3}+}{+z^{2} y^{3}} \underline{z x^{4}}+$ & $\begin{array}{c}t x+z^{3} x+x^{3} y+\underline{z x^{4}}+z^{2} y \subset \mathbb{P}(9,3,2,5) \\
\text { Diff }=\left(\frac{1}{2}, 0,0,0\right)+\frac{2}{3} \Omega+\frac{8}{9} \Upsilon_{4}\end{array}$ & 10 \\
\hline 70 & $z^{3} x+x^{3} y^{3}+t y^{4}$ & $\begin{array}{c}t^{2} x+z x+x^{3} y^{3}+t y^{4} \subset \mathbb{P}(11,22,5,4) \\
\text { Diff }=\left(0, \frac{2}{3}, 0,0\right)+\frac{1}{2} \Gamma_{3}+\frac{10}{11} \Upsilon_{4}\end{array}$ & 12 \\
\hline 71 & $z^{3} x+x^{3} y^{3}+t z y^{2}$ & $\begin{array}{c}t^{2} x+z^{3} x+x^{3} y^{3}+t z y^{2} \subset \mathbb{P}(21,14,9,8) \\
\text { Diff }=\frac{1}{2} \Gamma_{3}+\frac{6}{7} \Upsilon_{4}\end{array}$ & 8 \\
\hline 72 & $z^{3} x+x^{2} y^{4}+z y^{5}$ & $\begin{array}{c}t x+z^{3} x+x^{2} y^{4}+z y^{5} \subset \mathbb{P}(27,9,7,5) \\
\text { Diff }=\left(\frac{1}{2}, 0,0,0\right)+\frac{8}{9} \Upsilon_{4}\end{array}$ & 10 \\
\hline 73 & $z^{3} x+\frac{x^{2} y^{4}}{+z^{2} y^{3}}+z x^{3} y+$ & $\begin{array}{c}t x+g(z, x, y) \subset \mathbb{P}(21,7,5,4) \\
\text { Diff }=\left(\frac{1}{2}, 0,0,0\right)+\frac{6}{7} \Upsilon_{4}\end{array}$ & 8 \\
\hline 74 & $z^{3} x+\frac{x^{2} y^{4}}{+t z y^{2}}+\underline{z x^{4}}+$ & $\begin{array}{c}t^{2} x+z^{3} x+\frac{x^{2} y^{2}}{\text { Diff }}=\frac{z x^{4}}{2} \Omega+t z y \subset \mathbb{P}\left(\frac{5}{6} \Upsilon_{4}\right. \\
\end{array}$ & 7 \\
\hline 75 & $\begin{array}{c}z^{3} x+z x^{2} y+a t z y^{3}+ \\
+b y^{7}\end{array}$ & $\begin{array}{c}t^{2} x+g(z, x, y) \subset \mathbb{P}(12,8,11,5) \\
\text { Diff }=\frac{3}{4} \Upsilon_{4}\end{array}$ & 5 \\
\hline
\end{tabular}




\begin{tabular}{|c|c|c|c|}
\hline 76 & $z^{3} x+z x^{2} y+y^{8}$ & $\begin{array}{c}t x+z^{3} x+z x^{2} y+y^{8} \subset \mathbb{P}(27,9,13,5) \\
\text { Diff }=\left(\frac{1}{2}, 0,0,0\right)+\frac{8}{9} \Upsilon_{4}\end{array}$ & 10 \\
\hline 77 & $\begin{array}{l}z^{3} x+z x^{2} y+a t z^{2} y+ \\
+b z^{2} y^{3}+c x y^{4}, c \neq 0 \\
\end{array}$ & $\begin{array}{c}t^{2} x+g(z, x, y) \subset \mathbb{P}(6,4,5,3) \\
\text { Diff }=\frac{2}{3} \Delta_{3}+\frac{1}{2} \Upsilon_{4}\end{array}$ & 3 \\
\hline 78 & $\begin{array}{c}z^{3} x+z x^{2} y+a z^{2} y^{4}+ \\
+b x y^{5}\end{array}$ & $\begin{array}{l}t x+g(z, x, y) \subset \mathbb{P}(15,5,7,3) \\
\text { Diff }=\left(\frac{1}{2}, 0,0,0\right)+\frac{2}{3} \Delta_{3}+\frac{4}{5} \Upsilon_{4}\end{array}$ & 6 \\
\hline 79 & $z^{3} x+z x^{2} y+z y^{6}$ & $\begin{array}{c}t x+z^{3} x+z x^{2} y+z y^{6} \subset \mathbb{P}(21,7,10,4) \\
\text { Diff }=\frac{1}{2} \Gamma+\frac{3}{4} \Gamma_{2}+\frac{6}{7} \Upsilon_{4}\end{array}$ & 8 \\
\hline 80 & $\begin{array}{l}z^{3} x+z x^{2} y+a z^{3} y^{2}+ \\
\quad+b z x y^{3}+c z y^{5}\end{array}$ & $\begin{array}{c}t x+g(z, x, y) \subset \mathbb{P}(9,3,4,2) \\
\text { Diff }=\frac{1}{2} \Gamma+\frac{3}{4} \Gamma_{2}+\frac{2}{3} \Upsilon_{4}\end{array}$ & 4 \\
\hline 81 & $\begin{array}{c}z^{3} x+z x^{2} y+t y^{n} \\
n=4 ; 5\end{array}$ & $\begin{array}{c}t^{2} x+g(z, x, y) \subset \mathbb{P}(3 n+3,2 n+2,4 n-3,7) \\
\text { Diff }=\frac{n}{n+1} \Upsilon_{4}\end{array}$ & 5,7 \\
\hline 82 & $z^{3} x+z x^{4}+y^{7}$ & $\begin{array}{c}t x+z^{3} x+z x^{4}+y \subset \mathbb{P}(9,3,2,11) \\
\mathbb{P}(3,1,2), \text { Diff }=\left(\frac{1}{2}, 0,0,0\right)+\frac{6}{7} \Omega+\frac{20}{21} \Upsilon_{4}\end{array}$ & 22 \\
\hline 83 & $\begin{array}{c}z^{3} y+z x^{i} y^{j}(x+y)^{k} f_{4-i-j-k} \\
(x, y), \quad k \leq 2 \& i=0 \& j \leq 1\end{array}$ & $\begin{array}{c}t x+g(z, x, y) \subset \mathbb{P}(9,3,2,2) \\
\text { Diff }=\frac{1}{2} \Gamma+\frac{3}{4} \Gamma_{2}+\frac{2}{3} \Upsilon_{4}\end{array}$ & 4 \\
\hline 84 & $z^{3} x+z x^{4}+z y^{5}$ & $\begin{array}{c}t x+z^{3} x+z x^{4}+z y \subset \mathbb{P}(9,3,2,8) \\
\text { Diff }=\frac{1}{2} \Gamma+\frac{3}{4} \Gamma_{2}+\frac{4}{5} \Omega+\frac{14}{15} \Upsilon_{4}\end{array}$ & 16 \\
\hline 85 & $z^{3} x+z x^{4}+t y^{4}$ & $\begin{array}{c}t^{2} x+z^{3} x+z x^{4}+t y \subset \mathbb{P}(9,6,4,13) \\
\text { Diff }=\frac{3}{4} \Omega+\frac{11}{12} \Upsilon_{4}\end{array}$ & 13 \\
\hline 86 & $z^{3} x+z x^{3} y+y^{7}$ & $\begin{array}{c}t x+z^{3} x+z x^{3} y+y^{7} \subset \mathbb{P}(45,15,11,8) \\
\text { Diff }=\left(\frac{1}{2}, 0,0,0\right)+\frac{14}{15} \Upsilon_{4}\end{array}$ & 16 \\
\hline 87 & $\begin{array}{c}z^{3} x+z x^{3} y+t z y^{2}+ \\
+b x y^{5}\end{array}$ & $\begin{array}{c}t^{2} x+g(z, x, y) \subset \mathbb{P}(15,10,7,6) \\
\text { Diff }=\frac{1}{2} \Gamma_{3}+\frac{2}{3} \Delta_{3}+\frac{4}{5} \Upsilon_{4}\end{array}$ & 6 \\
\hline 88 & $\begin{aligned} z^{3} x+ & z x^{3} y+z^{2} y^{3}+ \\
& +a x^{2} y^{4}\end{aligned}$ & $\begin{array}{c}t x+g(z, x, y) \subset \mathbb{P}(21,7,5,4) \\
\text { Diff }=\left(\frac{1}{2}, 0,0,0\right)+\frac{6}{7} \Upsilon_{4}\end{array}$ & 8 \\
\hline 89 & $z^{3} x+z x^{3} y+z y^{5}$ & $\begin{array}{c}t x+g(z, x, y) \subset \mathbb{P}(33,11,8,6) \\
\text { Diff }=\frac{1}{2} \Gamma+\frac{3}{4} \Gamma_{2}+\frac{10}{11} \Upsilon_{4}\end{array}$ & 12 \\
\hline 90 & $z^{3} x+z x^{3} y+t y^{4}$ & $\begin{array}{c}t^{2} x+g(z, x, y) \subset \mathbb{P}(27,18,13,10) \\
\text { Diff }=\frac{1}{2} \Gamma_{3}+\frac{8}{9} \Upsilon_{4}\end{array}$ & 10 \\
\hline 91 & $z^{3} x+z x^{2} y^{2}+y^{7}$ & $\begin{array}{c}t x+z^{3} x+z x^{2} y^{2}+y^{7} \subset \mathbb{P}(27,9,8,5) \\
\text { Diff }=\left(\frac{1}{2}, 0,0,0\right)+\frac{8}{9} \Upsilon_{4}\end{array}$ & 10 \\
\hline 92 & $z^{3} x+z x^{2} y^{2}+z y^{5}$ & $\begin{array}{c}t x+g(z, x, y) \subset \mathbb{P}(21,7,6,4) \\
\text { Diff }=\frac{1}{2} \Gamma+\frac{3}{4} \Gamma_{2}+\frac{6}{7} \Upsilon_{4}\end{array}$ & 8 \\
\hline$\overline{93}$ & $z^{3} x+z x^{2} y^{2}+t y^{4}$ & $\begin{array}{c}t^{2} x+z^{3} x+z x^{2} y+t y^{2} \subset \mathbb{P}(9,6,5,7) \\
\text { Diff }=\frac{1}{2} \Omega+\frac{5}{6} \Upsilon_{4}\end{array}$ & 7 \\
\hline 94 & $\begin{array}{c}z^{3} y+x^{5}+a z y^{5}+ \\
+b y^{7}\end{array}$ & $\begin{array}{c}t^{2} x+g(z, x, y) \subset \mathbb{P}(14,10,7,5) \\
\text { Diff }=\frac{1}{2} \Upsilon_{4}\end{array}$ & 5 \\
\hline 95 & $z^{3} y+x^{5}+y^{9}$ & $\begin{array}{c}t^{2} x+z y+x^{5}+y^{9} \subset \mathbb{P}(18,40,9,5) \\
\text { Diff }=\left(0, \frac{2}{3}, 0,0\right)+\frac{1}{2} \Upsilon_{4}\end{array}$ & 15 \\
\hline 96 & $\begin{array}{l}z^{3} y+x^{5}+a t z y^{2}+ \\
\quad+b z x^{2} y^{2}+c x y^{5}\end{array}$ & $\begin{array}{c}t^{2} x+g(z, x, y) \subset \mathbb{P}(10,7,5,4) \\
\text { Diff }=\frac{1}{2} \Delta_{3}\end{array}$ & 4 \\
\hline 97 & $\begin{array}{c}z^{3} y+x^{5}+a x^{3} y^{3}+ \\
+x y^{6}\end{array}$ & $\begin{array}{c}t^{2} x+z y+x^{5}+a x^{3} y^{3}+x y^{6} \subset \mathbb{P}(6,13,3,2) \\
\text { Diff }=\frac{2}{3} \Delta+\frac{5}{6} \Delta_{3}\end{array}$ & 6 \\
\hline
\end{tabular}




\begin{tabular}{|c|c|c|c|}
\hline 98 & $z^{3} y+x^{5}+x y^{7}$ & $\begin{array}{c}t^{2} x+z y+x^{5}+x y^{7} \subset \mathbb{P}(14,31,7,4) \\
\text { Diff }=\frac{2}{3} \Delta+\frac{5}{6} \Delta_{3}\end{array}$ & 12 \\
\hline 99 & $\begin{aligned} z^{3} y+ & x^{5}+a t y^{5}+ \\
& +b x^{2} y^{5}\end{aligned}$ & $\begin{array}{c}t^{2} x+z y+x^{5}+a t y^{5}+b x^{2} y^{5} \subset \mathbb{P}(10,22,5,3) \\
\text { Diff }=\left(0, \frac{2}{3}, 0,0\right)+\frac{1}{2} \Upsilon_{4}\end{array}$ & 9 \\
\hline 100 & $\begin{array}{c}z^{3} y+x^{5}+z y^{n} \\
n=4 ; 6\end{array}$ & $\begin{aligned} t^{2} x+g(z, x, y) \subset & \mathbb{P}(6 n-2,5 n-5,3 n-1,10) \\
& \text { Diff }=\frac{1}{2} \Delta_{3}\end{aligned}$ & 5,10 \\
\hline 101 & $z^{3} y+x^{5}+z x y^{4}$ & $\begin{array}{c}t^{2} x+z^{3} y+x^{5}+z x y^{4} \subset \mathbb{P}(22,16,11,7) \\
\text { Diff }=\frac{1}{2} \Upsilon_{4}\end{array}$ & 7 \\
\hline 102 & $\begin{array}{l}z^{3} y+x^{4} y+y^{n} \\
n=6 ; 8 ; 12\end{array}$ & $\begin{array}{c}t x+g\left(z^{1 / 3}, x, y\right) \subset \mathbb{P}(3 n+1,4 n-4, n-1,4) \\
\text { Diff }=\left(0, \frac{2}{3}, 0,0\right)+\frac{1}{2} \Gamma+\frac{2 n-3}{2 n-2} \Gamma_{4}\end{array}$ & $10,15,24$ \\
\hline 103 & $z^{3} y+y x^{i} f_{4-i}\left(x, y^{2}\right)$ & $\begin{array}{c}t x+z y+y x^{i} f_{4-i}\left(x, y^{2}\right) \subset \mathbb{P}(7,8,2,1) \\
\text { Diff }=\left(0, \frac{2}{3}, 0,0\right)+\frac{1}{2} \Gamma+\frac{3}{4} \Gamma_{4}\end{array}$ & 6 \\
\hline 104 & $\begin{array}{c}z^{3} y+x^{4} y+a z y^{7}+ \\
+b y^{10}\end{array}$ & $\begin{array}{c}t x+g(z, x, y) \subset \mathbb{P}(31,12,9,4) \\
\text { Diff }=\frac{1}{2} \Gamma+\frac{5}{6} \Gamma_{4}\end{array}$ & 8 \\
\hline 105 & $\begin{array}{c}z^{3} y+x^{4} y+a x^{2} y^{6}+ \\
+b y^{11}\end{array}$ & $\begin{array}{c}t x+g\left(z^{1 / 3}, x, y\right) \subset \mathbb{P}(17,20,5,2) \\
\text { Diff }=\left(0, \frac{2}{3}, 0,0\right)+\frac{1}{2} \Gamma+\frac{9}{10} \Gamma_{4}\end{array}$ & 12 \\
\hline 106 & $\begin{array}{c}z^{3} y+x^{4} y+x y^{n} \\
n=5 ; 9\end{array}$ & $\begin{array}{c}t x+g\left(z^{1 / 3}, x, y\right) \subset \mathbb{P}(3 n, 4 n-4, n-1,3) \\
\text { Diff }=\frac{1}{2} \Gamma+\frac{2}{3} \Delta+\frac{2 n-3}{2 n-2} \Gamma_{4}+\frac{8}{9} \Delta_{3}\end{array}$ & 9,18 \\
\hline 107 & $\begin{array}{c}z^{3} y+x^{4} y+x y^{2 n} \\
n=3,4\end{array}$ & $\begin{array}{c}t^{2} x+g\left(z^{1 / 3}, x, y\right) \subset \mathbb{P}(3 n, 8 n-4,2 n-1,3) \\
\text { Diff }=\frac{2}{3} \Delta+\frac{2 n-2}{2 n-1} \Gamma_{4}+\frac{8}{9} \Delta_{3}\end{array}$ & 9 \\
\hline 108 & $\begin{array}{c}z^{3} y+x^{4} y+z y^{n} \\
n=4 ; 6 ; 8\end{array}$ & $\begin{array}{c}t x+g(z, x, y) \subset \mathbb{P}(9 n-1,4 n-4,3 n-3,8) \\
\text { Diff }=\frac{1}{2} \Gamma+\frac{2 n-3}{2 n-2} \Gamma_{4}\end{array}$ & $7,10,16$ \\
\hline 109 & $z^{3} y+x^{4} y+z x y^{5}$ & $\begin{array}{c}t x+g(z, x, y) \subset \mathbb{P}(41,16,12,5) \\
\text { Diff }=\frac{1}{2} \Gamma+\frac{7}{8} \Gamma_{4}\end{array}$ & 10 \\
\hline 110 & $z^{3} y+x^{4} y+z x y^{4}$ & $\begin{array}{c}t^{2} x+g(z, x, y) \subset \mathbb{P}(16,12,9,5) \\
\text { Diff }=\frac{2}{3} \Gamma_{4}+\frac{3}{4} \Upsilon_{4}\end{array}$ & 5 \\
\hline 111 & $\begin{array}{l}z^{3} y+x^{4} y+t y^{n} \\
n=4 ; 6,7^{*}\end{array}$ & $\begin{array}{c}t^{2} x+g\left(z^{1 / 3}, x, y\right) \subset \mathbb{P}(3 n+1,8 n-4,2 n-1,5) \\
\text { Diff }=\left(0, \frac{2}{3}, 0,0\right)+\frac{2 n-2}{2 n-1} \Gamma_{4}+\left(\frac{1}{2} \Upsilon_{4}\right)^{*}\end{array}$ & 7,15 \\
\hline 112 & $\begin{array}{c}z^{3} y+x^{4} y+t z y^{n} \\
\quad n=2 ; 3\end{array}$ & $\begin{array}{c}t^{2} x+g(z, x, y) \subset \mathbb{P}(9 n-1,8 n-4,6 n-3,7) \\
\text { Diff }=\frac{2 n-2}{2 n-1} \Gamma_{4}+\frac{n-2}{n-1} \Upsilon_{4}\end{array}$ & 6,7 \\
\hline 113 & $z^{3} y+x^{6}+x y^{6}$ & $\begin{array}{c}t^{2} x+z y+x^{6}+x y^{6} \subset \mathbb{P}(15,31,6,5) \\
\text { Diff }=\frac{2}{3} \Delta+\frac{14}{15} \Delta_{3}\end{array}$ & 15 \\
\hline 114 & $\begin{array}{c}z^{3} y+x^{5} y+a z y^{5}+ \\
+b y^{7}\end{array}$ & $\begin{array}{c}t x+g(z, x, y) \subset \mathbb{P}(29,10,6,5) \\
\text { Diff }=\frac{1}{2} \Gamma+\frac{3}{4} \Gamma_{4}\end{array}$ & 10 \\
\hline 115 & $z^{3} y+x^{5} y+y^{8}$ & $\begin{array}{c}t x+z y+x^{5} y+y^{8} \subset \mathbb{P}(33,35,7,5) \\
\text { Diff }=\left(0, \frac{2}{3}, 0,0\right)+\frac{1}{2} \Gamma+\frac{13}{14} \Gamma_{4}\end{array}$ & 30 \\
\hline 116 & $z^{3} y+x^{5} y+x y^{6}$ & $\begin{array}{c}t^{2} x+z y+x^{5} y+x y^{6} \subset \mathbb{P}(12,25,5,4) \\
\text { Diff }=\frac{2}{3} \Delta+\frac{11}{12} \Delta_{3}+\frac{4}{5} \Gamma_{4}\end{array}$ & 12 \\
\hline 117 & $z^{3} y+x^{5} y+x^{2} y^{5}$ & $\begin{array}{c}t x+z y+x^{5} y+x^{2} y^{5} \subset \mathbb{P}(19,20,4,3) \\
\text { Diff }=\left(0, \frac{2}{3}, 0,0\right)+\frac{1}{2} \Gamma+\frac{7}{8} \Gamma_{4}\end{array}$ & 18 \\
\hline 118 & $z^{3} y+x^{5} y+z x y^{4}$ & $\begin{array}{c}t x+z^{3} y+x^{5} y+z x y^{4} \subset \mathbb{P}(43,15,9,7) \\
\text { Diff }=\frac{1}{2} \Gamma+\frac{5}{6} \Gamma_{4}\end{array}$ & 14 \\
\hline 119 & $z^{3} y+x^{5} y+t y^{4}$ & $\begin{array}{c}t^{2} x+z y+x^{5} y+t y^{4} \subset \mathbb{P}(17,35,7,6) \\
\text { Diff }=\left(0, \frac{2}{3}, 0,0\right)+\frac{6}{7} \Gamma_{4}\end{array}$ & 9 \\
\hline
\end{tabular}




\begin{tabular}{|c|c|c|c|}
\hline 120 & $z^{3} y+x^{5} y+t z y^{2}$ & $\begin{array}{c}t^{2} x+z^{3} y+x^{5} y+t z y^{2} \subset \mathbb{P}(22,15,9,8) \\
\text { Diff }=\frac{1}{2} \Delta_{3}+\frac{2}{3} \Gamma_{4}\end{array}$ & 8 \\
\hline 121 & $z^{3} y+x^{4} y^{2}+x y^{6}$ & $\begin{array}{c}t^{2} x+z y+x^{4} y^{2}+x y^{6} \subset \mathbb{P}(9,19,4,3) \\
\text { Diff }=\frac{2}{3} \Delta+\frac{8}{9} \Delta_{3}\end{array}$ & 9 \\
\hline 122 & $z^{3} y+x^{4} y^{2}+x y^{7}$ & $\begin{array}{c}t x+z y+x^{4} y^{2}+x y^{7} \subset \mathbb{P}(21,23,5,3) \\
\text { Diff }=\left(\frac{1}{2}, 0,0,0\right)+\frac{2}{3} \Delta+\frac{8}{9} \Delta_{3}\end{array}$ & 18 \\
\hline 123 & $z^{3} y+x^{4} y^{2}+z y^{6}$ & $\begin{array}{c}t x+z^{3} y+x^{4} y^{2}+z y^{6} \subset \mathbb{P}(55,20,13,8) \\
\text { Diff }=\left(\frac{1}{2}, 0,0,0\right)+\frac{4}{5} \Upsilon_{4}\end{array}$ & 16 \\
\hline 124 & $z^{3} y+z x^{4}+x y^{5}$ & $\begin{array}{c}t^{2} x+z^{3} y+z x^{4}+x y^{5} \subset \mathbb{P}(25,17,11,10) \\
\text { Diff }=\frac{4}{5} \Delta_{3}\end{array}$ & 5 \\
\hline 125 & $z^{3} y+z x^{4}+x y^{6}$ & $\begin{array}{c}t^{2} x+z^{3} y+z x^{4}+x y^{6} \subset \mathbb{P}(30,21,13,10) \\
\text { Diff }=\frac{9}{10} \Delta_{3}+\frac{2}{3} \Upsilon_{4}\end{array}$ & 10 \\
\hline 126 & $z^{3} y+z x^{4}+x^{2} y^{5}$ & $\begin{array}{c}t x+z^{3} y+z x^{4}+x^{2} y^{5} \subset \mathbb{P}(51,18,11,8) \\
\text { Diff }=\left(\frac{1}{2}, 0,0,0\right)+\frac{2}{3} \Upsilon_{4}\end{array}$ & 16 \\
\hline 127 & $\begin{array}{c}z^{3} y+z x^{4}+a x^{3} y^{3}+ \\
+t z y^{2}\end{array}$ & $\begin{array}{c}t^{2} x+g(z, x, y) \subset \mathbb{P}(16,11,7,6) \\
\text { Diff }=\frac{1}{2} \Delta_{3}\end{array}$ & 6 \\
\hline 128 & $z^{3} y+z x^{4}+z x y^{4}$ & $\begin{array}{c}t x+z^{3} y+z x^{4}+z x y^{4} \subset \mathbb{P}(37,13,8,6) \\
\text { Diff }=\frac{1}{2} \Gamma+\frac{3}{4} \Gamma_{2}\end{array}$ & 12 \\
\hline 129 & $z^{3} y+z x^{4}+t y^{4}$ & $\begin{array}{c}t^{2} x+z^{3} y+z x^{4}+t y^{4} \subset \mathbb{P}(39,27,17,14) \\
\text { Diff }=\frac{2}{3} \Upsilon_{4}\end{array}$ & 7 \\
\hline 130 & $\begin{array}{c}z^{3} y+z x^{3} y+y^{2 n} \\
n=3 ; 4\end{array}$ & $\begin{aligned} t^{2} x+g(z, x, y) & \subset \mathbb{P}(7 n+1,6 n-3,4 n-2,9) \\
& \operatorname{Diff}=\frac{2 n-2}{2 n-1} \Gamma_{4}\end{aligned}$ & 5,9 \\
\hline 131 & $\begin{array}{l}z^{3} y+z x^{3} y+a z y^{5}+ \\
+b x^{3} y^{3}+c y^{7}\end{array}$ & $\begin{array}{c}t x+g(z, x, y) \subset \mathbb{P}(17,6,4,3) \\
\text { Diff }=\frac{1}{2} \Gamma+\frac{3}{4} \Gamma_{4}\end{array}$ & 6 \\
\hline 132 & $z^{3} y+z x^{3} y+y^{9}$ & $\begin{array}{c}t x+g(z, x, y) \subset \mathbb{P}(65,24,16,9) \\
\text { Diff }=\frac{1}{2} \Gamma+\frac{15}{16} \Gamma_{4}\end{array}$ & 18 \\
\hline 133 & $\begin{array}{c}z^{3} y+z x^{3} y+x y^{n} \\
n=5 ; 7\end{array}$ & $\begin{array}{c}t x+g(z, x, y) \subset \mathbb{P}(7 n, 3 n-3,2 n-2,7) \\
\text { Diff }=\frac{1}{2} \Gamma+\frac{6}{7} \Delta_{3}+\frac{2 n-3}{2 n-2} \Gamma_{4}\end{array}$ & 8,14 \\
\hline 134 & $z^{3} y+z x^{3} y+x y^{6}$ & $\begin{array}{c}t^{2} x+z^{3} y+z x^{3} y+x y^{6} \subset \mathbb{P}(21,15,10,7) \\
\text { Diff }=\frac{6}{7} \Delta_{3}+\frac{4}{5} \Gamma_{4}+\frac{2}{3} \Upsilon_{4}\end{array}$ & 7 \\
\hline 135 & $\begin{array}{l}z^{3} y+z x^{3} y+a t z y^{2}+ \\
\quad+b x^{2} y^{4}, a \neq 0\end{array}$ & $\begin{array}{c}t^{2} x+g(z, x, y) \subset \mathbb{P}(13,9,6,5) \\
\text { Diff }=\frac{2}{3} \Gamma_{4}\end{array}$ & 5 \\
\hline 136 & $z^{3} y+z x^{3} y+x^{2} y^{5}$ & $\begin{array}{c}t x+z^{3} y+z x^{3} y+x^{2} y^{5} \subset \mathbb{P}(33,12,8,5) \\
\text { Diff }=\frac{1}{2} \Gamma+\frac{7}{8} \Gamma_{4}+\frac{2}{3} \Upsilon_{4}\end{array}$ & 10 \\
\hline 137 & $z^{3} y+z x^{3} y+z y^{6}$ & $\begin{array}{c}t x+z^{3} y+z x^{3} y+z y^{6} \subset \mathbb{P}(41,15,10,6) \\
\text { Diff }=\frac{1}{2} \Gamma+\frac{3}{4} \Gamma_{2}+\frac{9}{10} \Gamma_{4}\end{array}$ & 12 \\
\hline 138 & $z^{3} y+z x^{3} y+z x y^{4}$ & $\begin{array}{c}t x+z^{3} y+z x^{3} y+z x y^{4} \subset \mathbb{P}(25,9,6,4) \\
\text { Diff }=\frac{1}{2} \Gamma+\frac{3}{4} \Gamma_{2}+\frac{5}{6} \Gamma_{4}\end{array}$ & 8 \\
\hline 139 & $\begin{array}{c}z^{3} y+z x^{3} y+t y^{n} \\
n=4^{*} ; 5\end{array}$ & $\begin{array}{c}t^{2} x+g(z, x, y) \subset \mathbb{P}(7 n+2,6 n-3,4 n-2,11) \\
\text { Diff }=\frac{2 n-2}{2 n-1} \Gamma_{4}+\left(\frac{2}{3} \Upsilon_{4}\right)^{*}\end{array}$ & 7,11 \\
\hline 140 & $z^{3} y+z^{2} x^{2}+x y^{6}$ & $\begin{array}{c}t^{2} x+z^{3} y+z^{2} x^{2}+x y^{6} \subset \mathbb{P}(15,11,8,5) \\
\text { Diff }=\frac{4}{5} \Delta_{3}\end{array}$ & 5 \\
\hline 141 & $\begin{array}{c}z^{3} y+z^{2} x^{2}+x y^{n} \\
n=5 ; 7\end{array}$ & $\begin{array}{c}t x+g(z, x, y) \subset \mathbb{P}(5 n, 2 n-1, n+2,5) \\
\text { Diff }=\left(\frac{1}{2}, 0,0,0\right)+\frac{4}{5} \Delta_{3}\end{array}$ & 6,10 \\
\hline
\end{tabular}


9. Singularity $-t^{2} y+g(z, x, y)$.

\begin{tabular}{|c|c|c|c|}
\hline 1 & $\begin{array}{c}z^{4}+z x^{4}+a z^{2} x y^{2}+ \\
+b x^{2} y^{4}\end{array}$ & $\begin{array}{c}t y+g(z, x, y) \subset \mathbb{P}(27,8,6,5) \\
\text { Diff }=\left(\frac{1}{2}, 0,0,0\right)+\frac{2}{3} \Delta_{4}\end{array}$ & 10 \\
\hline 2 & $z^{4}+z x^{4}+z y^{5}$ & $\begin{array}{c}t^{2} y+z^{4}+z x^{2}+z y^{5} \subset \mathbb{P}(17,10,15,6) \\
\text { Diff }=\left(0,0, \frac{1}{2}, 0\right)+\frac{2}{3} \Gamma_{2}\end{array}$ & 12 \\
\hline 3 & $z^{4}+z x^{4}+z x y^{4}$ & $\begin{array}{c}t y+z^{4}+z x^{4}+z x y^{4} \subset \mathbb{P}(55,16,12,9) \\
\text { Diff }=\frac{1}{2} \Gamma+\frac{5}{6} \Gamma_{2}\end{array}$ & 18 \\
\hline 4 & $\begin{array}{c}z^{3} x+x^{5}+a z x^{2} y^{3}+ \\
+b y^{9}\end{array}$ & $\begin{array}{c}t^{2} y+g(z, x, y) \subset \mathbb{P}(20,12,9,5) \\
\text { Diff }=\frac{3}{4} \Upsilon_{4}\end{array}$ & 5 \\
\hline 5 & $z^{3} x+x^{5}+y^{13}$ & $\begin{array}{c}t^{2} y+z x+x^{5}+y^{13} \subset \mathbb{P}(30,52,13,5) \\
\text { Diff }=\left(0, \frac{2}{3}, 0,0\right)+\frac{1}{2} \Upsilon_{4}\end{array}$ & 15 \\
\hline 6 & $\begin{array}{l}z^{3} x+x^{5}+x y^{n} \\
n=5 ; 7 ; 11\end{array}$ & $\begin{array}{c}t y+z x+x^{5}+x y^{n} \subset \mathbb{P}(5 n-4,4 n, n, 4) \\
\text { Diff }=\left(0, \frac{2}{3}, 0,0\right)+\frac{1}{2} \Gamma+\frac{7}{8} \Gamma_{3}\end{array}$ & $9,16,24$ \\
\hline 7 & $\begin{array}{c}z^{3} x+x^{5}+a z x^{3} y+ \\
b z x y^{4}+c x^{3} y^{3}+d x y^{6} \\
|b|+|d| \neq 0\end{array}$ & $\begin{array}{c}t y+g(z, x, y) \subset \mathbb{P}(13,4,3,2) \\
\text { Diff }=\frac{1}{2} \Gamma+\frac{3}{4} \Gamma_{3}\end{array}$ & 4 \\
\hline 8 & $\begin{aligned} z^{3} x+ & x^{5}+a z x y^{6}+ \\
& +b x y^{9}\end{aligned}$ & $\begin{array}{c}t y+g(z, x, y) \subset \mathbb{P}(41,12,9,4) \\
\text { Diff }=\frac{1}{2} \Gamma+\frac{7}{8} \Gamma_{3}\end{array}$ & 8 \\
\hline 9 & $\begin{aligned} z^{3} x+ & x^{5}+a x^{3} y^{5}+ \\
& +b x y^{10}\end{aligned}$ & $\begin{array}{c}t y+g\left(z^{1 / 3}, x, y\right) \subset \mathbb{P}(23,20,5,2) \\
\text { Diff }=\left(0, \frac{2}{3}, 0,0\right)+\frac{1}{2} \Gamma+\frac{3}{4} \Gamma_{3}\end{array}$ & 12 \\
\hline 10 & $z^{3} x+x^{5}+x^{2} y^{7}$ & $\begin{array}{c}t^{2} y+z x+x^{5}+x^{2} y^{7} \subset \mathbb{P}(16,28,7,3) \\
\text { Diff }=\left(0, \frac{2}{3}, 0,0\right)+\frac{3}{4} \Upsilon_{4}\end{array}$ & 9 \\
\hline 11 & $\begin{array}{c}z^{3} x+x^{5}+z y^{2 n+1} \\
n=2 ; 4^{*}\end{array}$ & $\begin{array}{c}t^{2} y+g(z, x, y) \subset \mathbb{P}(15 n+2,8 n+4,6 n+3,11) \\
\text { Diff }=\frac{3}{4} \Upsilon_{4} ;\left(\frac{1}{2} \Upsilon_{4}\right)^{*}\end{array}$ & 5,11 \\
\hline 12 & $\begin{array}{c}z^{3} x+x^{5}+z x y^{n} \\
n=3 ; 5 ; 7\end{array}$ & $\begin{array}{c}t y+g(z, x, y) \subset \mathbb{P}(15 n-8,4 n, 3 n, 8) \\
\text { Diff }=\frac{1}{2} \Gamma+\frac{7}{8} \Gamma_{3}\end{array}$ & $8,10,16$ \\
\hline 13 & $z^{3} x+x^{5}+z^{2} y^{5}$ & $\begin{array}{c}t^{2} y+z^{3} x+x^{5}+z^{2} y^{5} \subset \mathbb{P}(34,20,15,7) \\
\text { Diff }=\frac{1}{2} \Upsilon_{4}\end{array}$ & 7 \\
\hline 14 & $\begin{aligned} z^{3} x+ & x^{6}+a z x y^{4}+ \\
& +b x y^{6}\end{aligned}$ & $\begin{array}{c}t y+g(z, x, y) \subset \mathbb{P}(31,10,6,5) \\
\text { Diff }=\frac{1}{2} \Gamma+\frac{9}{10} \Gamma_{3}\end{array}$ & 10 \\
\hline 15 & $z^{3} x+x^{6}+x y^{7}$ & $\begin{array}{c}t y+z x+x^{6}+x y^{7} \subset \mathbb{P}(37,35,7,5) \\
\text { Diff }=\left(0, \frac{2}{3}, 0,0\right)+\frac{1}{2} \Gamma+\frac{9}{10} \Gamma_{3}\end{array}$ & 30 \\
\hline 16 & $\begin{array}{c}z^{3} x+x^{5} y+y^{2 n+1} \\
n=3 ; 4\end{array}$ & $\begin{array}{c}t^{2} y+g\left(z^{1 / 3}, x, y\right) \subset \mathbb{P}(5 n, 8 n+5,2 n, 5) \\
\text { Diff }=\frac{2}{3} \Delta+\frac{3 n-1}{3 n} \Delta_{4}\end{array}$ & 9,15 \\
\hline 17 & $\begin{array}{c}z^{3} x+x^{5} y+a z x^{2} y^{3}+ \\
+b y^{8}\end{array}$ & $\begin{array}{c}t y+g(z, x, y) \subset \mathbb{P}(35,11,7,5) \\
\text { Diff }=\left(\frac{1}{2}, 0,0,0\right)+\frac{6}{7} \Delta_{4}\end{array}$ & 10 \\
\hline 18 & $z^{3} x+x^{5} y+y^{10}$ & $\begin{array}{c}t y+z x+x^{5} y+y^{10} \subset \mathbb{P}(45,41,9,5) \\
\text { Diff }=\left(\frac{1}{2}, 0,0,0\right)+\frac{2}{3} \Delta+\frac{26}{27} \Delta_{4}\end{array}$ & 30 \\
\hline 19 & $\begin{array}{c}z^{3} x+x^{5} y+a z x y^{4}+ \\
+b x y^{6}\end{array}$ & $\begin{array}{c}t y+g(z, x, y) \subset \mathbb{P}(25,8,5,4) \\
\text { Diff }=\frac{1}{2} \Gamma+\frac{7}{8} \Gamma_{3}+\frac{4}{5} \Delta_{4}\end{array}$ & 8 \\
\hline 20 & $\begin{array}{c}z^{3} x+x^{5} y+a x^{3} y^{4}+ \\
+b x y^{7}\end{array}$ & $\begin{array}{c}t y+g\left(z^{1 / 3}, x, y\right) \subset \mathbb{P}(15,14,3,2) \\
\text { Diff }=\frac{1}{2} \Gamma+\frac{2}{3} \Delta+\frac{3}{4} \Gamma_{3}+\frac{8}{9} \Delta_{4}\end{array}$ & 12 \\
\hline
\end{tabular}




\begin{tabular}{|c|c|c|c|}
\hline 21 & $z^{3} x+x^{5} y+x y^{8}$ & $\begin{array}{c}t y+z x+x^{5} y+x y^{8} \subset \mathbb{P}(35,32,7,4) \\
\text { Diff }=\frac{1}{2} \Gamma+\frac{2}{3} \Delta+\frac{7}{8} \Gamma_{3}+\frac{20}{21} \Delta_{4}\end{array}$ & 24 \\
\hline 22 & $z^{3} x+x^{5} y+x^{2} y^{5}$ & $\begin{array}{c}t^{2} y+z x+x^{5} y+x^{2} y^{5} \subset \mathbb{P}(10,19,4,3) \\
\text { Diff }=\frac{2}{3} \Delta+\frac{5}{6} \Delta_{4}\end{array}$ & 9 \\
\hline 23 & $z^{3} x+x^{5} y+x^{2} y^{6}$ & $\begin{array}{c}t y+z x+x^{5} y+x^{2} y^{6} \subset \mathbb{P}(25,23,5,3) \\
\text { Diff }=\left(\frac{1}{2}, 0,0,0\right)+\frac{2}{3} \Delta+\frac{14}{15} \Delta_{4}\end{array}$ & 18 \\
\hline 24 & $\begin{array}{l}z^{3} x+x^{5} y+z y^{n} \\
n=5 ; 7\end{array}$ & $\begin{array}{c}t y+g(z, x, y) \subset \mathbb{P}(15 n-10,4 n+1,3 n-2,11) \\
\text { Diff }=\left(\frac{1}{2}, 0,0,0\right)+\frac{3 n-3}{3 n-2} \Delta_{4}\end{array}$ & 14,22 \\
\hline 25 & $z^{3} x+x^{5} y+z y^{6}$ & $\begin{array}{c}t^{2} y+z^{3} x+x^{5} y+z y^{6} \subset \mathbb{P}(40,25,16,11) \\
\text { Diff }=\frac{4}{5} \Upsilon_{4}+\frac{7}{8} \Delta_{4}\end{array}$ & 11 \\
\hline 26 & $z^{3} x+x^{5} y+z x y^{5}$ & $\begin{array}{c}t y+z^{3} x+x^{5} y+z x y^{5} \subset \mathbb{P}(65,20,13,8) \\
\text { Diff }=\frac{1}{2} \Gamma+\frac{7}{8} \Delta_{3}+\frac{4}{5} \Upsilon_{4}\end{array}$ & 16 \\
\hline 27 & $z^{3} x+x^{5} y+z^{2} y^{3}$ & $\begin{array}{c}t^{2} y+z^{3} x+x^{5} y+z^{2} y^{3} \subset \mathbb{P}(20,13,8,7) \\
\text { Diff }=\frac{3}{4} \Delta_{4}\end{array}$ & 7 \\
\hline 28 & $z^{3} x+x^{5} y+z^{2} y^{4}$ & $\begin{array}{c}t y+z^{3} x+x^{5} y+z^{2} y^{4} \subset \mathbb{P}(55,17,11,7) \\
\text { Diff }=\left(\frac{1}{2}, 0,0,0\right)+\frac{10}{11} \Delta_{4}\end{array}$ & 14 \\
\hline 27 & $z^{3} x+z x^{4}+y^{7}$ & $\begin{array}{c}t^{2} y+z^{3} x+z x^{4}+y^{7} \subset \mathbb{P}(33,21,14,11) \\
\text { Diff }=\frac{2}{3} \Upsilon_{4}\end{array}$ & 7 \\
\hline 28 & $z^{3} x+z x^{4}+y^{9}$ & $\begin{array}{c}t^{2} y+z^{3} x+z x^{4}+y^{9} \subset \mathbb{P}(44,27,18,11) \\
\text { Diff }=\frac{1}{2} \Delta_{4}\end{array}$ & 11 \\
\hline 29 & $z^{3} x+z x^{4}+y^{10}$ & $\begin{array}{c}t y+z^{3} x+z x^{4}+y^{10} \subset \mathbb{P}(99,30,20,11) \\
\text { Diff }=\left(\frac{1}{2}, 0,0,0\right)+\frac{2}{3} \Upsilon_{4}\end{array}$ & 22 \\
\hline 30 & $\begin{array}{c}z^{3} x+z x^{4}+x y^{2 n+1} \\
n=2 ; 3\end{array}$ & $\begin{array}{c}t^{2} y+g(z, x, y) \subset \mathbb{P}(11 n+1,6 n+3,4 n+2,9) \\
\operatorname{Diff}=\frac{2}{3} \Gamma_{3}+\frac{n-2}{n-1} \Delta_{4}\end{array}$ & 5,9 \\
\hline 31 & $\begin{array}{l}z^{3} x+z x^{4}+a z x y^{4}+ \\
\quad+b x^{4} y^{2}+c x y^{6}\end{array}$ & $\begin{array}{c}t y+g(z, x, y) \subset \mathbb{P}(19,6,4,3) \\
\text { Diff }=\frac{1}{2} \Gamma+\frac{5}{6} \Gamma_{3}\end{array}$ & 6 \\
\hline 32 & $z^{3} x+z x^{4}+x y^{8}$ & $\begin{array}{c}t y+z^{3} x+z x^{4}+x y^{8} \subset \mathbb{P}(79,24,16,9) \\
\text { Diff }=\frac{1}{2} \Gamma+\frac{5}{6} \Gamma_{3}\end{array}$ & 18 \\
\hline 33 & $z^{3} x+z x^{4}+x^{2} y^{5}$ & $\begin{array}{c}t^{2} y+z^{3} x+z x^{4}+x^{2} y^{5} \subset \mathbb{P}(24,15,10,7) \\
\text { Diff }=\frac{1}{2} \Delta_{4}\end{array}$ & 7 \\
\hline 34 & $\begin{array}{l}z^{3} x+z x^{4}+a z^{2} y^{3}+ \\
\quad+b x^{3} y^{3}, a \neq 0\end{array}$ & $\begin{array}{c}t^{2} y+g(z, x, y) \subset \mathbb{P}(14,9,6,5) \\
\text { Diff }=\frac{1}{2} \Delta_{4}\end{array}$ & 5 \\
\hline 35 & $\begin{aligned} z^{3} x+ & z x^{4}+a z^{2} y^{4}+ \\
& +b x^{3} y^{4}\end{aligned}$ & $\begin{array}{c}t y+g(z, x, y) \subset \mathbb{P}(39,12,8,5) \\
\operatorname{Diff}=\left(\frac{1}{2}, 0,0,0\right)+\frac{2}{3} \Upsilon_{4}\end{array}$ & 10 \\
\hline 36 & $\begin{array}{c}z^{3} x+z x^{4}+z y^{n} \\
n=5 ; 7\end{array}$ & $\begin{array}{c}t y+g(z, x, y) \subset \mathbb{P}(11 n-8,3 n, 2 n, 8) \\
\text { Diff }=\frac{1}{2} \Gamma+\frac{3}{4} \Gamma_{2}+\frac{n-5}{n-4} \Upsilon_{4}\end{array}$ & 10,16 \\
\hline 37 & $\begin{array}{c}z^{3} x+z x^{4}+a z x^{2} y^{3}+ \\
+b z y^{6}\end{array}$ & $\begin{array}{c}t y+g(z, x, y) \subset \mathbb{P}(29,9,6,4) \\
\text { Diff }=\frac{1}{2} \Gamma+\frac{3}{4} \Gamma_{2}\end{array}$ & 8 \\
\hline 38 & $z^{3} x+z x^{4}+z x y^{5}$ & $\begin{array}{c}t y+z^{3} x+z x^{4}+z x y^{5} \subset \mathbb{P}(49,15,10,6) \\
\text { Diff }=\frac{1}{2} \Gamma+\frac{3}{4} \Gamma_{2}+\frac{5}{6} \Gamma_{3}\end{array}$ & 12 \\
\hline 39 & $\begin{array}{c}z^{3} x+t x^{3}+a x^{3} y^{4}+ \\
+b y^{9}\end{array}$ & $\begin{array}{c}t^{2} y+g\left(z^{1 / 3}, x, y\right) \subset \mathbb{P}(12,22,5,3) \\
\text { Diff }=\left(0, \frac{2}{3}, 0,0\right)+\frac{1}{2} \Upsilon_{4}\end{array}$ & 9 \\
\hline 40 & $\begin{array}{c}z^{3} x+t x^{3}+x y^{n} \\
n=5 ; 7\end{array}$ & $\begin{array}{c}t^{2} y+g\left(z^{1 / 3}, x, y\right) \subset \mathbb{P}(3 n-2,5 n, n+1,5) \\
\text { Diff }=\left(0, \frac{2}{3}, 0,0\right)+\frac{4}{5} \Gamma_{3}\end{array}$ & 9,12 \\
\hline
\end{tabular}




\begin{tabular}{|c|c|c|c|}
\hline 41 & $\begin{array}{c}z^{3} x+t x^{3}+a z x y^{4}+ \\
+b x y^{6}\end{array}$ & $t^{2} y+g(z, x, y) \subset \mathbb{P}(16,10,7,5)$ & 5 \\
& & Diff $=\frac{4}{5} \Gamma_{3}+\frac{1}{2} \Upsilon_{4}$ & \\
\hline 42 & $z^{3} x+t x^{3}+x y^{8}$ & $t^{2} y+z x+t x^{3}+x y^{8} \subset \mathbb{P}(22,40,9,5)$ & 15 \\
& & Diff $=\left(0, \frac{2}{3}, 0,0\right)+\frac{4}{5} \Gamma_{3}+\frac{1}{2} \Upsilon_{4}$ & \\
\hline 43 & $z^{3} x+t x^{3}+z y^{2 n+1}$ & $t^{2} y+g(z, x, y) \subset \mathbb{P}(18 n+2,10 n+4,6 n+5,13)$ & 8,13 \\
& $n=2 ; 3$ & Diff $=\frac{1}{2} \Upsilon_{4}$ & \\
\hline 44 & $z^{3} x+t x^{3}+z x y^{n}$ & $t^{2} y+g(z, x, y) \subset \mathbb{P}(9 n-4,5 n, 3 n+2,10)$ & 5,10 \\
& $n=3 ; 5$ & Diff $=\frac{4}{5} \Gamma_{3}$ & \\
\hline 45 & $z^{3} x+t x^{3}+z x^{2} y^{3}$ & $t^{2} y+z^{3} x+t x^{3}+z x^{2} y^{3} \subset \mathbb{P}(26,16,11,7)$ & 7 \\
& & Diff $=\frac{1}{2} \Upsilon_{4}$ & \\
\hline
\end{tabular}




\section{REFERENCES}

[1] Iano-Fletcher A. R. Working with weighted complete intersections, in "Explicit birational geometry of 3-folds", 73-173, London Math. Soc. Lecture Note Ser., 281, Cambridge Univ. Press, Cambridge, 2000.

[2] Ishii $S$. The quotient of $\log$-canonical singularities by finite groups, Advanced Studies in Pure Math. 29, (2000) 135-161

[3] Kudryavtsev S. A. On purely log terminal blow-ups, Math. Notes 69 No. 6 (2001) e-print math.AG/0111278.

[4] Kudryavtsev S. A. Classification of three-dimensional exceptional log canonical hypersurface singularities I, appear in Russian Acad. Sci. Izv. Math. (2002) eprint math.AG/0201025.

[5] Prokhorov Yu. G. Blow-ups of canonical singularities, Algebra. Proc. Internat. Conf. on the Occasion of the 90th birthday of A. G. Kurosh, Moscow, Russia, May 25-30, 1998, Yu. Bahturin ed., Walter de Gruyter, Berlin (2000), 301-317

[6] Shokurov V. V. 3-fold log flips, Russian Acad. Sci. Izv. Math. 40 (1993) 93-202 \& 43 (1994) 527-558

Department of Algebra, Faculty of Mathematics, Moscow State Lomonosov University, 117234 Moscow, Russia

E-mail address: kudryav@mech.math.msu.su 